\title{
VOLTAGE CONTROLLED OSCILLATOR PHASE NOISE REDUCTION TECHNIQUE
}

By

Céline E.M. Fletcher

\author{
A thesis \\ presented to Carleton University \\ in fulfilment of the \\ thesis requirement for the degree of \\ MASTER OF APPLIED SCIENCE \\ in \\ ELECTRICAL ENGINEERING
}

Ottawa, Ontario, Canada

(C) Céline E.M. Fletcher, 2006 


$\begin{array}{ll}\begin{array}{l}\text { Library and } \\ \text { Archives Canada }\end{array} & \begin{array}{l}\text { Bibliothèque et } \\ \text { Archives Canada }\end{array} \\ \begin{array}{l}\text { Published Heritage } \\ \text { Branch }\end{array} & \begin{array}{l}\text { Direction du } \\ \text { Patrimoine de l'édition }\end{array} \\ \begin{array}{l}\text { 395 Wellington Street } \\ \text { Ottawa ON K1A ON4 }\end{array} & \begin{array}{l}\text { 395, rue Wellington } \\ \text { Ottawa ON K1A ON4 } \\ \text { Canada }\end{array}\end{array}$

Your file Votre référence ISBN: 978-0-494-16456-3 Our file Notre référence ISBN: 978-0-494-16456-3

NOTICE:

The author has granted a nonexclusive license allowing Library and Archives Canada to reproduce, publish, archive, preserve, conserve, communicate to the public by telecommunication or on the Internet, loan, distribute and sell theses worldwide, for commercial or noncommercial purposes, in microform, paper, electronic and/or any other formats.

The author retains copyright ownership and moral rights in this thesis. Neither the thesis nor substantial extracts from it may be printed or otherwise reproduced without the author's permission.
AVIS:

L'auteur a accordé une licence non exclusive permettant à la Bibliothèque et Archives Canada de reproduire, publier, archiver, sauvegarder, conserver, transmettre au public par télécommunication ou par l'Internet, prêter, distribuer et vendre des thèses partout dans le monde, à des fins commerciales ou autres, sur support microforme, papier, électronique et/ou autres formats.

L'auteur conserve la propriété du droit d'auteur et des droits moraux qui protège cette thèse. $\mathrm{Ni}$ la thèse ni des extraits substantiels de celle-ci ne doivent être imprimés ou autrement reproduits sans son autorisation.
In compliance with the Canadian

Privacy Act some supporting forms may have been removed from this thesis.

While these forms may be included in the document page count, their removal does not represent any loss of content from the thesis.
Conformément à la loi canadienne sur la protection de la vie privée, quelques formulaires secondaires ont été enlevés de cette thèse.

Bien que ces formulaires aient inclus dans la pagination, il n'y aura aucun contenu manquant.

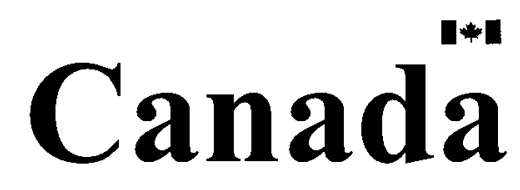




\begin{abstract}
A phase noise reduction technique using a switched-bias circuit is presented. The switchedbias circuit is integrated into the bias network of a voltage controlled oscillator (VCO) and is implemented in $0.18 \mu \mathrm{m}$ CMOS technology. The VCO is an LC oscillator with a center frequency of $1.4 \mathrm{GHz}$. A review of noise sources in CMOS and state-of-the-art phase noise reductions techniques is discussed. The design of the switched-bias circuit, consisting of an NMOS switch and a 10pF MIM capacitor, and the integration of the circuit into the LC VCO is presented. For the exemplary VCO and switched-bias circuit presented in this work, simulations have shown that the VCO phase noise was reduced by $6 \mathrm{~dB}$, for frequency offsets between $100 \mathrm{kHz}$ and $1 \mathrm{MHz}$.
\end{abstract}




\section{Acknowledgements}

The financial support provided by the National Sciences and Engineering Research Council of Canada (NSERC) and Carleton University has helped make this research possible. Thank you also to CMC Microsystems who provided access to the TSMC fabrication facilities.

Special thanks is given to Vladimir Vukovic for his work on the PCB layout, Laurent Mouden at École Polytechnique in Montréal for his expertise and assistance in wire bonding the fabricated chips, and Nagui Mikhail for his technical advice and keeping our lab running.

I would also like to thank several of my fellow graduate students, in particular, Fiona Shearer, Daniel Olszewski, Mark Houlgate, Sinisa Milicevic, John Danson, Jeff Slater and Yasser Soliman who have helped provide me with a great working environment. In addition, I would like to thank Jennifer Sander and Sabina Choudhury for being great friends and for always being there to listen.

And most importantly I'd like to thank my parents, Colette and Joseph Fletcher, my

sister, Émilie Fletcher and my husband, John Phillips, for their love and encouragement, which have made this work possible. 
to Mom, Dad, Émilie and John 


\section{Table of Contents}

$\begin{array}{ll}\text { Abstract } & \text { ii }\end{array}$

Acknowledgements

Table of Contents $\quad$ v

List of Tables viii

List of Figures $\quad$ ix

List of Abbreviations $\quad$ xii

1 Introduction 1

1.1 Motivation . . . . . . . . . . . . . . . . . . . . 1

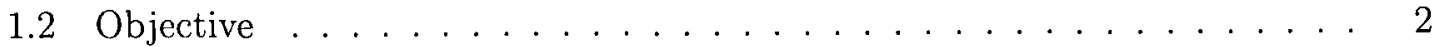

1.3 Thesis Organization . . . . . . . . . . . . . . . 2

2 Background Information 3

2.1 System Level Requirements for Phase Noise . . . . . . . . . . . . . . . . . . 3

2.2 Techniques Used to Reduce Phase Noise in VCOs . . . . . . . . . . . . 6

2.2.1 Elimination of the Bias Circuit in a VCO . . . . . . . . . . 6

2.2 .2 Filtering Noise . . . . . . . . . . . . . . . . . . 9

2.2 .3 Using Topology to reduce noise . . . . . . . . . . . . 11

2.3 CMOS Intrinsic Noise Sources . . . . . . . . . . . . . . . . . 18

2.3 .1 Thermal Noise . . . . . . . . . . . . . . . . 18

2.3 .2 Flicker Noise . . . . . . . . . . . . . . . . . . . . 19

2.4 CMOS Extrinsic Noise Sources _. . . . . . . . . . . . . . . . . 19

2.4 Power Supply Noise . . . . . . . . . . . . . . . . . . . . . 20

2.4 .2 Substrate Noise . . . . . . . . . . . . . . . . . 21

2.4 .3 Bias Noise . . . . . . . . . . . . . . . . . . 22

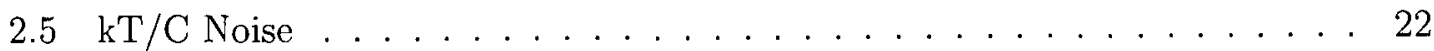

2.6 Phase Noise . . . . . . . . . . . . . . . . . . . . . . . . . . . 24 
2.6.1 Leeson's Equation . . . . . . . . . . . . . . . . . . . . . . . 24

2.7 Chapter Summary . . . . . . . . . . . . . . 26

3 Switched-Bias Method and Implementation 27

3.1 Switched-Bias Method . . . . . . . . . . . . . . . 27

3.1.1 Overview of the Switched-Bias Method . . . . . . . . . . . 27

3.2 Implementation . . . . . . . . . . . . . . . . . . . . . 28

3.2 .1 Switched-bias circuit . . . . . . . . . . . . . . . . 28

3.2 .2 Switch and Capacitor Sizing . . . . . . . . . . . . . . . . . 30

3.3 Design Issues . . . . . . . . . . . . . . . . . . . . . . 35

3.3.1 Voltage Variation at Bias Node . . . . . . . . . . . . . . . 35

3.3 .2 Sizing of Current Mirror . . . . . . . . . . . . . . . . . . . 38

3.4 Application Analysis . . . . . . . . . . . . . . . . . . . 39

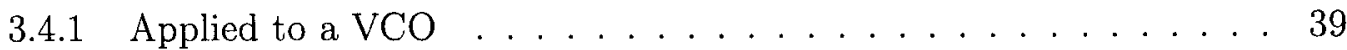

3.4 Analysis . . . . . . . . . . . . . . . . . . 39

3.4 .3 Noise Contribution Simulation . . . . . . . . . . . . . . . . . 43

3.4 .4 Phase Noise Simulations . . . . . . . . . . . . . . . . . . 47

3.5 Chapter Summary . . . . . . . . . . . . . . . . . . . . . . . 48

4 Design and Imlpementation $\quad 49$

4.1 VCO design . . . . . . . . . . . . . . . . . . . 49

4.2 Integration of switched-bias circuit into the $\mathrm{VCO} \ldots \ldots \ldots 53$

4.3 Layout Considerations . . . . . . . . . . . . . . . . . . 56

4.4 Cadence Simulations . . . . . . . . . . . . . . . . . . . . . 61

4.5 ADS Simulations . . . . . . . . . . . . . . . . . . 64

4.6 Impact of spurs on radio system . . . . . . . . . . . . . 70

4.7 Analysis of Switching Noise . . . . . . . . . . . . . . . . . . . 72

4.7.1 Transient Noise Simulation . . . . . . . . . . . . . . . . 80

4.7 .2 Spot Noise . . . . . . . . . . . . . . . . . 86

4.8 Spur Simulations . . . . . . . . . . . . . . . . . . 88

4.9 Flicker Noise: Slope of Phase Noise Plot . . . . . . . . . . . . . . 93

4.10 Chapter Summary . . . . . . . . . . . . . . . . . 94

5 Measurement Results $\quad 95$

5.1 PCB design . . . . . . . . . . . . . . . . . . . . . . . . . . . . . . . . .

5.1 .1 Power Supply and DC inputs . . . . . . . . . . . . . 99

5.1 .2 Current Sources . . . . . . . . . . . . . . . . . . . . 99

5.1 .3 ESD protection . . . . . . . . . . . . . . . . . 101

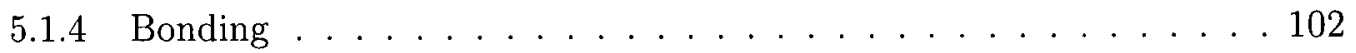

5.1 .5 RF output . . . . . . . . . . . . . . . . . . . 104

5.2 Test setup and Measurement Results . . . . . . . . . . . . . . . . . 104

5.2 .1 Test Equipment . . . . . . . . . . . . . . . . . . . . 104 
5.2 .2 Testing of VCO without Switched-Bias Circuit . . . . . . . . . . . 105

5.2.3 Testing of the VCO with the switched-bias circuit . . . . . . . 110

5.2.4 Challenges encountered during the testing of the VCO with the switched-bias circuit . . . . . . . . . . . . . . 113

5.3 Chapter Summary . . . . . . . . . . . . . . . . . . 119

6 Conclusion $\quad \mathbf{1 2 0}$

6.1 Summary of the results obtained . . . . . . . . . . . . . . 120

6.2 Contribution to Research . . . . . . . . . . . . . . . . . . 120

6.3 Future Work . . . . . . . . . . . . . . . . . . . . . . 121

$\begin{array}{ll}\text { Appendix A PCB Components } & 123\end{array}$

$\begin{array}{ll}\text { Bibliography } & 124\end{array}$ 


\section{List of Tables}

2.1 Comparison of LC oscillator phase noise performance . . . . . . . . . 4

2.2 Comparison of LC oscillator topologies used to reduce phase noise . . . . . 12

3.1 Transfer function small signal parameter values . . . . . . . . . . . . . . 41

3.2 PSS Simulation Parameters . . . . . . . . . . . . . . . . . . . . 43

3.3 Pnoise Simulation Parameters . . . . . . . . . . . . . . . . . 44

3.4 Phase noise for various states of the switch and offset frequencies . . . . . 47

4.1 Summary of simulation results for the cross-coupled LC VCO . . . . . . 53

4.2 Phase Noise Measurements . . . . . . . . . . . . . . . . . . . . . 53

4.3 List of the pad names used for the placement of the VCO . . . . . . . . . 58

4.4 Harmonic Balance Simulation Parameters . . . . . . . . . . . . . . . 65

4.5 Summary of parameters used to evaluate the noise due to the switched bias circuit . . . . . . . . . . . . . . . . 87

5.1 List of the signals connected to the SMA connectors . . . . . . . . . . . . 97

5.2 Potentiometer resistance value and currents generated on the PCB . . . . . 101

5.3 Comparison of simulated and measured results for the VCO without the switched-bias circuit . . . . . . . . . . . . . . 105

5.4 Frequency values used to determine the tuning range of the VCO . . . . 109

A.1 Components used to populate the PCB that was described in section 5.1

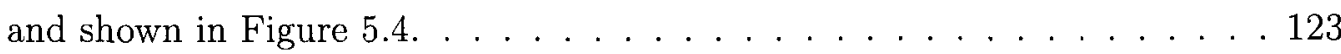




\section{List of Figures}

2.1 Relationship between BER and phase noise . . . . . . . . . 5

2.2 Schematic of a VCO containing a bias network to generate a tail current . 7

2.3 Schematic of a VCO without a bias network . . . . . . . . . . . 7

2.4 Top-bias VCO noise filtering technique . . . . . . . . . . . . . . . 10

2.5 Measurement results for noise filtering technique . . . . . . . . . . . 11

2.6 Low-power, low-phase noise VCO . . . . . . . . . . . . . . . . . . 13

2.7 Quadrature VCO schematic . . . . . . . . . . . . . . . . 14

2.8 LC CMOS VCO . . . . . . . . . . . . . . . . . . . . . 16

2.9 LC VCO with negative resistance . . . . . . . . . . . . . . 17

2.10 Intrinsic noise sources for a MOSFET . . . . . . . . . . . . . . . 18

2.11 Diagram showing supply voltages on a mixed-signal chip . . . . . . . . 20

2.12 Model of $\mathrm{kT} / \mathrm{C}$ noise in a circuit. . . . . . . . . . . . . . 23

2.13 Illustration of the phase noise versus frequency relationship . . . . . . . . 25

3.1 Diagram illustrating the circuit segmenting method . . . . . . . . . . . 28

3.2 Switching and bias retention elements . . . . . . . . . . . . . . . 30

3.3 Test setup determine the capacitor and switch sizes . . . . . . . . . . . . 32

3.4 Phase noise as switch width is swept . . . . . . . . . . . 33

3.5 Phase noise as capacitor size is swept $(100 \mathrm{kHz}$ offset $) \ldots \ldots$. . . . . . . 34

3.6 Phase noise as capacitor size is swept $(600 \mathrm{kHz}$ offset $) \ldots \ldots$. . . . . . 35

3.7 Phase noise as capacitor size is swept $(1 \mathrm{MHz}$ offset $) \ldots \ldots$. . . . . . . 36

3.8 Test setup used to model the effect of voltage variation . . . . . . . . . 37

3.9 Plot of frequency versus bias voltage . . . . . . . . . . . . . . . 38

3.10 Current mirror schematic . . . . . . . . . . . . . . . . . . . . . . 39

3.11 Transfer function small signal current mirror schematic . . . . . . . . . . . 40 
3.12 Testbench used to simulated the transfer function derived . . . . . . . . . 41

3.13 Simulated and calculated values for the transfer function derived . . . . . . 42

3.14 VCO circuit with the switched-bias circuitry . . . . . . . . . . . . . . . 44

3.15 Graph of MOSFET noise contributions . . . . . . . . . . . . . . 45

3.16 Phase noise simulations over various offset frequencies . . . . . . . . . . 48

4.1 VCO circuit only . . . . . . . . . . . . . . . . . 50

4.2 Model used to simulate the inductor. . . . . . . . . . . . . 52

4.3 The measured and simulated phase noise results for the VCO. . . . . . . 54

4.4 Biasing network . . . . . . . . . . . . . . . 55

4.5 Switched-bias circuit including circuitry added to facilitate testing. . . . . 55

4.6 Placement of the VCO circuit including the switched-bias circuitry. . . . . 57

4.7 A photo of the chip layout. . . . . . . . . . . . . . . . . . . 60

4.8 A photo of the layout for all three designs included on the fabricated chip. 62

4.9 Testbench for the ADS phase noise simulation. . . . . . . . . . . . 66

4.10 Comparison of phase noise simulations $\ldots \ldots \ldots \ldots 7$

4.11 SSB Phase noise simulations over various offset frequencies in ADS . . . 68

4.12 Decrease in phase noise as the switch goes from the ON state to the OFF state . . . . . . . . . . . . . . . . . . . . . 6 69

4.13 Phase noise difference for VCO with the switch ON and without a switch . 69

4.14 Impact of spurs on VCO frequency . . . . . . . . . . . . . . . 71

4.15 View of sampled flicker noise . . . . . . . . . . . . . 73

4.16 Track and hold circuit. . . . . . . . . . . . . . . . . . 78

4.17 Testbench for transient noise measurement . . . . . . . . . . . . . . 81

4.18 Spectrum of bias current . . . . . . . . . . . . . . . . . 82

4.19 Spectrum of bias current with noise . . . . . . . . . . . . . 83

4.20 Testbench for transient simulation for VCO with no switch . . . . . . . . . 84

4.21 VCO output spectrum with and without switch . . . . . . . . . 85

4.22 Plot of spot flicker and $\mathrm{kT} / \mathrm{C}$ noise . . . . . . . . . . . . . 87

4.23 Simulated and Theoretical PWL function for Vgs of M7 . . . . . . . . . 89

4.24 Testbench used to simulate effect of spurs . . . . . . . . . . . . . 90

4.25 Plot of spurs from the piecewise linear model simulaton . . . . . . . . . . . 92 
4.26 Slope of phase noise plot . . . . . . . . . . . . . . . . 94

5.1 The top view of the PCB layout. . . . . . . . . . . . 96

5.2 The bottom view of the PCB layout. . . . . . . . . . . . . 97

5.3 Block diagram showing the PCB layout. . . . . . . . . . . . . 98

5.4 Photo of the populated PCB. . . . . . . . . . . . . . 98

5.5 DC signal decoupling network. . . . . . . . . . . . . . . . 99

5.6 Current reference configurations . . . . . . . . . . . . . 100

5.7 ESD protection circuitry . . . . . . . . . . . . . . . . 102

5.8 Bonding diagram used to indicate bond wire connections between the chip and the PCB. . . . . . . . . . . . . . . . . . . 103

5.9 Photo of the chip bonded to the PCB. . . . . . . . . . . . . . . 104

5.10 Test setup for the VCO without the switched-bias circuit . . . . . . . . . 106

5.11 Oscilloscope VCO output plot . . . . . . . . . . . . . . . . . 106

5.12 Spectrum Analyzer VCO output plot . . . . . . . . . . . . . . 107

5.13 VCO simulated and measured phase noise values . . . . . . . . . . . 108

5.14 Test setup for the VCO with the switched-bias circuit . . . . . . . . . . 110

5.15 Voltage change of the bias retention element . . . . . . . . . . . 111

5.16 Voltage variation, $\Delta V_{B}$ at the bias node versus switch clock frequency . . . 111

5.17 Oscilloscope probe leakage . . . . . . . . . . . . . . . 112

5.18 VCO buffer measurement results . . . . . . . . . . . . . 115

5.19 Testbench for bond wire length simulation . . . . . . . . . . . 116

5.20 Bond wire length versus VCO output frequency . . . . . . . . . . . . 117

5.21 Temperature versus VCO output frequency . . . . . . . . . . . . . . . 118 


\section{List of Abbreviations}

BER bit error rate

BSS base station system

Cgd gate-drain capacitance

Cgs gate-source capacitance

CMC CMC Microsystems

CMOS complementary metal oxide semiconductor

dB decibel

$\mathrm{dBm}$ decibel reference to $1 \mathrm{~mW}$

ESD electrostatic discharge

F noise factor

f frequency

gm transconductance

GND ground connection

GSM global system for mobile communication

MIM metal-insulator-metal

MOSCAP capacitor formed from a MOSFET by connecting the drain and source of the MOSFET together 
NMOS N-type metal oxide semiconductor

OSS operation and support system

PDF power density function

Q quality factor of the tank in the VCO

$\mathrm{RF}$ radio frequency

SNR signal to noise

SS switching system

SSB single-sideband

TDMA time division multiple access

TSMC Taiwan Semiconductor Manufacturing Company Limited

VCO voltage controlled oscillator

Vctrl Control voltage used to tune the VCO

VDD Power supply voltage 


\section{Chapter 1}

\section{Introduction}

This thesis discusses a circuit that has been implemented to reduce the phase noise in a voltage controlled oscillator.

\subsection{Motivation}

There is active interest in understanding and exploring ways to reduce phase noise in VCOs, especially in RF applications, as can be seen in [1-7]. It would be advantageous if the phase noise of a VCO used in a radio receiver could be reduced during the critical signal receive times. For example, if the VCO phase noise could be improved over the time required to receive a transmitted packet, then the system bit-error rate (BER) could potentially be reduced.

The motivation for this research is to explore a technique that allows for a noise reduction in a circuit during times in which a temporary reduction in noise is desired. This reduction is accomplished by segmenting the circuit into several sub-circuits that are connected via switches and bias retention circuitry. A VCO was chosen to demonstrate the switched-bias and bias retention circuitry that was developed as it lent itself well to a comparison with and without the additional circuitry. That is, the phase noise could be measured and compared for the various states of the circuitry (i.e. for a VCO with no additional circuitry, with the switch in the on state and with the switch in the off state). While the reduction in noise is not continuous, this technique could be useful for systems that have defined periods during which a reduction in noise is advantageous. 


\subsection{Objective}

The objective of this research was to reduce the phase noise in a voltage controlled oscillator (VCO) by using a switched-bias circuit which consists of an NMOS switch, and a MIM (metal-insulator-metal) capacitor used for bias retention. The switched-bias circuitry was designed in the $0.18 \mu \mathrm{m}$ CMOS technology, with a $1.8 \mathrm{~V}$ supply.

The VCO has been designed in [8] by Daniel Olszewski, and was used in this research to provide a baseline against which the VCO containing the switched-bias method could be compared. The VCO was designed to oscillate at $1.4 \mathrm{GHz}$, with a tuning range of approximately $17 \%$.

\subsection{Thesis Organization}

The thesis is organized in six chapters presented as follows:

Chapter 2 provides a background for the research. In particular a view of the effect phase noise has on a system and a discussion on other methods that has been researched in the area of VCO noise reduction is presented. This discussion is followed by a look at the intrinsic and extrinsic noise sources found in CMOS circuits.

Chapter 3 explores the design of the switched-bias circuit, comprised of an NMOS switch and a MIM capacitor. The method used to integrate this technique into a VCO and the design issues that have been addressed are presented.

Chapter 4 presents a detailed look at the design and implementation of the circuit, including simulations and layout. This discussion also includes a look at the simulation challenges encountered during the design process and how these challenges were overcome to obtain the simulation results that are presented.

Chapter 5 shows the measurement results, as well as a discussion of the printed circuit board $(\mathrm{PCB})$ design and the test setup.

Chapter 6 concludes the thesis with a summary of the noise reduction technique and the simulation that were obtained from this research. Research contributions are highlighted and a proposal for future work in this research area is presented. 


\section{Chapter 2}

\section{Background Information}

\subsection{System Level Requirements for Phase Noise}

As stated in [9] the VCO is one of the key contributors to the overall phase noise performance in a communication system, therefore to allow the system to achieve its greatest potential it is important to reduce the VCO phase noise as much as possible. One such communication system that can be affected by phase noise is the global system for mobile communication (GSM).

As defined in [10] GSM is a globally accepted standard for digital cellular communication. It uses a narrowband time division multiple access (TDMA) system. A GSM network consists of three major systems: the switching system (SS), the base station system (BSS), and the operation and support system (OSS). GSM uses a full duplex communication in three frequency bands: $900 \mathrm{MHz}, 1800 \mathrm{MHz}$ and $1900 \mathrm{MHz}$, with a channel bandwidth of $200 \mathrm{kHz}$.

GSM was chosen as a system level example for this research due to its stringent phase noise requirements. From [11] the phase noise specification for a voltage controlled oscillator in GSM system, at a $600 \mathrm{kHz}$ offset, is determined to be $-121 \mathrm{dBc} / \mathrm{Hz}$. This value assumes a needed signal to noise ratio (SNR) after downconversion of $9 \mathrm{~dB}$, and states that the minimum GSM signal level to be detected is $-102 \mathrm{dBm}$.

The phase noise of several LC oscillators was reviewed, and the phase noise for each one is listed in Table 2.1. In addition, this table also compares the power and complexity of each design. Table 2.1 gives the reader a sense of the measured LC oscillator phase 
noise values reported in recent literature. The complexity is measured in terms of the number of transistors required, and is considered relative to the other designs presented. A downwards arrow indicates a simplified circuit, a side arrow indicates no additional circuitry, and an upward arrow indicates a VCO with additional circuitry. It can be seen that by using the typical LC oscillator design, the GSM specifications will not be automatically met. For example, the LC oscillators presented in $[12,13]$ presently could not be used for a GSM system as they are, but would require some some extra circuitry or techniques to lower the phase noise. The oscillator presented in [2] does meet the specifications, but does not leave any room for a margin of error. Therefore these designs could benefit from a method to reduce phase noise, allowing them to better meet the GSM system requirements.

Table 2.1: Comparison of LC oscillator phase noise performance

\begin{tabular}{|c|c|c|c|c|c|}
\hline Ref. & Technology & $\begin{array}{c}\text { Center } \\
\text { Frequency }\end{array}$ & $\begin{array}{c}\text { Phase Noise } \\
\text { at offset }\end{array}$ & $\begin{array}{c}\text { Power } \\
\text { Dissipation }\end{array}$ & Complexity \\
\hline$[2]$ & $\begin{array}{l}0.25 \mu \mathrm{m} \\
\mathrm{CMOS}\end{array}$ & $1.8 \mathrm{GHz}$ & $\begin{array}{c}-121 \mathrm{dBc} / \mathrm{Hz} \\
\text { at } 600 \mathrm{kHz}\end{array}$ & $6 \mathrm{~mW}$ & $\downarrow$ \\
\hline [14] & $\begin{array}{l}0.25 \mu \mathrm{m} \\
\text { CMOS }\end{array}$ & $1.6 \mathrm{GHz}$ & $\begin{array}{c}-98.5 \mathrm{dBc} / \mathrm{Hz} \\
\text { at } 400 \mathrm{kHz}\end{array}$ & $59 \mathrm{~mW}$ & $\uparrow$ \\
\hline$[12]$ & $\begin{array}{l}0.35 \mu \mathrm{m} \\
\mathrm{CMOS}\end{array}$ & $6.53 \mathrm{GHz}$ & $\begin{array}{c}-98.4 \mathrm{dBc} / \mathrm{Hz} \\
\text { at } 1 \mathrm{MHz}\end{array}$ & $18 \mathrm{~mW}$ & $\uparrow$ \\
\hline$[13]$ & $\begin{array}{l}0.35 \mu \mathrm{m} \\
\text { CMOS }\end{array}$ & $2.6 \mathrm{GHz}$ & $\begin{array}{c}-110 \mathrm{dBc} / \mathrm{Hz} \\
\text { at } 5 \mathrm{MHz}\end{array}$ & $26 \mathrm{~mW}$ & $\leftrightarrow$ \\
\hline$[11]$ & $\begin{array}{l}0.4 \mu \mathrm{m} \\
\mathrm{CMOS}\end{array}$ & $900 \mathrm{MHz}$ & $\begin{array}{c}-108 \mathrm{dBc} / \mathrm{Hz} \\
\text { at } 100 \mathrm{kHz}\end{array}$ & $9 \mathrm{~mW}$ & $\leftrightarrow$ \\
\hline [15] & $\begin{array}{c}0.5 \mu \mathrm{m} \text { CMOS } \\
\text { CMOS }\end{array}$ & $900 \mathrm{MHz}$ & $\begin{array}{c}-110 \mathrm{dBc} / \mathrm{Hz} \\
\text { at } 200 \mathrm{kHz}\end{array}$ & $6.6 \mathrm{~mW}$ & $\downarrow$ \\
\hline$[16]$ & $\begin{array}{l}0.6 \mu \mathrm{m} \\
\text { CMOS }\end{array}$ & $1.8 \mathrm{GHz}$ & $\begin{array}{c}-110 \mathrm{dBc} / \mathrm{Hz} \\
\text { at } 500 \mathrm{kHz}\end{array}$ & $\begin{array}{c}\text { Not } \\
\text { Reported }\end{array}$ & $\leftrightarrow$ \\
\hline
\end{tabular}

For the case of LC oscillators that already meet the GSM specifications, such as the one presented in [11], a further reduction in phase noise would also be beneficial. The reduction in VCO phase noise could be applied to help minimize power requirements in other areas of the system.

The relationship between bit error rate (BER) and phase noise was examined in [17], 


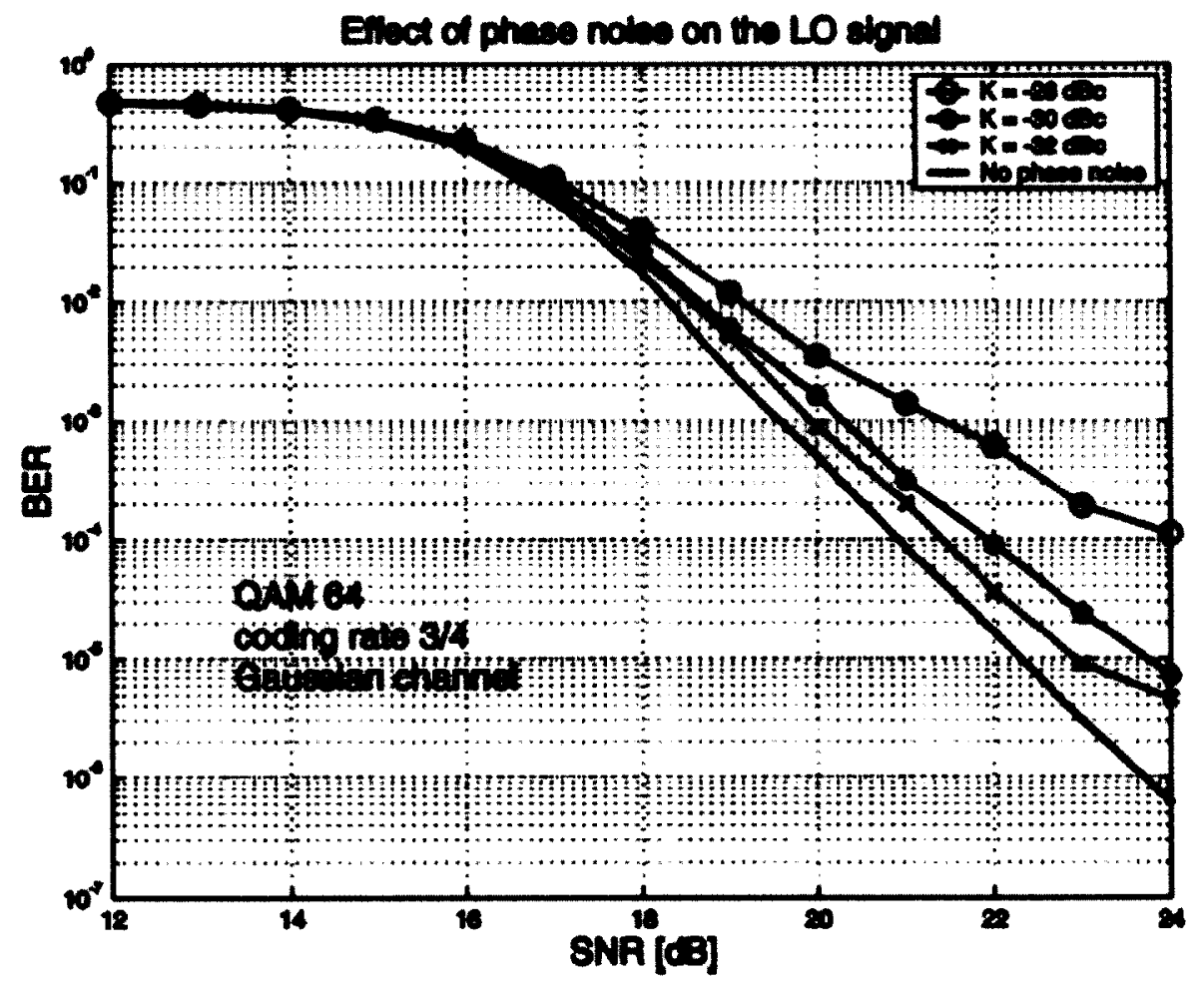

Figure 2.1: Relationship between BER and phase noise as shown in [17], with $B=10 \mathrm{kHz}$. This figure is reproduced with permission from the IEEE and Come et al., "Impact of front-end non-idealities on bit error rate performance of WLAN-OFDM transceivers", IEEE Radio and Wireless Conference 2000; (C) [2000] IEEE. 
and this relationship is plotted in Figure 2.1. In this research, the phase noise power density function (PDF) of a frequency synthesizer (FS) signal was approximated by a Lorenzian function with uniform phase distribution at a frequency, $\mathrm{f}$ [17]. The relationship defined by [17] is given by equation,

$$
L(f)^{2}=\frac{1}{\pi} \cdot \frac{K \cdot B}{f^{2}+B^{2}}+L_{0}
$$

where $\mathrm{f}$ is the frequency, $\mathrm{K}$ is the total integrated phase noise, $\mathrm{B}$ is the $-3 \mathrm{~dB}$ bandwidth, and $\mathrm{L}_{0}$ is the noise floor, which was set to $35 \mathrm{~dB}$ above the thermal noise. Figure 2.1 shows that as the phase noise, $\mathrm{K}$, increases, the BER also increases, illustrating the link between these two performance measurements.

The next section deals with other techniques that have been published to reduce phase noise in VCOs to provide the reader with some insight into other methods being researched in this area.

\subsection{Techniques Used to Reduce Phase Noise in VCOs}

\subsubsection{Elimination of the Bias Circuit in a VCO}

An interesting technique that has been reported to reduce phase noise involves removing the bias circuitry completely from the VCO circuit. This technique is discussed in [18]. A comparison was made between a LC-tuned voltage controlled oscillator (VCO) which contained a biasing network formed using a current mirror to produce the tail current, and a VCO which removed the tail current generator and placed the bias point to ground. The two circuits, as given in [18], are shown in Figure 2.2 and Figure 2.3.

As discussed in [18], by removing the bias network, it was expected that the VCO should show a lower phase noise, as the $1 / \mathrm{f}^{3}$ noise of the oscillator without the current generator can only originate from the flicker noise of the MOS transistor switches. It is explained in [18] that "the switches operate in triode region for large portions of the oscillation period and therefore exhibit lower current flicker noise than the tail transistor 


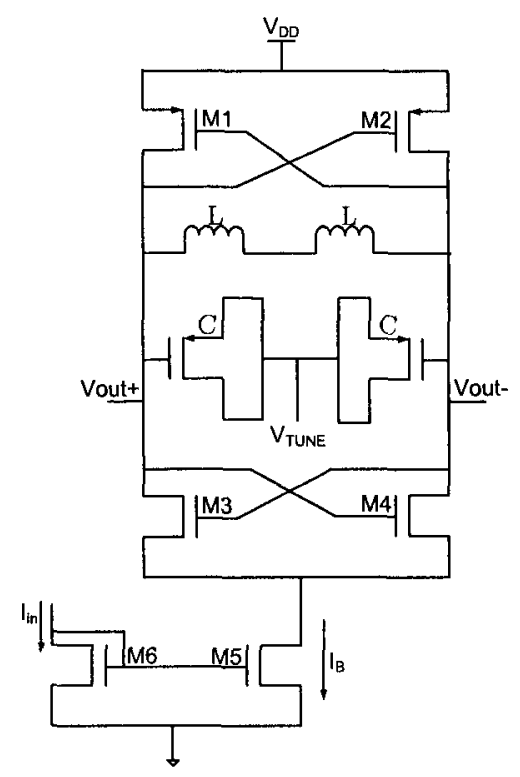

Figure 2.2: Schematic of the VCO containing a bias network to generate a tail current, as depicted in [18].

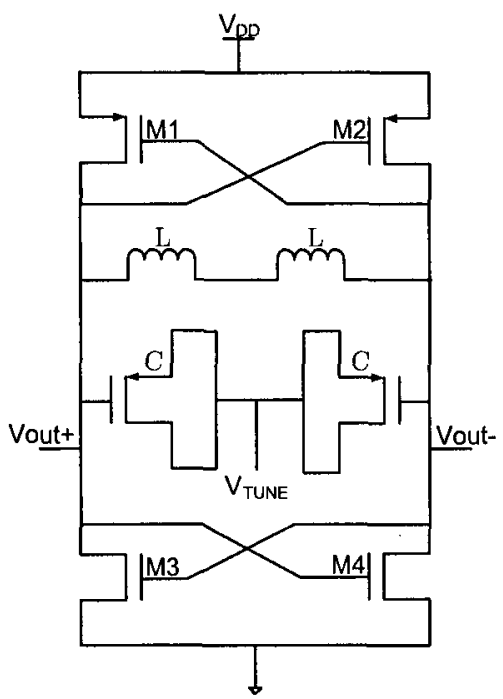

Figure 2.3: Schematic of a VCO without a bias network, as shown in [18]. 
that continuously operates in saturation." In addition, switched MOS transistors are known to have lower flicker noise than transistors biased in the stationary condition, which has been shown in [19].

It is discussed in [18] that the flicker noise of the switches was modelled by a voltage generator in series with the gate of each device. It is stated in [18] that this noise is in principle sampled by an impulse train at $2 \omega_{0}$, at the zero-crossing instants. As a result, the noise component at $\omega_{m}$ produces drain current tones at $\left(2 n \omega_{0} \pm \omega_{m}\right)$ for $n=\ldots,-$ $1,0,+1, \ldots$. It is stated in [18] that "the slow component of this current at $\omega_{m}$ modulates the bias voltage of the varactors. The oscillation amplitude is not affected because it is limited by the power supply." As a result, only the common-mode modulation to frequency modulation (CMM-to-FM) conversion mechanism of noise can take place using this topology. It was shown in [18] that the omission of the bias current generator from the oscillator resulted in a reported close-in phase noise improvement of $10 \mathrm{~dB}$ when compared to the same oscillator which contained the bias current generator.

A disadvantage encountered with this technique discussed by [18] is that the removal of the current source results in a higher sensitivity of the frequency with regards to the voltage supply (which is sometimes referred to as frequency pushing). In [18] it is suggested that this effect can be reduced through the use of a supply voltage regulator. The VCO without the bias generator required a higher power consumption in comparison to the oscillator with a bias generator $(2.9 \mathrm{~mA}$ compared to $2 \mathrm{~mA})$, however this balanced with the reduction in phase noise resulted in an improved typical figure of merit of the oscillators, which was defined in [18] as the product of the phase noise times power consumption (using $10 \mathrm{kHz}$ and $3 \mathrm{MHz}$ offsets).

This technique shows that the bias network does have an impact on the phase noise of a $\mathrm{VCO}$, as will be further shown within the switched-bias technique discussed in the next chapter of this thesis. 


\subsubsection{Filtering Noise}

Filtering of noise as described in [7] has been shown to reduce phase noise in an LC oscillator. In this technique a top-biased VCO is presented with a noise filter which is made from a capacitor and inductor, as shown in Figure 2.4. In [7] it was determined that for the current source that sets the bias level of the VCO only, the thermal noise in the current-source transistor around the second harmonic of the oscillation frequency causes phase noise. In addition it was observed that a high impedance at the tail is only required at the second harmonic to stop the differential-pair FETs in triode from loading the resonator. To combat the phase noise, a noise filter consisting of a capacitor and an inductor was designed. In [7] it was determined that "placing a large capacitor in parallel with the current source resulted in a shorting of the noise frequency around $2 \omega_{0}$ to ground." This statement means that the noise frequencies around $2 \omega_{0}$ were able to be suppressed using the technique presented in [7]. To achieve a higher impedance, an inductor was inserted between the current source and the tail. In [7] the inductance value was chosen to be in parallel with the capacitance at the common source of the differential pair, and so that it would resonate at $2 \omega_{0}$.

To further improve the design, a top-biased differential LC oscillator was designed in [7]. In this configuration, the current source is connected from the positive supply to the center tap of the differential inductor. This leads to a circuit that is more immune to substrate noise because the current source is placed in an n-well rather than in the substrate. As well, it is stated by [7] that the top-bias oscillator upconverts less flicker noise into phase noise.

The result from the fabricated chip with the filtering, as shown in Figure 2.5, indicated that there was an 8-dB improvement over the oscillator that did not contain the filter method. The measured phase noise $3 \mathrm{MHz}$ away from the $1.2 \mathrm{GHz}$ oscillation frequency was $-152 \mathrm{dBc} / \mathrm{Hz}[7]$.

The capacitor size required for this technique was a $40-\mathrm{pF}$ MIM capacitor. This is four times larger than the $10 \mathrm{pF}$ MIM capacitor used within the switched-bias technique discussed in the next chapter of this thesis. The switched-bias technique does incorporate 


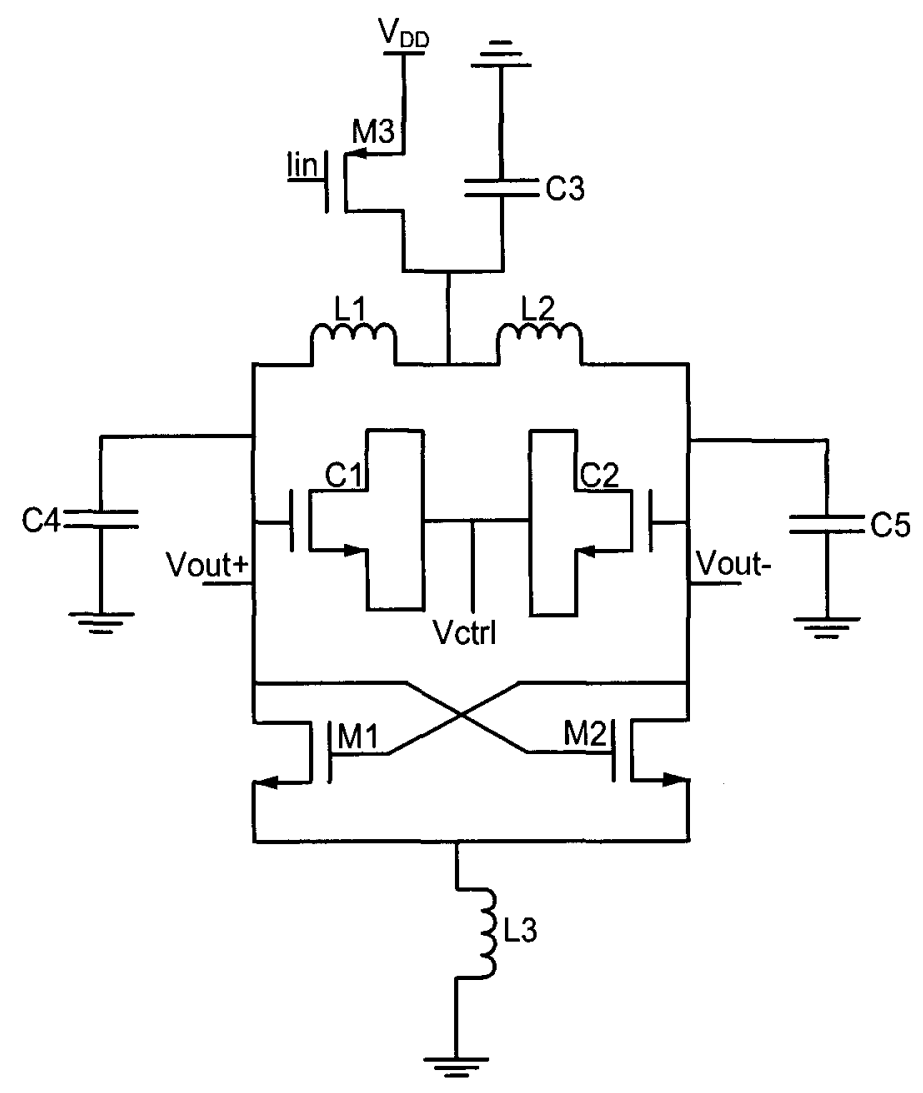

Figure 2.4: Top-bias VCO noise filtering technique introduced in [7]. 


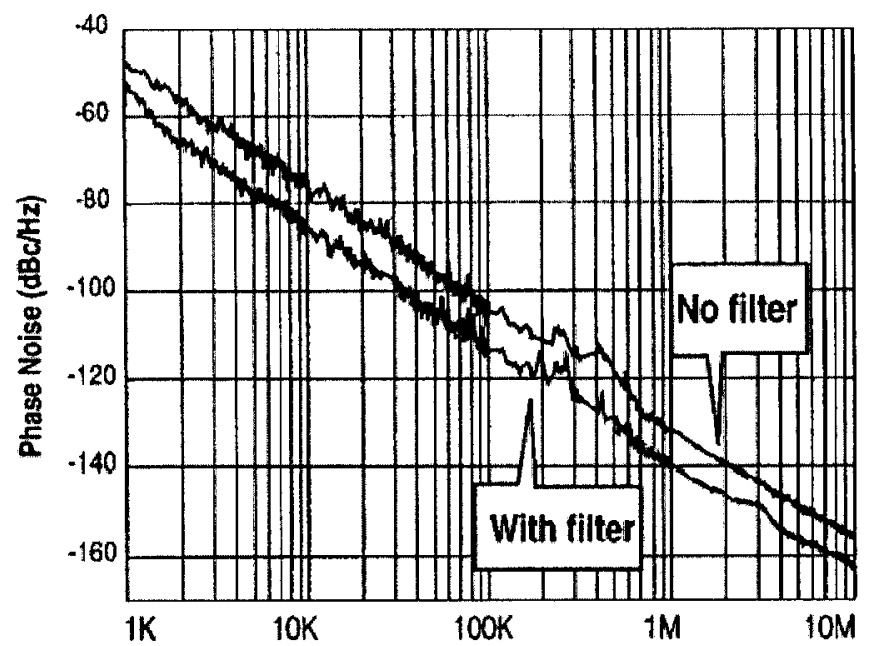

Figure 2.5: Measurement results comparing the top-bias VCO both with and without the noise filtering technique as shown in [7]. This figure is reproduced with permission from the IEEE and Hegazi et al., "A filtering technique to lower LC oscillator phase noise", IEEE Journal of Solid-State Circuits; (C) [2001] IEEE.

the idea of using a capacitive filter to reduce the phase noise in terms of the bias retention element which is in the form of the $10 \mathrm{pF}$ capacitor and helps in part to filter some of the noise from the bias network.

\subsubsection{Using Topology to reduce noise}

Focusing on the topology of the oscillator is another technique that has been used in the past to reduce phase noise. For example, LC oscillators have been shown to have lower phase noise than ring oscillators, as was discussed in [20]. The LC oscillator topology has also been further explored to try to further reduce phase noise as can be seen in [20-23]. The LC topologies have varied from a cascade coupling topology forming a quadrature VCO that can overcome IQ mismatch, as shown in [21] to a cross-coupled LC oscillator that uses on-chip spiral inductors and junction varactors in the resonance LC-tank, as presented in [20]. A summary of the phase noise results for several low-phase noise LC VCO topologies are presented in Table 2.2. Each topology will be discussed in more detail within this section. 
Table 2.2: Comparison of LC oscillator topologies used to reduce phase noise (PN). In terms of the change in phase noise, a down arrow indicates a decrease in phase noise was reported.

\begin{tabular}{|c|c|c|c|c|c|}
\hline $\begin{array}{l}\text { Topology } \\
\text { Description }\end{array}$ & Ref. & Technology & $\begin{array}{l}\text { Center } \\
\text { Freq. }\end{array}$ & $\begin{array}{c}\text { PN } \\
\text { at offset }\end{array}$ & $\begin{array}{c}\text { PN } \\
\text { Change }\end{array}$ \\
\hline $\begin{array}{l}\text { Cross-coupled LC VCO } \\
\text { using on-chip spiral } \\
\text { inductors and junction } \\
\text { varactors in the } \\
\text { resonance LC-tank }\end{array}$ & [20] & $\begin{array}{l}0.18 \mu \mathrm{m} \\
\text { CMOS }\end{array}$ & $2.4 \mathrm{GHz}$ & $\begin{array}{c}-100.2 \mathrm{dBc} / \mathrm{Hz} \\
\text { at } 100 \mathrm{kHz} \\
-131.4 \mathrm{dBc} / \mathrm{Hz} \\
\text { at } 3 \mathrm{MHz}\end{array}$ & $\downarrow$ \\
\hline $\begin{array}{l}\text { Cascode coupling; } \\
\text { new quadrature VCO }\end{array}$ & [21] & $\begin{array}{l}0.18 \mu \mathrm{m} \\
\text { CMOS }\end{array}$ & $6 \mathrm{GHz}$ & $\begin{array}{c}-115 \mathrm{dBc} / \mathrm{Hz} \\
\text { at } 1 \mathrm{MHz}\end{array}$ & $\begin{array}{c}\text { Not } \\
\text { Reported }\end{array}$ \\
\hline $\begin{array}{l}\text { LC VCO; uses series } \\
\text { combination of a } \\
\text { bondwire inductor and } \\
\text { a spiral inductor. } \\
\text { Tuning element is a } \\
\text { MOS varactor instead } \\
\text { of a p-n junction }\end{array}$ & [22] & $\begin{array}{l}0.35 \mu \mathrm{m} \\
\mathrm{CMOS}\end{array}$ & $1.3 \mathrm{GHz}$ & $\begin{array}{l}-119 \mathrm{dBc} / \mathrm{Hz} \\
\text { at } 600 \mathrm{kHz}\end{array}$ & $\begin{array}{c}\text { Not } \\
\text { Reported }\end{array}$ \\
\hline $\begin{array}{l}\text { LC VCO with negative } \\
\text { resistance formed } \\
\text { using a double-cross } \\
\text { connection with an } \\
\text { NMOS and PMOS } \\
\text { differential pair }\end{array}$ & [23] & $\begin{array}{l}0.18 \mu \mathrm{m} \\
\text { CMOS }\end{array}$ & $5.8 \mathrm{GHz}$ & $\begin{array}{c}-115 \mathrm{dBc} / \mathrm{Hz} \\
\text { at } 1 \mathrm{MHz}\end{array}$ & $\downarrow$ \\
\hline
\end{tabular}


In [20] a completely integrated $2.4 \mathrm{GHz}$ low-power low-phase noise VCO was implemented as shown in Figure 2.6. This implementation is a cross-coupled LC VCO using on-chip spiral inductors and junction varactors in the resonant LC-tank. The VCO-core draws $1.5 \mathrm{~mA}$ of current from a $1.2 \mathrm{~V}$ supply. The attractiveness of this method is shown by the results presented in [20] which demonstrate an excellent phase noise performance over recently reported results [24-27] while still achieving a lower power dissipation through a wide range of offset frequencies. The disadvantage of this topology is that it requires two spiral inductors which must be optimized, and require a large amount of area relative to the rest of the circuit.

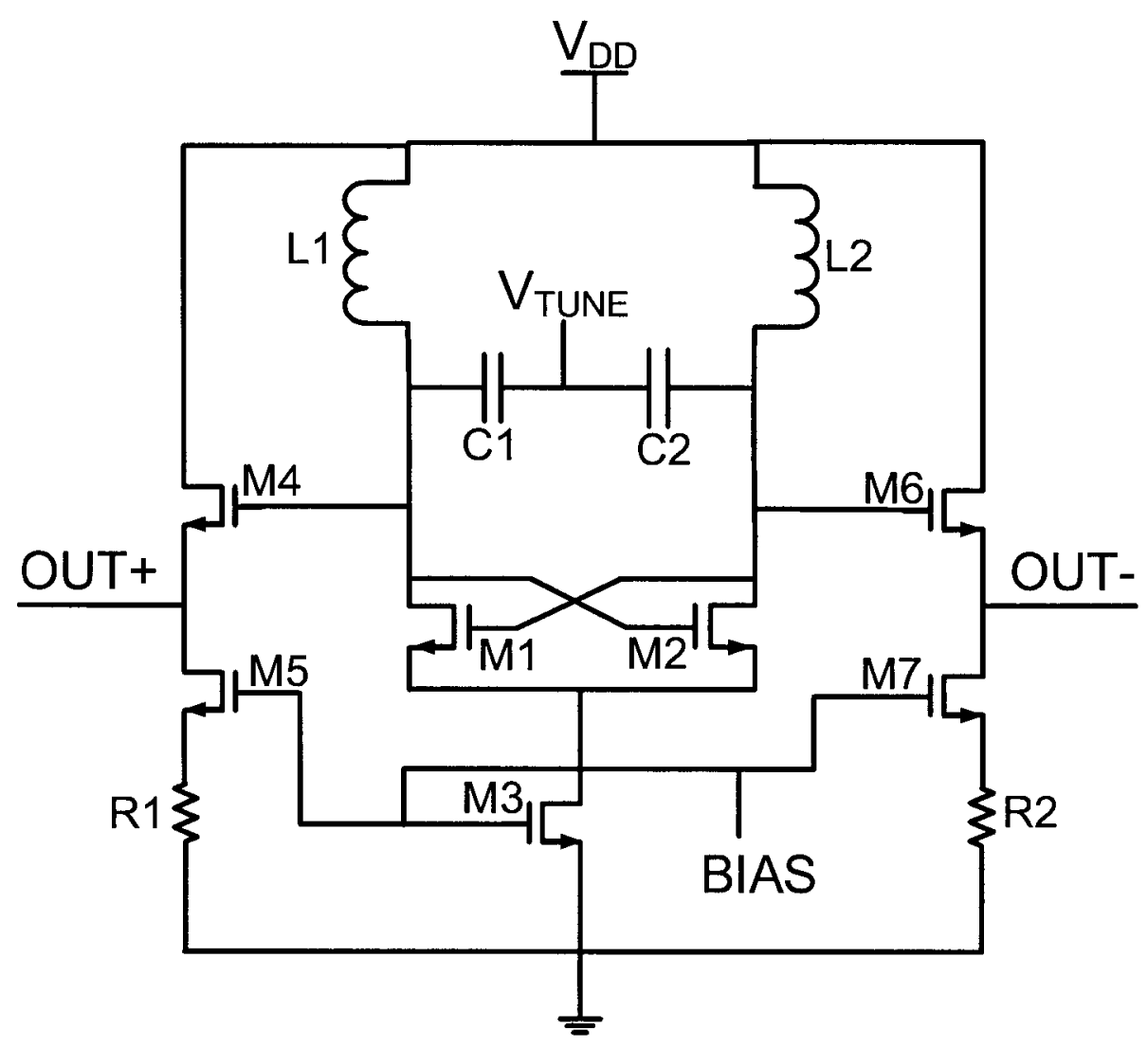

Figure 2.6: Low-power, low-phase noise VCO as presented in [20].

In [21] low-power and low-phase noise were achieved using a cascode coupling topology for a quadrature VCO. The total power consumption for the complete circuit (including the two VCO-cores and two VCO-buffers) was $18 \mathrm{~mW}$. This design achieves maximum 
signal swing by eliminating the current source, which also helps reduce phase noise. However, the disadvantage in omitting the current source is the increased sensitivity to the power supply noise, which was addressed by using a cascode coupling configuration. The main disadvantage of this technique is its level of complexity, as it requires implementing two VCO-core and buffer circuits (see Figure 2.7) instead of a single circuit. Not only does the second VCO increase the complexity of the design but it will also increase the area required to implement this design.

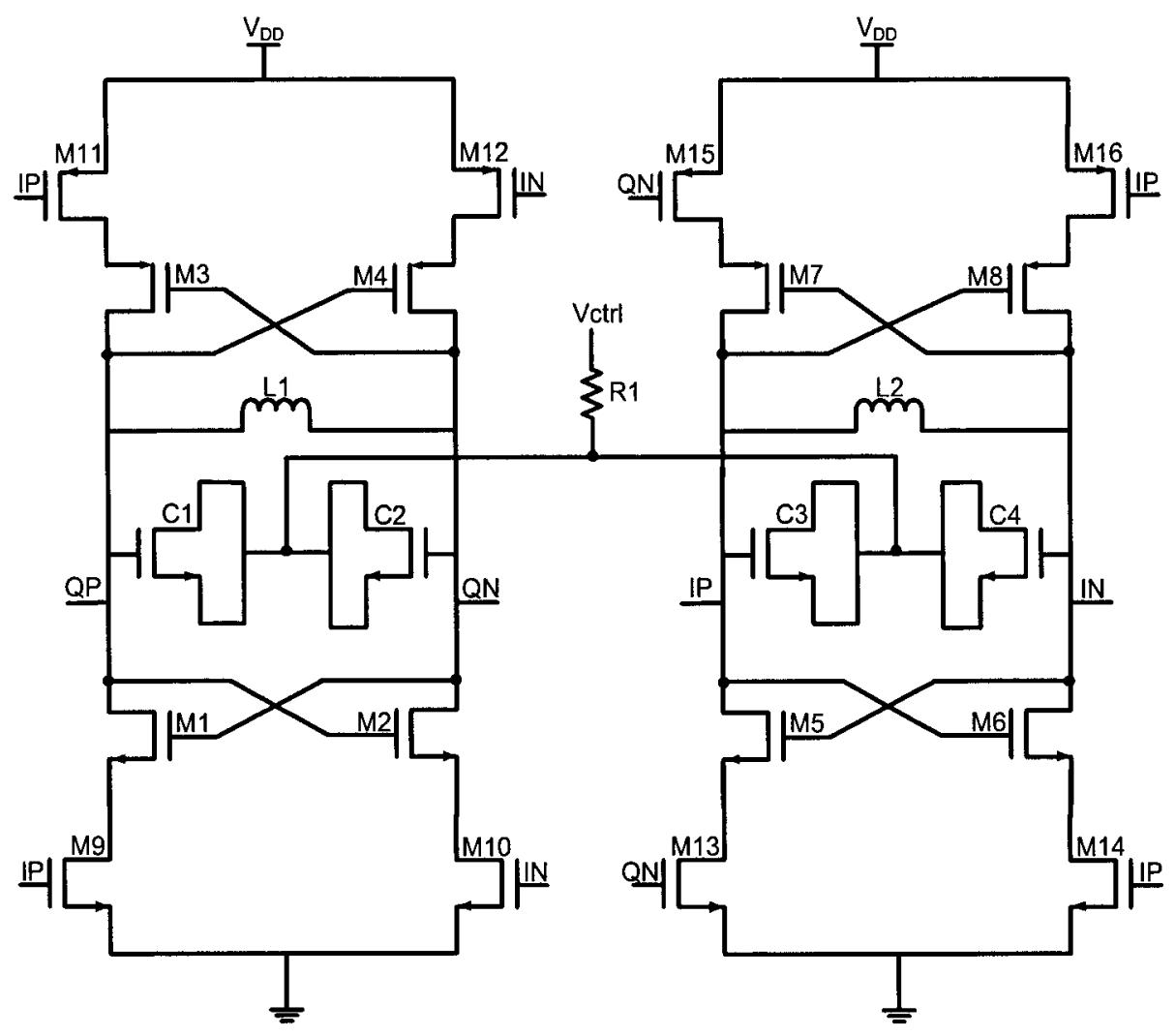

Figure 2.7: Quadrature VCO schematic proposed in [21].

An LC VCO which uses a series combination of a bondwire inductor and a spiral inductor is presented in [22], and shown in Figure 2.8. In this design the tuning element is a MOS varactor instead of a p-n junction. The advantage stated by [22] of this design is that the use of the MOS varactor allows for a larger tuning range for a given applied voltage, when compared to the use of a p-n junction for the tuning element. In addition, 
the quality factor of the composite inductor used is shown to be higher than that of a spiral inductor. Lastly, the research in [22] has shown that the C-V characteristics of the MOS varactor results in a smaller variation of the VCO gain for a given tuning range, as the MOS varactor is intrinsically more linear than a $\mathrm{p}-\mathbf{n}$ varactor. The disadvantage of the design is that if only bondwire inductors are used, the phase noise will be heavily dependent on the quality factor of the varactor. As the MOS varactors allow a high tuning range within a small voltage range, the result is that the VCO gain can become excessively high, especially for high oscillation frequencies. This can be a problem, as discussed in [22], because of the high sensitivity to the varactor control voltage that results, and leads to the result that any noise coupled to the varactor control electrode is translated into a large phase noise at the oscillator output. Extra circuitry in the form of a fixed value capacitor would be required to try and rectify this problem.

The last research work that will be considered in this section is the work discussed in [23]. An LC VCO with negative resistance formed using a double-cross connection with an NMOS and PMOS differential pair was presented in [23] and is shown in Figure 2.9. The total power consumption for this design is $6.23 \mathrm{~mW}$. This design achieves a superior phase noise in comparison to previous works, while still having a very low power consumption. A drawback for this configuration is the large parasitic capacitance of the PMOS which results in a decrease in the tuning range. In addition, the use of multiple inductors will greatly increase the chip area required to implement the design on chip.

In addition there has been work done at a system level to reduce noise. In [28] a method using correlation and a precise phase stabilization technique is discussed, while [29] focuses on the idea of VCO realignment to reduce phase noise.

While these methods do result in a reduction in phase noise they have a narrow focus in that only a particular topology is considered. The research presented in this thesis investigates a general technique to reduce phase noise in VCOs. The details of this research will be discussed in the next chapter. 


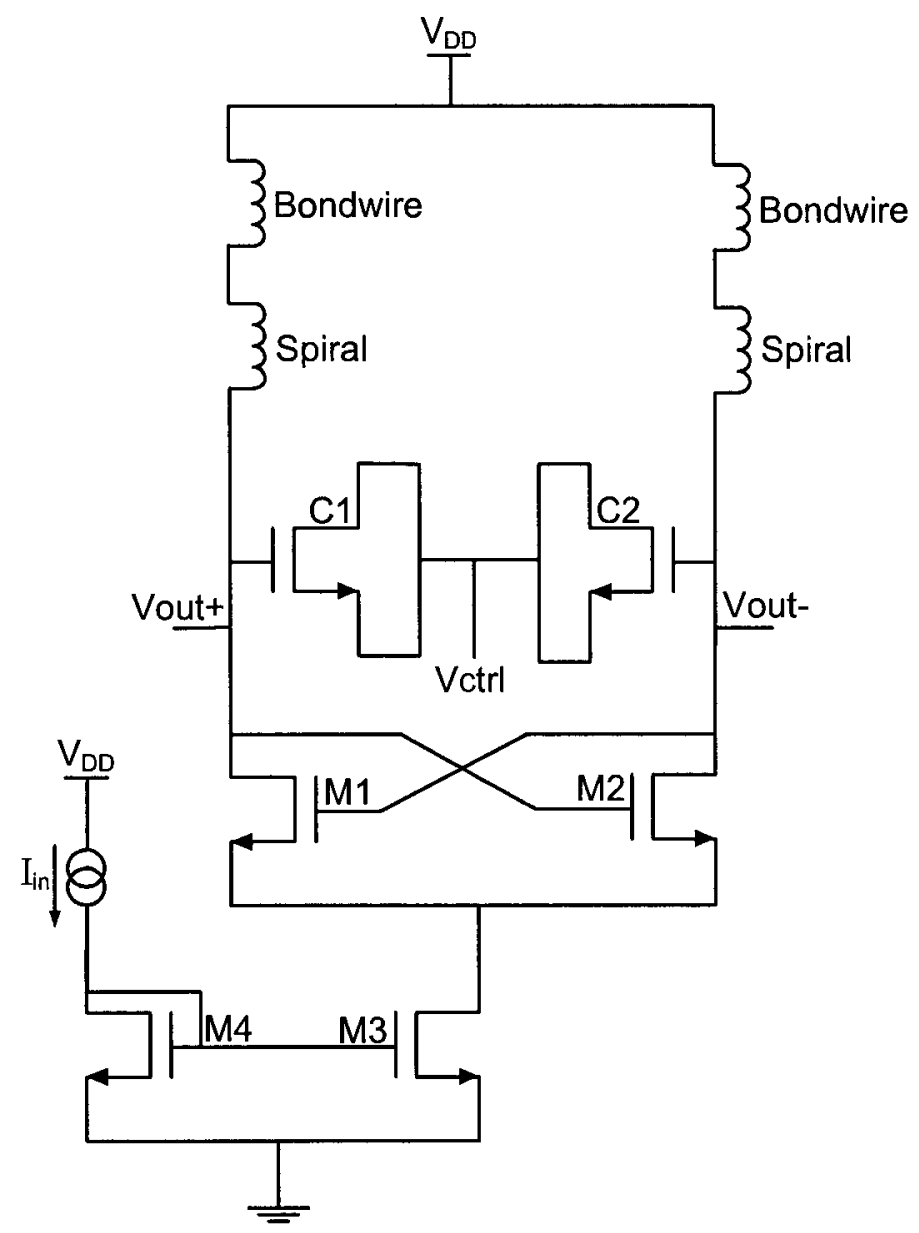

Figure 2.8: LC CMOS VCO given in [22]. The inductor is realized as the series combination of spiral and bondwire inductors. 


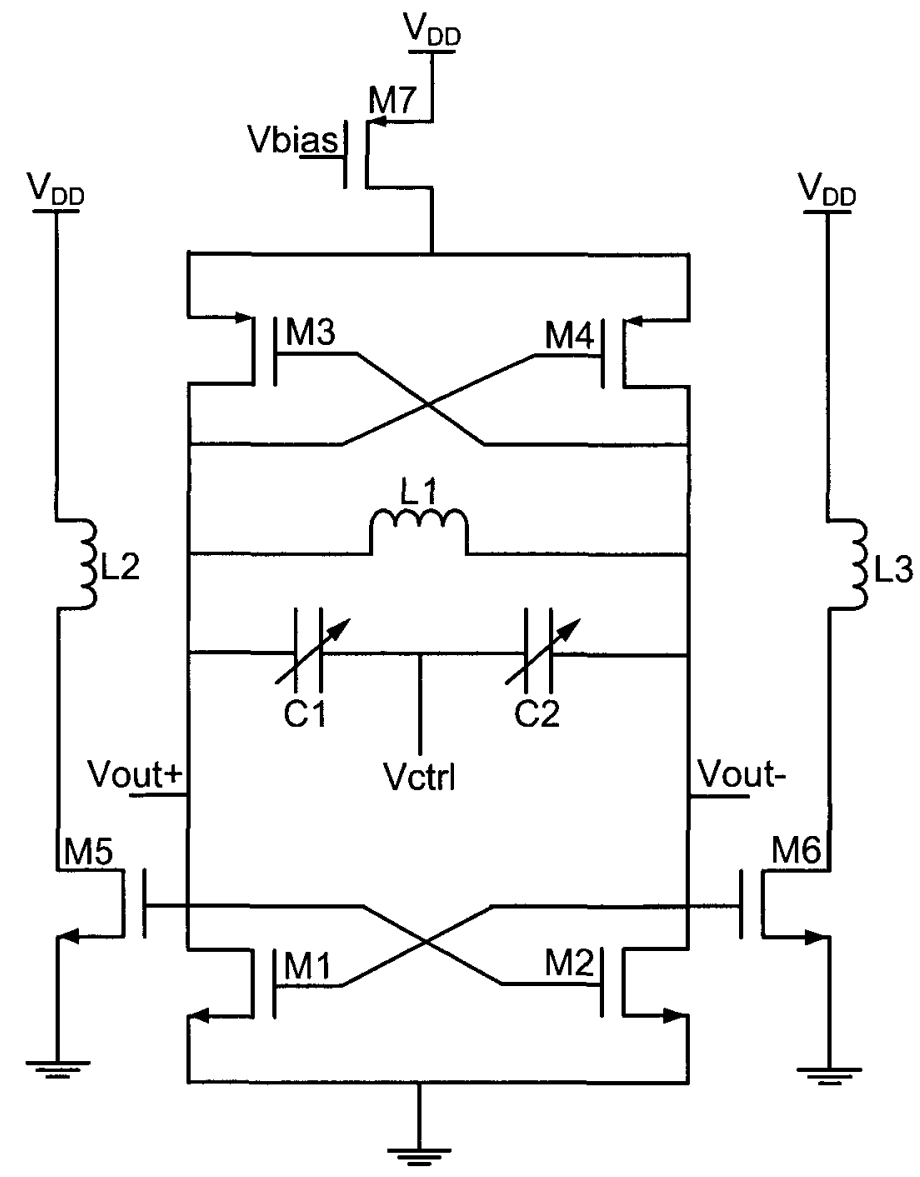

Figure 2.9: Schematic of the LC VCO with negative resistance as shown in [23]. 


\subsection{CMOS Intrinsic Noise Sources}

Intrinsic noise is noise that arises within a circuit. Complementary Metal Oxide Semiconductor (CMOS) circuits have several intrinsic noise sources that must be considered when designing a CMOS circuit. Two of the main noise sources are thermal noise and flicker noise, which will be further elaborated on in this section. The main model used to model thermal and flicker noise in a transistor is shown in Figure 2.10.

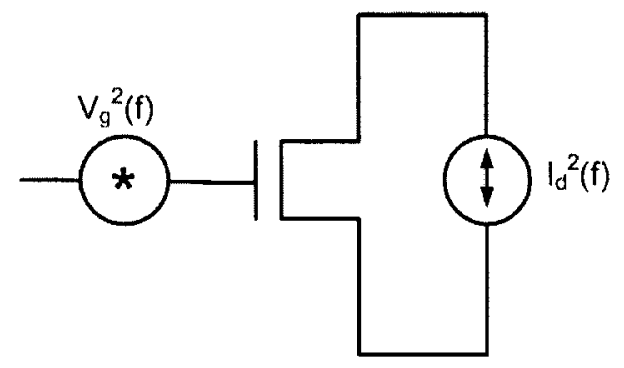

Figure 2.10: Intrinsic noise sources for a MOSFET in the saturation region of operation.

\subsubsection{Thermal Noise}

As stated in [30] the thermal noise in the channel of a MOSFET in saturation is given by equation,

$$
I_{n t g}^{2}=4 k T\left(\frac{2 g_{m}}{3}\right)
$$

where $\mathrm{k}$ is Boltzmann's constant, $\mathrm{T}$ is the temperature in Kelvin and $\mathrm{g}_{m}$ is the smallsignal transconductance at the bias point.

However, as the derivation of thermal noise for a transistor depends on its mode of operation, if a transistor is in the triode region the thermal noise is given by equation, .

$$
I_{n t g}^{2}=\frac{4 k T}{r_{d s}}
$$

where $\mathrm{k}$ is Boltzmann's constant, $\mathrm{T}$ is the temperature in Kelvin, and $\mathrm{r}_{d s}$ is the channel resistance [31, pp.200-201]. 
The focus of this thesis is on reducing the close-in phase noise of a VCO through the integration of switched-bias circuit into the bias network of the VCO. As thermal noise dominates a larger offset frequencies (refer to Figure 2.13), it will not be considered further in this thesis.

\subsubsection{Flicker Noise}

The flicker noise (also known as $1 / \mathrm{f}$ noise) in MOSFETs can be determined by the relationship shown by equation

$$
V_{g}^{2}(f)=\frac{K}{W L C_{o x} f}
$$

where $\mathrm{K}$ is a constant which depends on device characteristics, $\mathrm{W}$ and $\mathrm{L}$ are the transistor's width and length, respectively, $\mathrm{C}_{o x}$ is the gate capacitance per unit area, and $\mathrm{f}$ is the frequency [31, pp.200]. This equation for flicker noise uses the model of a voltage source in series with the MOSFET gate, as was shown in Figure 2.10 and defined in $[31$, pp.200].

As can be seen from (2.3.3), the flicker noise is inversely proportional to the transistor gate area, $\mathrm{W} \times \mathrm{L}$; therefore larger devices generally have less flicker noise. As discussed in [31, pp.200] flicker noise is important in MOSFET circuits because it generally dominates at low frequencies. The relationship between phase noise and frequency is shown in Figure 2.13 .

\subsection{CMOS Extrinsic Noise Sources}

Many present day chips contain both analog and digital circuitry. This can lead to extrinsic noise problems, which, as discussed in [32, pp.28-29], can be defined as the disturbances which are caused by the natural operation of neighbouring blocks in the circuit that appear in other parts of the circuitry. It is usually the switching of digital circuitry that interferes with the more sensitive analog circuits. The two main ways presented in [32, pp.29] that switching noise is coupled to other circuitry is through the 
power supply and substrate channels. In addition, the bias network can be considered external from the main circuit, and it also couples in noise. The bias is an extrinsic noise source that will be the focus of this thesis.

\subsubsection{Power Supply Noise}

As mentioned in [32, pp.29-30] the connections required to join an integrated circuit to an off-chip power and reference node introduce a finite impedance between the circuitry and the on-board supply's power (VDD) and ground (GND) terminals, as can be seen in Figure 2.11. As stated in $[32$, pp.29] when the digital circuit sources/sinks current from/to the rails a transient is induced on $\mathrm{VDD}_{\text {on-chip }}$ and $\mathrm{GND}_{\text {on-chip }}$ due to the resistive (IR) and inductive (di/dt disturbances) voltage drops. Any signal or noise on the supply lines will couple into the active circuitry through stray capacitances and the gain of the bias network and will be amplified by the active circuitry. These unwanted signals are noise and degrade the circuit's performance [33].

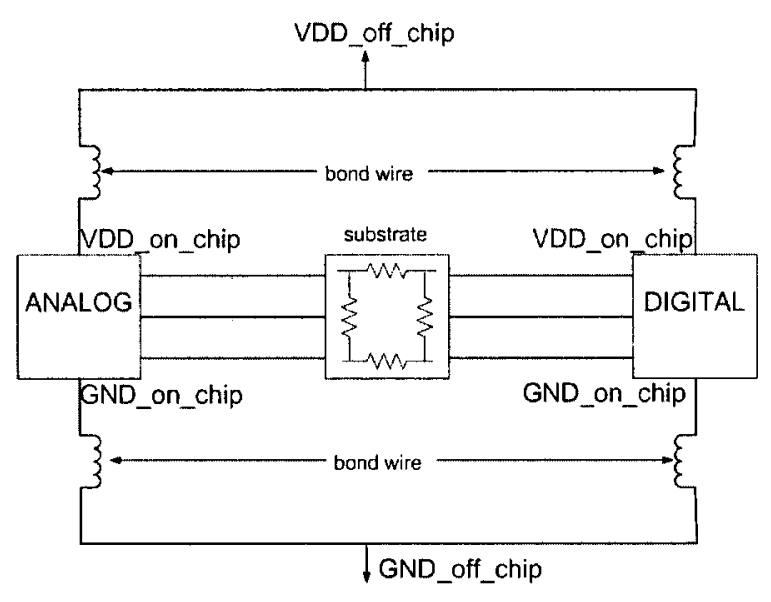

Figure 2.11: Diagram showing a mixed-signal chip which indicates the on chip and off chip supply voltages, which are connected by bondwires, as presented in [32].

It is desirable to try and minimize the noise that is coupled into the circuitry. By design, in digital logic circuits are largely immune to the variations they introduce on the supplies [32, pp.31]. However, this is generally not the case with standard analog circuits. There are several methods that are used to help reduce the amount of supply 
noise seen by the analog circuitry. The main techniques include isolating the analog and digital blocks from each other, reducing the switching noise in the circuit, and designing noise resistant circuits.

One approach to isolate the analog and digital circuitry is to have separate power pins for each block. However, there is still capacitive coupling between the on-chip power grids and inductive coupling between the off-chip bondwires. An alternative method to improve isolation is to increase the number of power and ground pins. This lowers the effective inductance of the off-chip VDD and GND, but does increase the complexity of the packaging. In addition, coupling capacitors should be placed between the on-chip VDD and GND pins. This helps reduce the amount of noise introduced on the lines. As discussed in [32, pp.32] when a noise current passes through one bondwire some of it will be routed by the capacitor into the other pin, thus splitting the effect of the noise.

Another common approach to reducing the impact of noise in an integrated circuit is to use a differential configuration. Within a limited region of operation this configuration allows fluctuations on the rails to excite the circuit in a common-mode fashion only. This property degrades as the input swings on the gates increase (resulting in an unbalanced circuit) or due to mismatch between the devices. Also, this approach is generally less useful at high frequencies due to the effect of parasitics.

\subsubsection{Substrate Noise}

The noise that results from the switching of the transistors is also injected into the substrate. The substrate noise can influence the noise in the bias circuit, and it can be coupled into the bias lines. Methods, such as those suggested for the power supply noise, can be used to help reduce the effect of the substrate noise when designing analog and digital circuits.

The effect of substrate noise on VCOs was studied in detail in [34]. The study presented in [34] showed that "the phase noise of a VCO is adversely affected by substrate noise. In the extreme, the VCO can lock to the substrate noise." That is, if the noise is large enough close to the VCO's tank resonant frequency, then there is the potential the VCO 
will lock on the substrate noise, which is offset from the VCO's required center frequency. It has been shown in [34] that guard rings can be used to "effectively attenuate substrate noise at lower frequencies" but that "their effectiveness degrades at higher frequencies due to circuit parasitics." In [34] it was shown that, at $900 \mathrm{MHz}, 25 \mathrm{~dB}$ of isolation could be obtained, while the isolation was reduced to $10 \mathrm{~dB}$ at $5.2 \mathrm{GHz}$. Therefore substrate noise continues to be a problem for mixed-signal design. Though there was no digital circuitry, besides the switch in the switched-bias circuit, included on the chip with the VCO, the design practices mentioned in subsection 2.4.1, such as using multiple ground and power supply pads, was still followed to help reduce the amount of substrate noise appearing in the VCO.

\subsubsection{Bias Noise}

A source of noise in analog circuits comes from the active and resistive components contained in the bias generation circuitry. As mentioned in [18] even low frequency noise (for example flicker noise) produced in the bias circuitry can have an adverse effect on the operation of high frequency circuits, such as VCOs. When the bias reference circuit is located far from the biased circuit, noise can be coupled into the bias supply line and consequently into the biased circuit. If the bias generation circuitry provides a reference current that is scaled upwards through current mirroring, the contributed noise is also scaled upwards.

While differential structures can reduce the effect of bias circuit noise, circuits undergoing large signal operation, such as VCO circuits, are still adversely affected.

\section{$2.5 \mathrm{kT} / \mathrm{C}$ Noise}

As defined in $[35, \mathrm{pp} .723-724] \mathrm{kT} / \mathrm{C}$ noise is the noise associated with the gate capacitor of a MOSFET. In [35, pp.723-724] it is stated that the maximum RMS output noise that can be generated from a simple RC circuit is given by equation,

$$
\sqrt{k T / C}
$$


where $\mathrm{k}$ is Boltzmann's constant, $\mathrm{T}$ is the temperature in Kelvin, and $\mathrm{C}$ is the capacitor value. This type of noise is often discussed with regards to Sample-and-Hold circuitry, which has a similar setup to the circuitry used for the switched-bias method discussed in this thesis. Figure 2.12 shows how $\mathrm{kT} / \mathrm{C}$ noise can occur in a circuit.

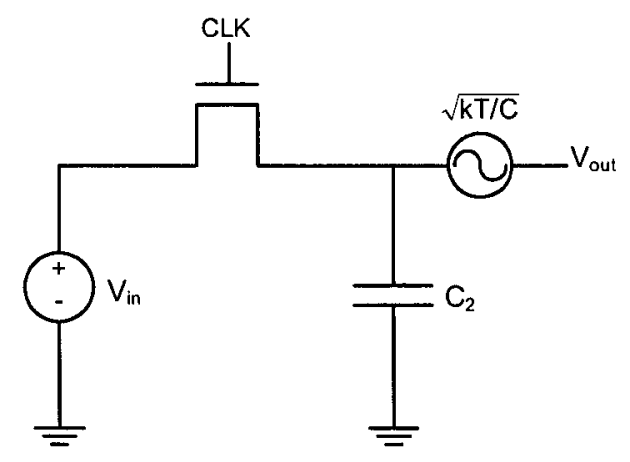

Figure 2.12: Model of $\mathrm{kT} / \mathrm{C}$ noise in a circuit.

The MOSFET shown in Figure 2.12 can be thought of as a resistor (when the MOSFET is turned on), which allows for the addition of the RMS noise source in series with the output of the capacitor. The noise can be considered to be a sampled voltage onto the capacitor each time the switch is turned on [35, pp.723-724]. From [35, pp.723] it is seen that the RMS noise generated when using a $1 \mathrm{pF}$ capacitor is $64 \mu \mathrm{V}$, while a $100 \mathrm{fF}$ capacitor results in a noise voltage of $200 \mu \mathrm{V}$. This indicates that the larger the size of the capacitor, the smaller the noise voltage sampled on the storage capacitor. For this particular thesis, a $10 \mathrm{pF}$ capacitor was used, which, assuming a temperature of 300 degrees Kelvin, and using the equation above, the $\mathrm{kT} / \mathrm{C}$ noise is found to be $20.3 \mu \mathrm{V}$. As stated in [30], $\mathrm{kT} / \mathrm{C}$ is related to thermal noise as is really a "repackaging of thermal noise." As was previously mentioned in the thermal noise section (section 2.3.1) the focus of this thesis is on close-in phase noise, and as a result the $\mathrm{kT} / \mathrm{C}$ noise will not be further considered as it relates to the thermal noise and the thermal noise floor, which dominate at larger frequencies.

However, there is a trade-off between speed and noise when it comes to high-speed systems, as smaller capacitors require less time to charge. Therefore, as mentioned in [35, pp.723], this tradeoff between high-speed/low-noise must be made when selecting the capacitor size. 


\subsection{Phase Noise}

For VCOs one of the main performance measurements is phase noise. Phase noise (PN) is defined in [36, pp.246] as "any noise that changes the frequency or phase of an oscillator waveform." Phase noise is given in $[36, p p .246]$ by "PN $=\mathrm{P}_{0} / \mathrm{N}_{0}$, where $\mathrm{P}_{0}$ is the power in the tone at the frequency of oscillation and $\mathrm{N}_{0}$ is the noise power spectral density at some specific offset from the carrier. Phase noise is generally measured in $\mathrm{dBc} / \mathrm{Hz}$ " [36, pp.246]. It is also stated in [36, pp.246] that "is desirable to reduce phase noise because phase noise results in power in the 'skirts' that form around a desired frequency. The result is that these 'skirts' can result in a reduction in the accuracy of measurements that depend on a frequency reference".

Phase noise is important in a VCO, for example in a radio receiver as mentioned in [37, pp.271], because the phase noise in the local oscillator will limit the immunity the receiver has against nearby interference signals. The phase noise in an oscillator may overwhelm nearby weak channels. As discussed in [37, pp.272] this is due to the fact that the "phase noise spectral density grows directly with the transmitted signal power, and at a given point in space, the noise sidebands of a strong transmitter may be greater than another weaker signal occupying the same frequency."

\subsubsection{Leeson's Equation}

Leeson's equation has historically been a generally accepted method for determining phase noise. The noise prediction using Leeson's model is based on the time-invariant properties of the oscillator, such as the resonator $\mathrm{Q}$, the feedback gain, the output power, and the noise figure.

Leeson's phase-noise equation, as defined in [38, pp.18-19], is given by equation,

$$
L(\Delta \omega)=10 \log \left[\left(\frac{2 F k T}{P_{\text {sig }}}\right)\left\{\left(\frac{\omega_{0}}{2 Q \Delta \omega}\right)^{2}+1\right\} \cdot\left(\frac{\Delta \omega_{1 / f^{3}}}{|\Delta \omega|}+1\right)\right]
$$

where $\mathrm{L}\{\Delta \omega\}$ is the single sideband (SSB) noise spectral density in units of $\mathrm{dBc} / \mathrm{Hz}$, $\mathrm{k}$ is Boltzman's constant, $\mathrm{T}$ is temperature in Kelvin, $\mathrm{P}_{\text {sig }}$ is the oscillator signal power, 
$\omega_{0}$ is the oscillation frequency, and $\Delta \omega$ is the offset from $\omega_{0}$. The quality factor $Q$ is the loaded $Q$ of the oscillator resonator, $F$ is the noise figure of the oscillator, and $\Delta \omega_{1 / f^{3}}$ is the corner frequency between the $1 / \mathrm{f}^{3}$ and $1 / \mathrm{f}^{2}$ regions.

In Leeson's model $\Delta \omega_{1 / f}$ is equal to the $1 / f$ noise corner frequency of the device. However, in practice, as stated in $[38, \mathrm{pp} .20], \Delta \omega_{1 / f^{3}}$ is rarely equal to the $1 / \mathrm{f}$ noise corner of the device. In addition, the noise figure of an oscillator is extremely difficult to predict, therefore the factor $\mathrm{F}$ is in reality a correction factor that can only be determined by the measurement of the phase noise spectrum of the oscillator. Since $\mathrm{F}$ and $\Delta \omega_{1} / f^{3}$ must generally be measured from the oscillator spectrum, it makes it difficult to use (2.6.1) to predict methods to reduce phase noise. However, this method is can be used as a starting point to predict phase noise.

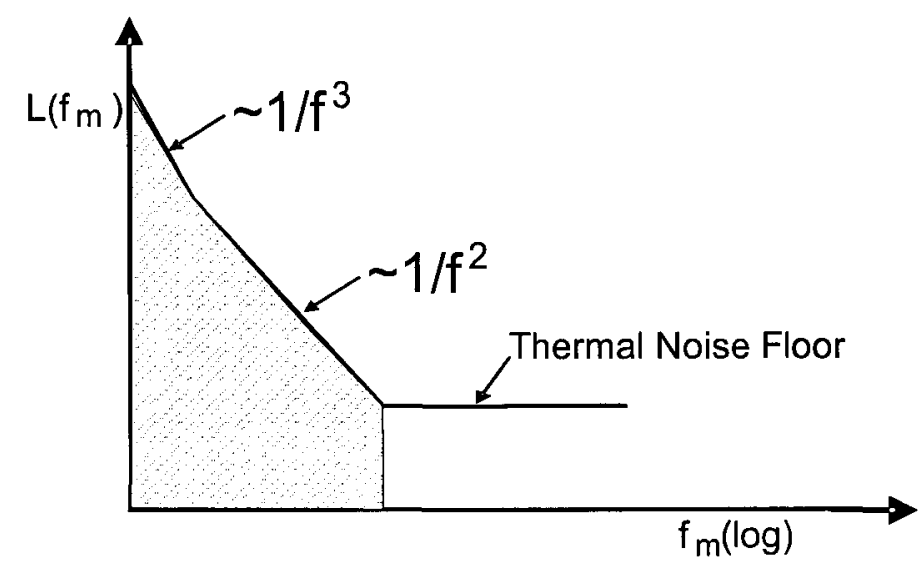

Figure 2.13: Illustration of the phase noise versus frequency relationship. The flicker noise is shown by the $1 / \mathrm{f}^{3}$ portion of the plot.

The shaded area shown in Figure 2.13 shows the range of offset frequencies that were concentrated on during this research. The switched-bias technique is directed towards reducing the amount of flicker noise within phase noise by disconnecting a portion of the bias network. The bias network is made up of MOSFETs which contribute flicker noise to the overall phase noise, therefore by removing MOSFETs from the circuit, the overall phase noise should decrease. 


\subsection{Chapter Summary}

- This chapter described the role of phase noise within a system, and how it relates to bit-error rate (BER).

- Next, the focus shifted to a discussion on various techniques that have been proposed in literature to reduce phase noise in VCOs.

- A brief overview of intrinsic and extrinsic noise sources that affect MOSFET circuits was presented.

- Lastly, a definition of phase noise and Leeson's equation were discussed. 


\section{Chapter 3}

\section{Switched-Bias Method and Implementation}

\subsection{Switched-Bias Method}

\subsubsection{Overview of the Switched-Bias Method}

As discussed in [2] a VCO can be constructed using a differential pair and an LC network. Biasing circuitry is used to ensure that the transistors remain in the correct mode of operation. However, the biasing circuitry also generates noise which is coupled into the main circuit, reducing the VCO's overall performance. For applications where the output can be sampled, it may be beneficial to disconnect the biasing network around the sampling time, resulting in lower effective noise value. Temporarily removing the biasing circuitry should not negatively impact the operation of the circuit.

The idea of segmenting circuits into bias control, bias retention, and processing elements is illustrated in Figure 3.1. In Figure 3.1 the elements are connected with idealized switches, enabling the elements to be selectively disengaged from the overall circuit. In this particular illustration, a bias retention element is directly connected to each processing element. Alternatively, the bias retention elements could be switched from both sides and completely disengaged from the processing circuits. 


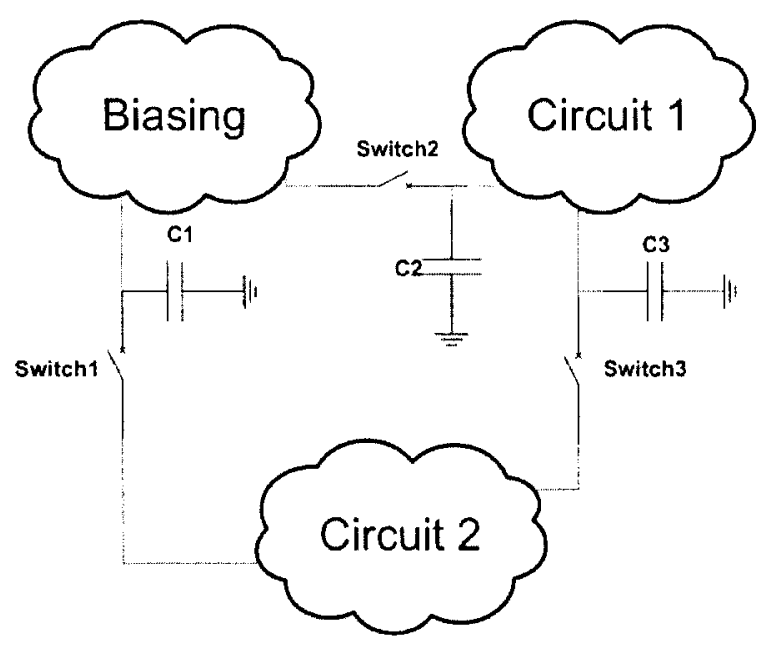

Figure 3.1: Diagram illustrating the circuit segmenting method. The elements are connected with idealized switches and the capacitors implement the bias retention elements.

\subsection{Implementation}

\subsubsection{Switched-bias circuit}

The switched-bias circuit consists of an NMOS switch, and a MIM (metal-insulator-metal) capacitor used as the bias retention element. The switching and bias retention elements are shown in Figure 3.2. The capacitor, C2, holds the charge when the switch is opened. Due to charge leakage at the bias retention node, the bias voltage will tend to change with time. The size of the storage capacitor and the frequency at which the bias voltage is reset to a level within operational specifications influences the variation of the bias voltage. The basic relationship relating current, capacitance and voltage is used to determine the size of the capacitor required as the bias retention element. The derivation is given by equations (3.2.1) to (3.2.5), as shown below:

$$
\begin{aligned}
& V(t)=V_{0}+\frac{1}{C} \int_{0}^{t} i_{l e a k} d t \\
& V(t)=V_{0}+\frac{1}{C}\left(i_{\text {leak }} t\right)
\end{aligned}
$$




$$
\begin{aligned}
& |\Delta V|=\frac{i_{\text {leak }} t}{C} \\
& \Delta V=\frac{i_{\text {leak }}}{C \cdot f_{c l k}} \\
& C=\frac{i_{\text {leak }}}{\Delta V \cdot f_{c l k}}
\end{aligned}
$$

where $\mathrm{i}_{\text {leak }}$ is the leakage current, which is assumed to be constant, $\Delta V$ is the bias variation allowed at the bias retention node, $V_{0}$ is the initial voltage and is assumed to be zero, $\mathrm{C}$ is the capacitance, and $f_{c l k}$ is the frequency at which the switch is toggled. It should be observed that in reality the leakage current will not be constant, but this calculation was used to obtain a rough estimate of the capacitance value, $\mathrm{C} 2$, required in the circuit, and simulations were done to verify the value obtained from (3.2.5), as will be discussed in subsection 3.2.2. A $10 \mathrm{pF}$ capacitor was chosen for the switched-bias circuit implemented in the VCO. This value was calculated assuming a $1 \mathrm{MHz}$ switch clock frequency, a voltage variation of $3 \mathrm{mV}$, which was obtained from simulations, and a $30 \mathrm{nA}$ leakage current. The leakage current is much higher than what is expected for an analog circuit, which, from [39], is expected to be within the pA range. However it was desired to overestimate the leakage to ensure a sufficiently large capacitor was used to hold the bias voltage value at the required level.

The voltage variation at the charge retention node is a function of time. The voltage can be reset when it crosses a threshold level, or it can be periodically reset, in order to restrict the variation to an acceptable range. Periodically resetting the retention node voltage is similar to the techniques used in DRAM circuits to periodically refresh the data held on storage capacitors. The duty cycle used for the voltage reset phase does not have to be $50 \%$. The clock pulse duration needs to be long enough to allow the voltage reset to occur, but should not be so long in duration that the amount of noise coupled to the 


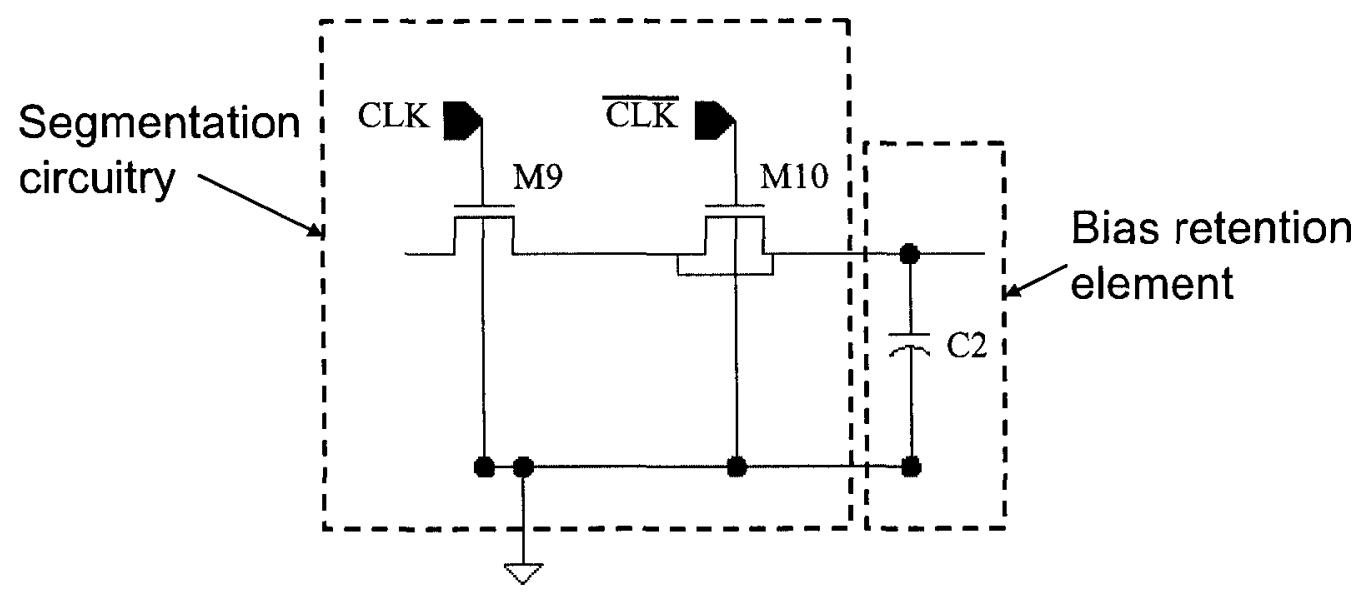

Figure 3.2: Switching and bias retention elements. M9 is an NMOS switch, M10 is a dummy switch used to reduce charge injection, and $\mathrm{C} 2$ is the bias retention element.

VCO is unnecessarily increased. An illustrative clock frequency of $1 \mathrm{MHz}$ was used for the VCO presented in this thesis to achieve the balance mentioned above.

As the switch element was implemented using an NMOS pass transistor, charge injection should be considered. To reduce this problem, a dummy switch, M10, was used in series with the main switch, M9, as shown in Figure 3.2. The dummy switch is controlled by an inverted version of the clock signal. As half of the channel charge is injected towards the dummy switch, M10, when M9 turns off, M10 is made half the size of M9. Although M10 is effectively shorted, a channel can still be induced by applying a voltage on the gate. As shown in [35, pp.722-723], this fact allows the charge injection of M9 to be essentially matched by the charge accepted by M10, cancelling the net charge injection to the susceptible signal processing element.

\subsubsection{Switch and Capacitor Sizing}

Three possibilities were considered for the switch used in the switched-bias circuit. The choices using typical MOSFETs consisted of an NMOS switch, a PMOS switch, or a transmission gate. The benefits of using a CMOS switch as described in [35, pp.719] include that fact that under DC conditions, the gate of the MOSFET does not draw current, therefore the gate control signal does not interfere with the information being 
passed through the switch. From these three options an NMOS switch was chosen as it required the minimum amount of area and complexity of the three switches. Simulation results presented later in this thesis indicate that the addition of the switch and bias retention circuitry had a low impact on the overall phase noise. However, if area was not a concern, than a PMOS switch would further reduce the noise associated with the switch. As stated in [31, pp.232] PMOS transistors have less 1/f noise than NMOS transistors, as the PMOS majority carriers (holes) have less potential to be trapped in surface states (which is one of theories as to the cause of flicker noise). A transmission gate would result in the lowest possible resistance of the three switches, as well as passing a logic high or a logic low value without a threshold drop [35, pp.719]. However, this type of switch is more complex than either a PMOS or an NMOS switch on its own as it requires both a clock and its complement, in addition to additional area to accommodate both the NMOS and PMOS transistors.

The size of the switch and the bias retention capacitor required for the design were determined through simulation. The length of the switch was kept constant at $0.38 \mu \mathrm{m}$. A length larger than the minimum length was used to help reduce potential variations due to fabrication and short channel effects. Design guidelines for the MOSFET length required to reduce the effects of channel length modulation are presented in [35, pp.228] and state that "the length of MOSFETs used in analog applications should be set to two to five times the minimum gate length." For the switch NMOS, the length was set to just over two times the minimum length to meet this guideline. In addition, MOSFETs with larger areas have been shown in [40] to have lower flicker noise, providing another reason to have a length larger than minimum size.

Using the testbench shown in Figure 3.3, the width of the switch, M9, as is denoted by $\mathrm{W}$ in the testbench, was swept between $1 \mu \mathrm{m}$ and $100 \mu \mathrm{m}$, in $2 \mu \mathrm{m}$ steps. The bias retention capacitor was held constant at $10 \mathrm{pF}$ and the phase noise of the $\mathrm{VCO}$ at a $100 \mathrm{kHz}$ offset was observed using PSS/Pnoise simulations (plots at offsets of $600 \mathrm{kHz}, 1 \mathrm{MHz}$ and showing a plot with the offset swept $100 \mathrm{kHz}$ to $1 \mathrm{MHz}$, were also generated but for simplicity have not been included as they show the same trend as was observed with an offset of $100 \mathrm{kHz}$ ). 

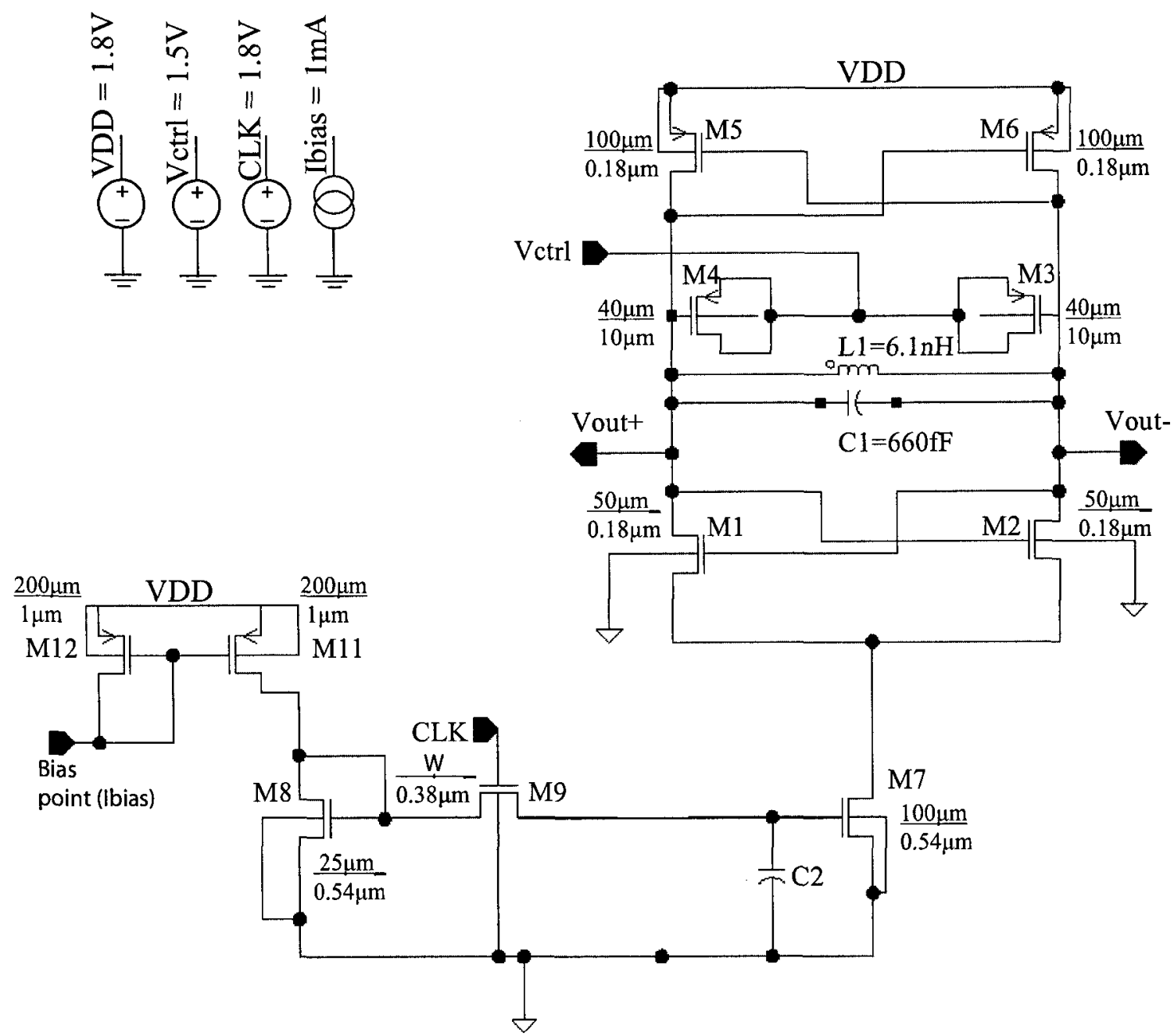

Figure 3.3: Test setup used to determine the switch, M9, width and the size of the capacitor, C2, required for the switched-bias circuit. 


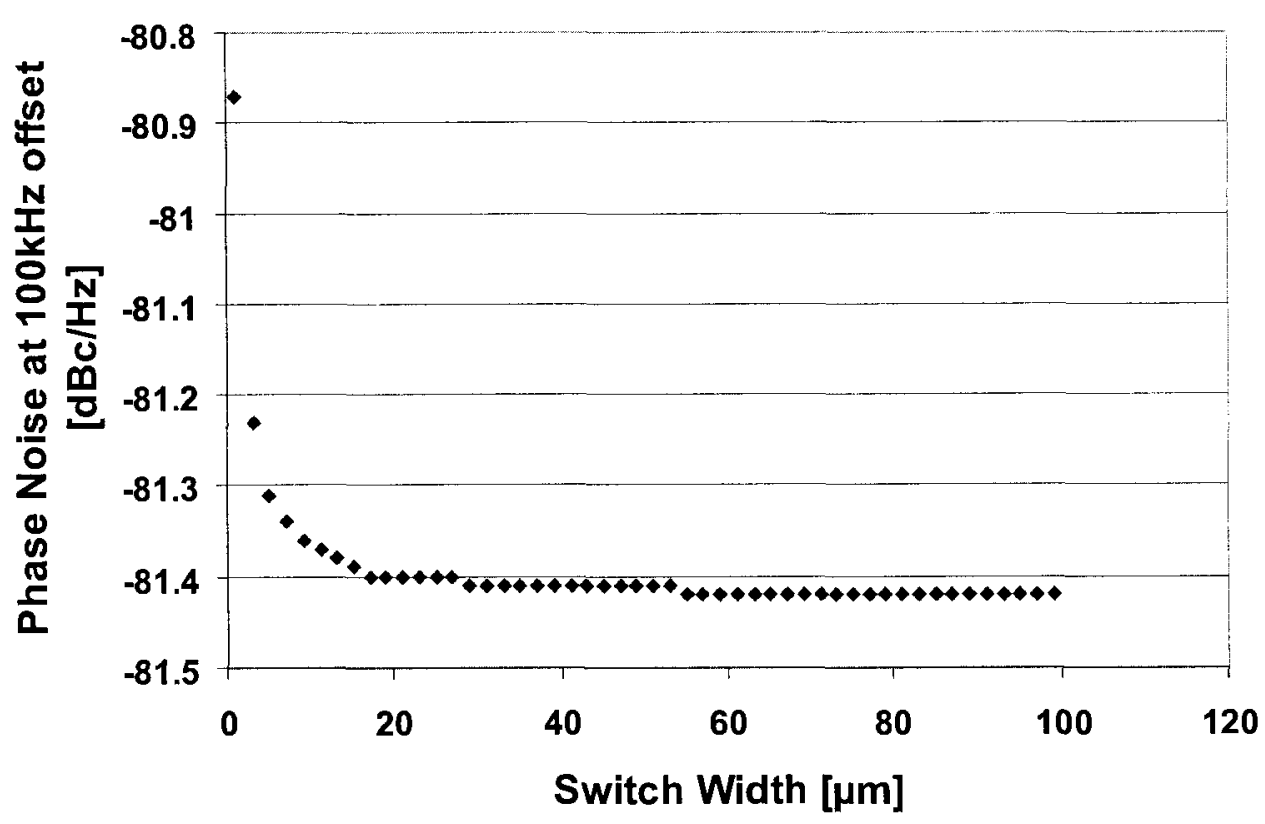

Figure 3.4: Plot showing the switch width being swept from $1 \mu \mathrm{m}$ and $100 \mu \mathrm{m}$, in $2 \mu \mathrm{m}$ steps, as the phase noise of the VCO at $100 \mathrm{kHz}$ offset is measured.

The results from the parametric sweep are shown in Figure 3.4, and it can be observed that after about $10 \mu \mathrm{m}$ the plot begins to level off, indicating that there is no additional benefit obtained by further increasing the width beyond this point.

Next the size of the bias retention capacitor was swept from $1 \mathrm{pF}$ to $100 \mathrm{pF}$ as the switch, M9, width and length were kept constant at $10 \mu \mathrm{m}$ and $0.38 \mu \mathrm{m}$, respectively, using the same test setup that was used to determine the switch width. Though a $100 \mathrm{pF}$ integrated capacitor would not be used on chip as it would require to much area and would not be practical, the simulation was swept to this value to see the impact capacitor size has on phase noise. It was desired to see if there would be a large improvement at much larger capacitor sizes. For the phase noise of the oscillator at the $100 \mathrm{kHz}$ offset, it was observed that while the phase noise does decrease as the capacitor size increases, the difference in phase noise between the $5 \mathrm{pF}$ and $100 \mathrm{pF}$ capacitor is only $0.18 \mathrm{~dB}$, as is shown in Figure 3.5 .

To ensure the trend of decreasing phase noise with increasing capacitor size, the phase 


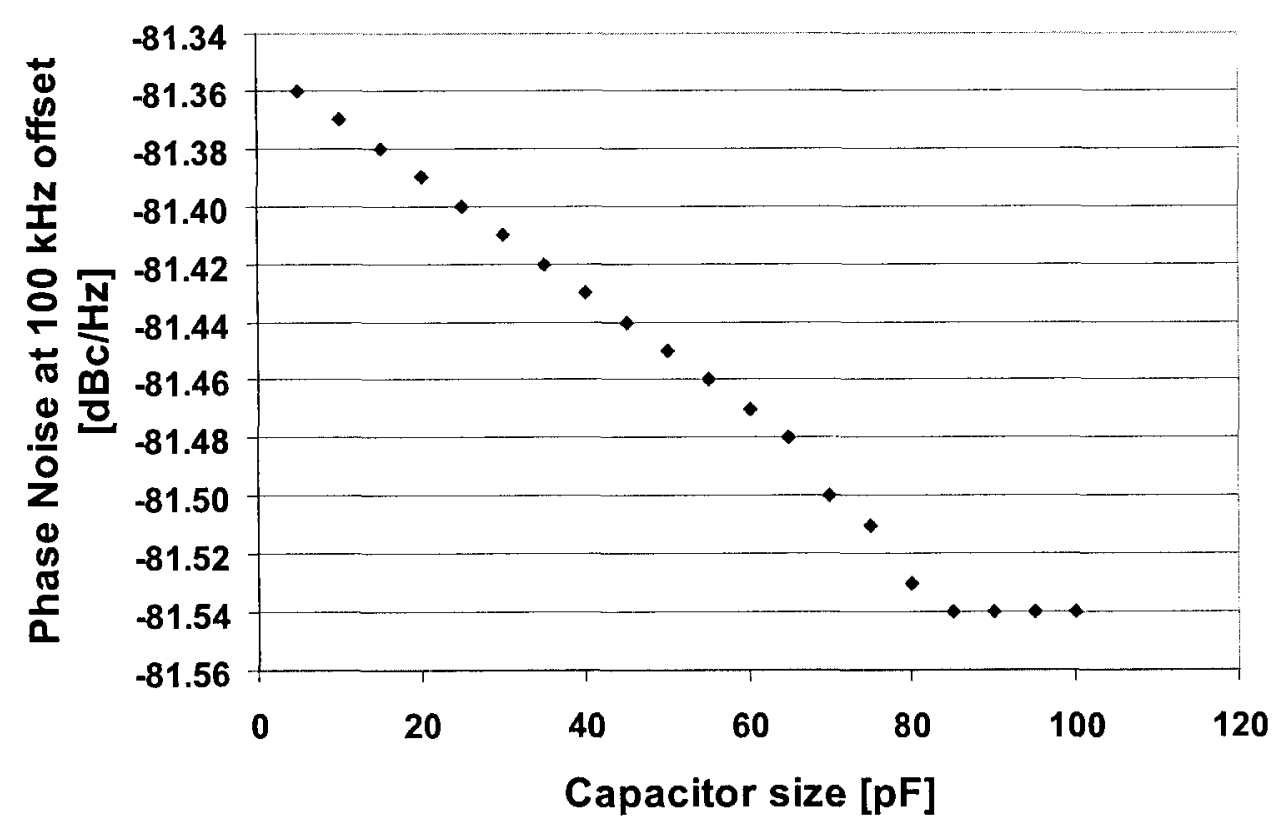

Figure 3.5: Plot showing the capacitor size being swept from $1 \mathrm{pF}$ and $100 \mathrm{pF}$, in $5 \mathrm{pF}$ steps, as the phase noise of the VCO at a $100 \mathrm{kHz}$ offset is measured.

noise of the oscillator at $600 \mathrm{kHz}$ and $1 \mathrm{MHz}$ offsets were also plotted, as can be seen in Figure 3.6 and Figure 3.7, respectively. It is observed in these plots that there is more of a step function, and there is a larger change in the phase noise between the case for the $5 \mathrm{pF}$ capacitor and the $100 \mathrm{pF}$ capacitor. For a frequency offset of $600 \mathrm{kHz}$ the decrease in phase noise is $0.3 \mathrm{~dB}$, while for a frequency offset of $1 \mathrm{MHz}$, the decrease in phase noise is $0.5 \mathrm{~dB}$. This indicates that at the higher frequencies the larger capacitor size does have more of an impact on filtering noise in the circuit. However, the decrease in phase noise in all three cases are still not large enough to justify the extra space required to use a larger capacitor in this particular circuit. As the chip area available was at a premium it was decided to try to keep the capacitor as small as possible, while still benefiting from a lower phase noise. It was determined that a $10 \mathrm{pF}$ capacitor would be the best size for this particular application, as it allowed the benefit of some filtering of the phase noise, while not taking up a large amount of area (the capacitor occupied approximately $11100 \mu \mathrm{m}^{2}$, or $1.1 \%$ of the total chip area), and still allow a big enough capacitor to hold the charge 


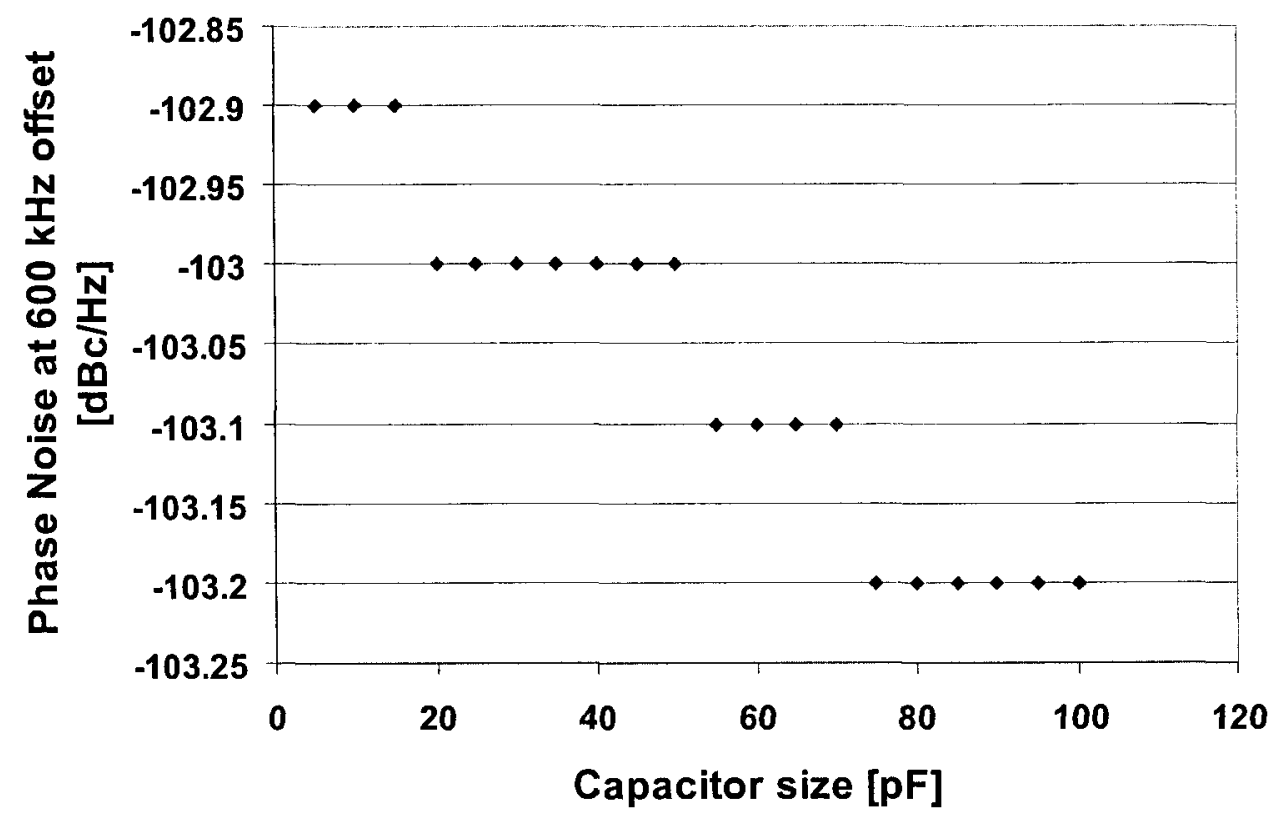

Figure 3.6: Plot showing the capacitor size being swept from $1 \mathrm{pF}$ and $100 \mathrm{pF}$, in $5 \mathrm{pF}$ steps, as the phase noise of the VCO at a $600 \mathrm{kHz}$ offset is measured.

at the gate of M7 in the bias network when the switch is turned off, as was discussed in subsection 3.2.1.

\subsection{Design Issues}

There are a number of design issues that must be taken into consideration when the switched-bias circuit is being implemented. These considerations include the bias voltage variation and the sizing of the current mirror.

\subsubsection{Voltage Variation at Bias Node}

Ideally a capacitor should hold charge for an infinite amount of time. In reality, a capacitor has a current leakage rate associated with it. In terms of the switched-bias circuitry, the capacitor forms the bias retention portion of the circuit. This element is vital to the circuitry as it maintains the correct operation of the VCO when the switch is opened. 


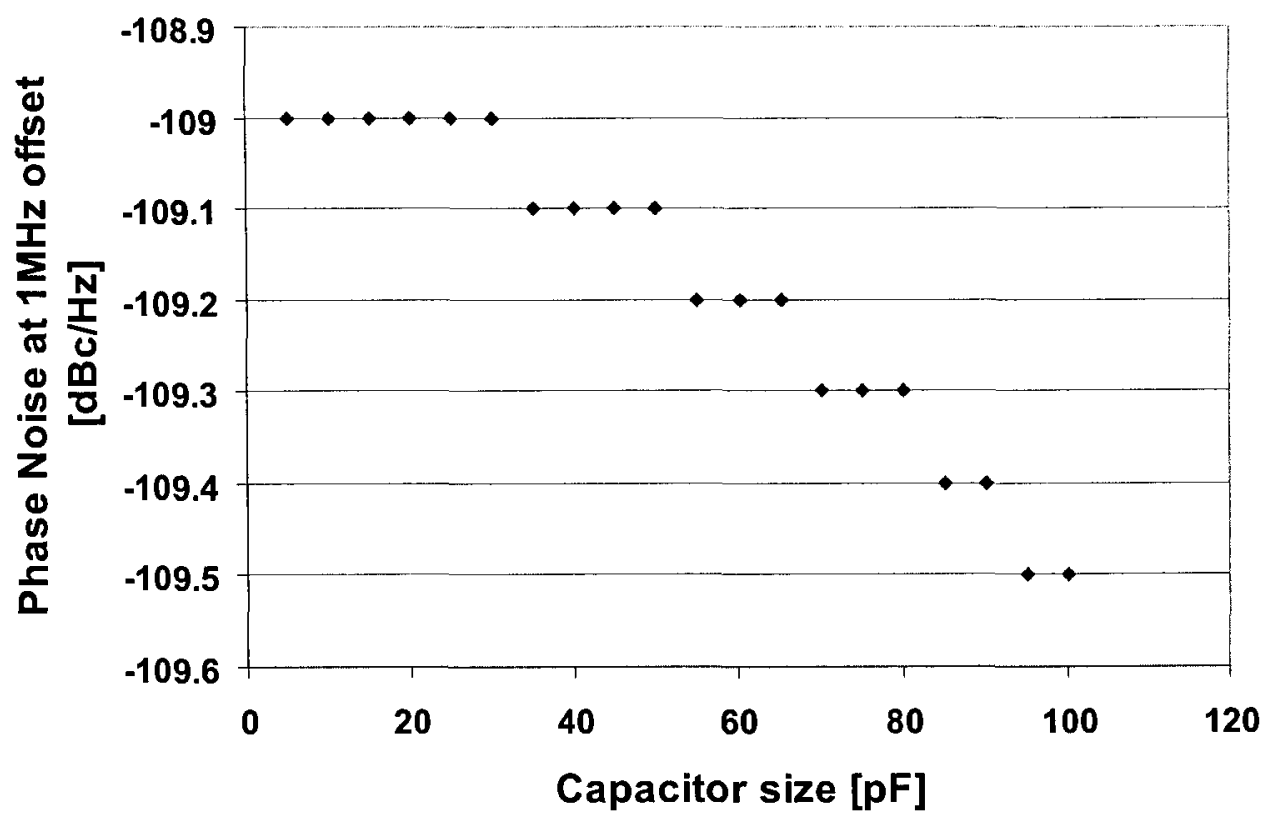

Figure 3.7: Plot showing the capacitor size being swept from $1 \mathrm{pF}$ and $100 \mathrm{pF}$, in $5 \mathrm{pF}$ steps, as the phase noise of the VCO at a $1 \mathrm{MHz}$ offset is measured.

The current leakage rate will impact the clock frequency required by the switch. The longer the capacitor can hold the voltage within the voltage range required to properly bias the circuit, the longer the switch can remain open, resulting in a longer interval in which the phase noise will be at a reduced level.

To evaluate the impact of the voltage variation, simulations were done to observe the effect at the bias node at the gate M7 (see Figure 3.8) and, as result, on the frequency at the output of the VCO. A transient parametric sweep simulation was run in Cadence using SpectreRF. The testbench for the simulation is shown Figure 3.8. The transient simulation was run for $1 \mu \mathrm{s}$ to ensure the VCO had stabilized and the bias voltage at the gate of $\mathrm{M} 7$, Vbias, was varied from $834 \mathrm{mV}$ to $858 \mathrm{mV}$ in $2 \mathrm{mV}$ steps. The calculator frequency function was used to calculate the frequency. The results are plotted in Figure 3.9 , and it is observed that the voltage variation at the bias node does have effect on the output frequency. As can be seen from Figure 3.9 the bias node can vary by approximately $3 \mathrm{mV}$ before the VCO frequency varies from approximately $1.41 \mathrm{GHz}$. 

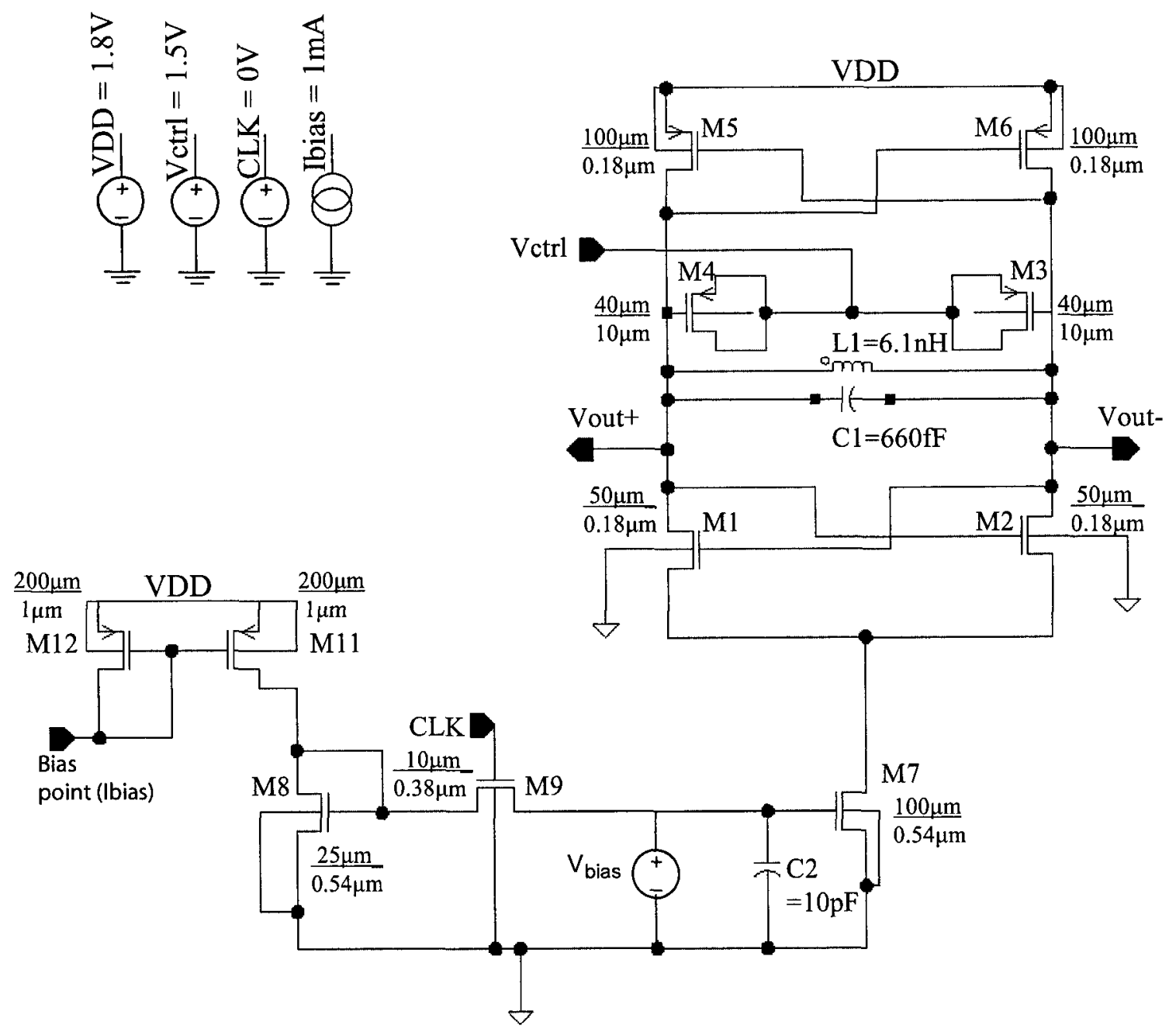

Figure 3.8: Test setup used to model the effect of voltage variation, at the bias node at the gate of M7, on the frequency of the VCO. The voltage, Vbias, at the gate of M7 was swept from $834 \mathrm{mV}$ to $858 \mathrm{mV}$ in $2 \mathrm{mV}$ steps. 


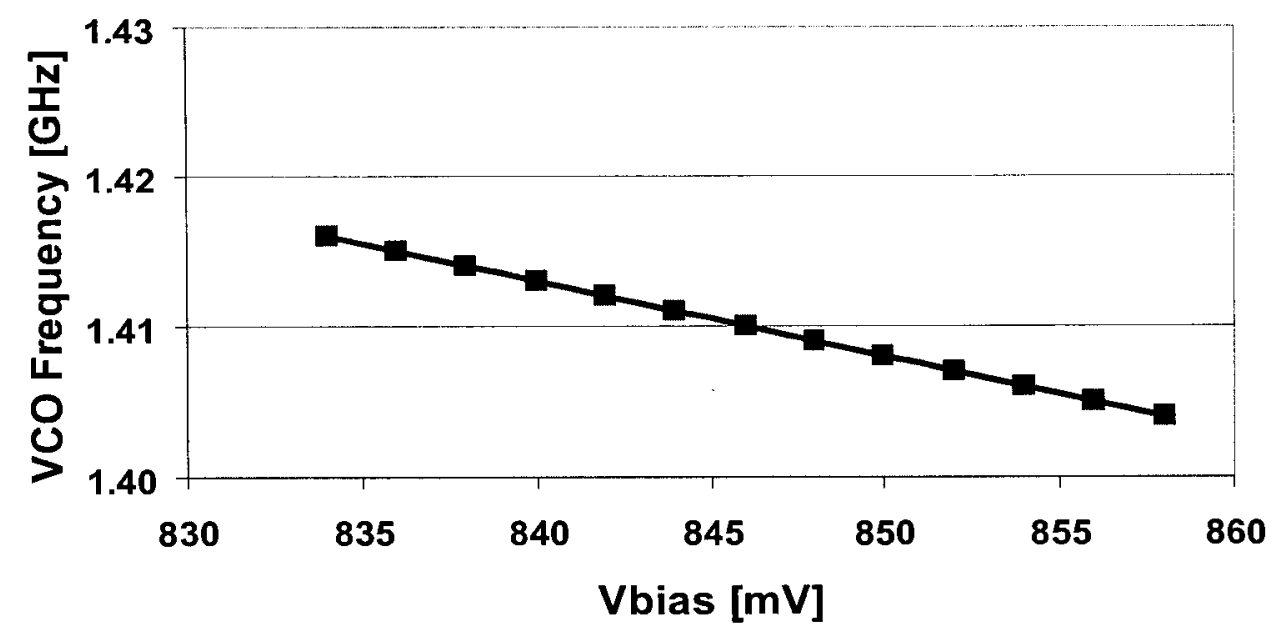

Figure 3.9: Plot of frequency versus bias voltage. This plot was used to determine the effect variation of the bias voltage has on the frequency at the output of the VCO.

\subsubsection{Sizing of Current Mirror}

The noise scaling from the bias network is related to the relative size of the current mirror devices. When the current is ratioed up, the noise is also ratioed up. Low power devices may use current mirror scaling as it allows for a reference current that can be many times smaller than the VCO bias current, helping to reduce the current required overall by the circuit, but resulting in a large multiplying factor for the current mirror. As the device consumes less current using this approach it is able to save power. However, the large multiplying factor leads to a large amplification of the bias network noise.

Therefore the sizing of the current mirror that is being disconnected is another important design consideration. The more amplification there is between the two sides of the biasing network the more the noise will be amplified. Therefore this technique is especially beneficial in the cases were there is a large multiplication factor between the two sides of the current mirror. In the VCO being tested, the multiplication factor is 4 , with a current of $1 \mathrm{~mA}$ being converted to $4 \mathrm{~mA}$ at the base of the $\mathrm{VCO}$, through a 1:4 current mirror ratioing of the sizes of the NMOS transistors. 


\subsection{Application Analysis}

\subsubsection{Applied to a VCO}

The switched-bias and bias retention circuitry were applied to a VCO to show how this technique can be integrated into an application.

\subsubsection{Analysis}

In the case of the $\mathrm{VCO}$, it is the bias network that is being targeted by the switched-bias circuitry to help reduce the overall phase noise. One issue to explore is how the noise on one side of the bias network transfers to the other side of the network, and then into the main circuit, affecting the phase noise the VCO experiences. The transfer function of the noise in the bias network was determined in order to examine this relationship.

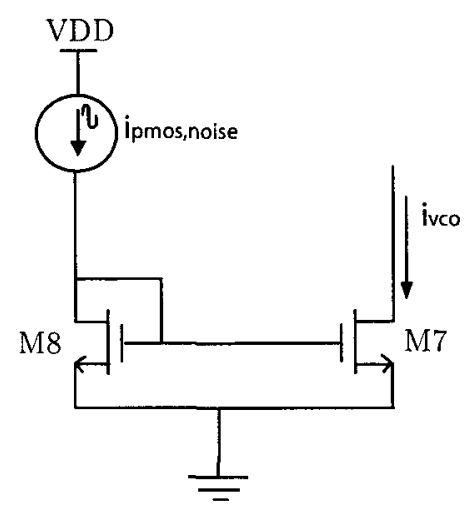

Figure 3.10: Current mirror schematic used to find the transfer function of the bias network.

A schematic of the current mirror is shown in Figure 3.10. While the signals associated with a VCO are generally considered large signal, the noise signal can be considered a small signal, therefore the small signal model shown in Figure 3.11 can be used to find the small signal $i_{v c o}$ as a function of the small signal $i_{p m o s, n o i s e}$. Kirchoff's current law (KCL) can be applied to the small signal model. There are two unknown voltages, which are labelled by " $\mathrm{x}$ " and " $\mathrm{y}$ " on the diagram. KCL is applied at node " $\mathrm{x}$ ", node " $\mathrm{y}$ ", and at the supernode, as is shown in Figure 3.11. This results in the following set of equations, (3.4.1) - (3.4.3), which can be solved to determine the transfer function for $i_{\text {vco }} / i_{\text {pmos,noise }}$. 


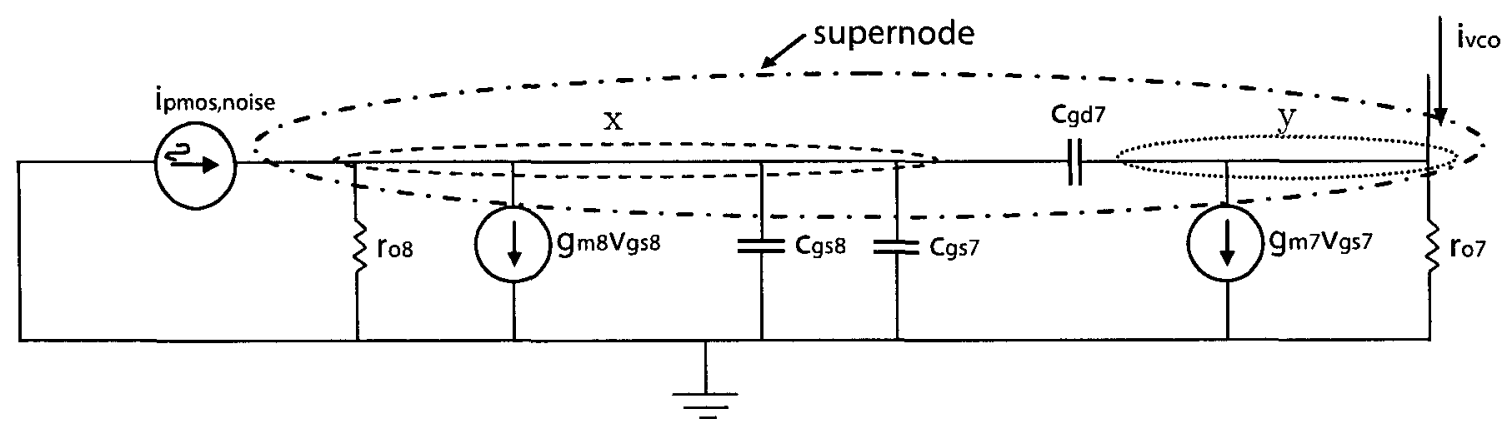

Figure 3.11: The small signal current mirror schematic used to find the transfer function (used to find the small signal $i_{v c o}$ as a function of the small signal $i_{p m o s, n o i s e}$ ) of the bias network.

$$
\begin{gathered}
i_{p m o s, n o i s e}=\frac{v_{x}}{r_{o 8}}+g_{m 8} v_{x}+s\left(c_{g s 8}+c_{g s 7}\right) v_{x}+s c_{g d 7}\left(v_{x}-v_{y}\right) \\
i_{v c o}=\frac{v_{y}}{r_{o 7}}+g_{m 7} v_{x}+s c_{g d 7}\left(v_{y}-v_{x}\right) \\
i_{p m o s, n o i s e}+i_{v c o}=\frac{v_{x}}{r_{o 8}}+g_{m 8} v_{x}+s\left(c_{g s 8}+c_{g s 7}\right) v_{x}+\frac{v_{y}}{r_{o 7}}+g_{m 7} v_{x}
\end{gathered}
$$

The next step is to eliminate $\mathrm{v}_{x}$ and $\mathrm{v}_{y}$ from (3.4.1) - (3.4.3) and then solve for $\mathrm{i}_{v c o} / \mathrm{i}_{\text {pmos,noise }}$. The final result for the transfer function is given by,

$$
\frac{i_{v c o}}{i_{p m o s, n o i s e}}=\frac{r_{o 8}\left(g_{m 7}-s c_{g d 7}\right)}{1+g_{m 8} r_{o 8}+\left(c_{g d 7}+c_{g s 7}+c_{g s 8}\right) r_{o 8} s}
$$

where $\mathrm{g}_{m}$ is the transconductance of the MOSFETs, M7 and M8, which is combined with the relevant $\mathrm{v}_{g s}$ (the gate-source voltage) to form the current source shown, $\mathrm{r}_{o 7}$ and $\mathrm{r}_{o 8}$ are the output resistances of the MOSFETs, M7 and M8, and $\mathrm{c}_{g s}$ and $\mathrm{c}_{g d}$ are parasitic (gate-source and gate-drain, respectively) capacitances associated with the MOSFETs.

Transient simulations were done to confirm the transfer function. The testbench for the simulations is shown in Figure 3.12. A $100 \mu \mathrm{A}$ AC current source with a variable frequency, 


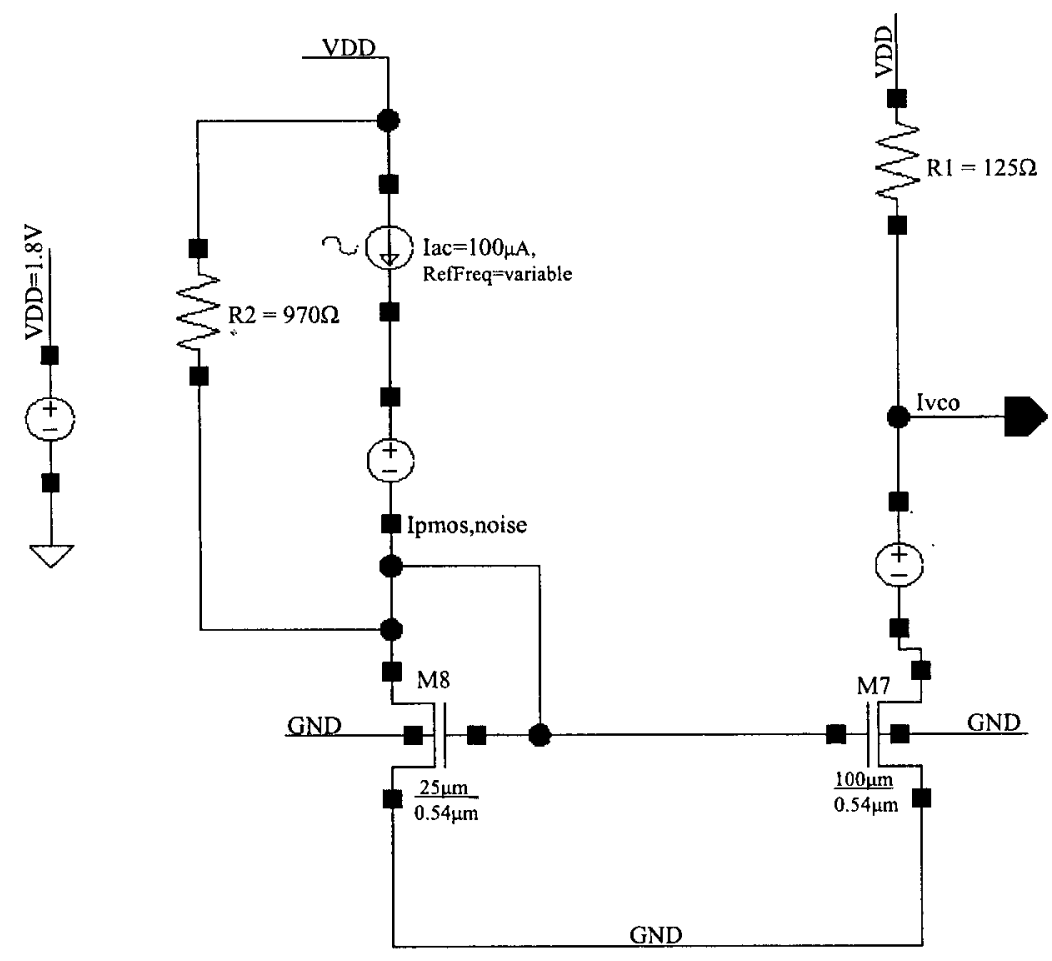

Figure 3.12: Testbench used to simulate the transfer function using transient simulations.

Table 3.1: Transfer function small signal parameter values

\begin{tabular}{|c||c|}
\hline Parameter & Value \\
\hline $\mathrm{g}_{d s 8}$ & $52.88 \mu \mathrm{S}$ \\
\hline $\mathrm{r}_{o 8}$ & $18.91 \mathrm{k} \Omega$ \\
\hline $\mathrm{g}_{m 8}$ & $0.0048 \mathrm{~A} / \mathrm{V}$ \\
\hline $\mathrm{c}_{g s 8}$ & $85.62 \mathrm{fF}$ \\
\hline $\mathrm{g}_{m 7}$ & $0.019 \mathrm{~A} / \mathrm{V}$ \\
\hline $\mathrm{c}_{g s 7}$ & $365.8 \mathrm{fF}$ \\
\hline $\mathrm{c}_{g d 7}$ & $34.29 \mathrm{fF}$ \\
\hline frequency,f & RefFreq (swept value) \\
\hline
\end{tabular}



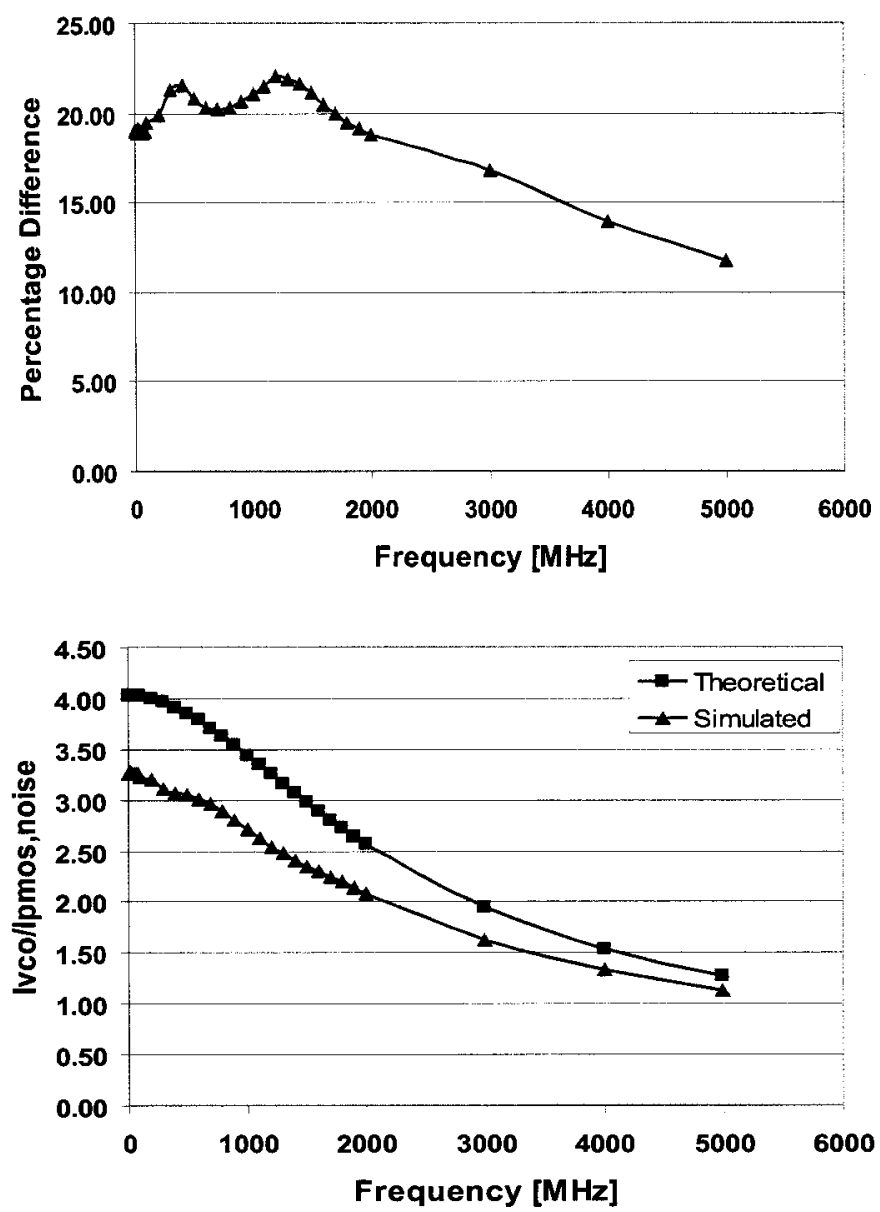

Figure 3.13: Plot of simulated and calculated values for the transfer function given in Equation (3.4.4). The top plot shows the percentage difference between the simulated and calculated results. The bottom plot shows the calculated and simulated transfer function values verses the ac signal frequency, RefFreq. 
RefFreq was used to model the current noise source. Resistors, R2 and R1, were used to bias the current mirror. The calculator function was used to find the amplitude of the signals, Ivco and Ipmos,noise. The amplitudes of these signals were used to determine the transfer function, Ivco/Ipmos,noise. The results from the simulation were then compared against the calculated values for the same range of frequencies and are shown in Figure 3.13. The calculated values were obtained by using the parameters for M7 and M8 that are shown in Table 3.1, in combination with Equation (3.4.4). The simulated and calculated curves have the same shape, though, for the lower frequencies there is a $19 \%$ difference in the values. For lower frequencies, the transfer function indicates that the noise transferred through the current mirror has a greater effect than a higher frequencies. This again agrees with the decision mentioned in subsection 2.3.1 to focus investigating this technique for close-in frequencies.

\subsubsection{Noise Contribution Simulation}

To try and understand the main sources of noise in the VCO, it was required to look at the noise contribution of each MOSFET in the circuit. The MOSFETs contribute noise which ultimately results in the phase noise for the VCO, and therefore it is desired to try to locate and minimize the largest noise sources within the circuit. The noise contribution simulation was done by considering the top 20 noise sources as the total noise was integrated over a bandwidth from $1 \mathrm{kHz}$ to $100 \mathrm{kHz}$. This bandwidth was used because it is the close-in phase noise that was being concentrated on in this research. The parameters for the PSS/Pnoise simulations done using Cadence's SpectreRF simulator in this section are shown in Table 3.2 and Table 3.3.

Table 3.2: PSS Simulation Parameters
\begin{tabular}{|c|c|}
\hline Parameter & Value \\
\hline Beat Frequency & $1.41 \mathrm{GHz}$ \\
\hline Number of harmonics & 10 \\
\hline Oscillator node & Vout+ \\
\hline Reference node & Vout- \\
\hline
\end{tabular}


Table 3.3: Pnoise Simulation Parameters

\begin{tabular}{|c||c|}
\hline Parameter & Value \\
\hline Sweeptype & relative \\
\hline Relative harmonic & 1 \\
\hline Frequency sweep range & Start: $1 \mathrm{kHz}$, Stop: $100 \mathrm{MHz}$ \\
\hline Sweep type & Logarithmic, 20 points per decade \\
\hline Maximum sideband & 10 \\
\hline Voltage out & $\begin{array}{l}\text { Positive Output Node: Vout+ } \\
\text { Negative Output Node: Vout- }\end{array}$ \\
\hline Noise type & sources \\
\hline
\end{tabular}

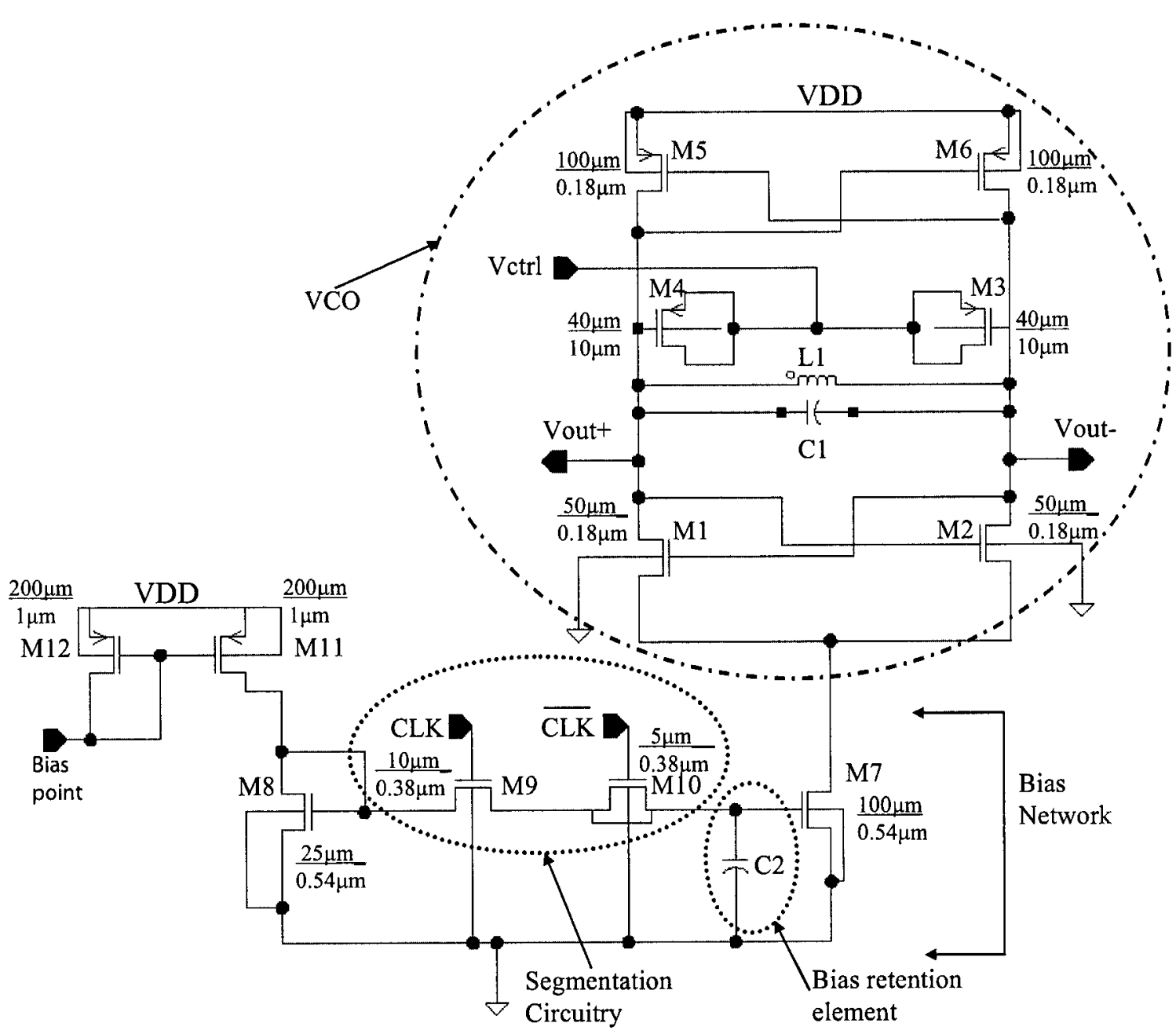

Figure 3.14: VCO circuit with the switched-bias circuitry. The VCO uses a cross-coupled differential inductance-capacitance topology, which is also known as an LC oscillator. 


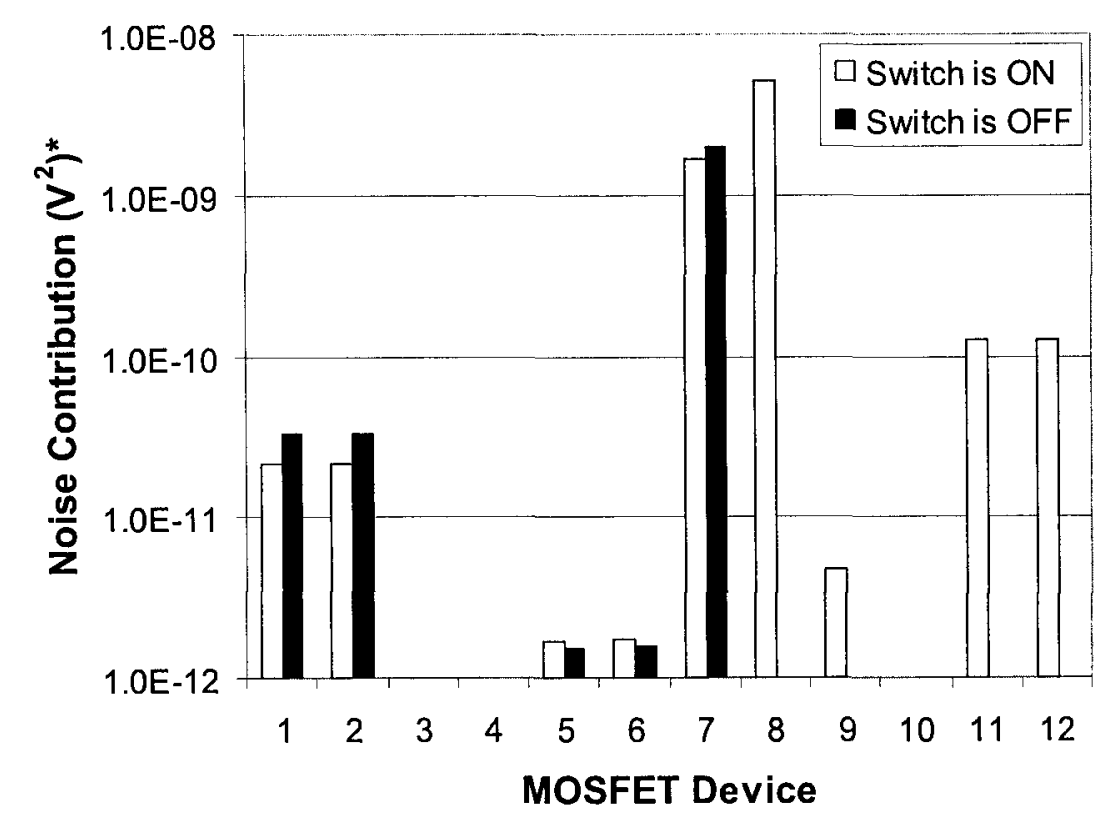

Figure 3.15: Graph of the noise contribution made to the overall integrated output noise, as calculated by SpectreRF over a bandwidth from $1 \mathrm{kHz}$ to $100 \mathrm{kHz}$, by each transistor in the VCO and bias circuit for the case when the switch is turned on and when the switch is turned off. The transistors forming the bias circuitry, M7, M8, M11 and M12, contribute the largest amount of noise when the switch is turned on. M7 is the largest noise source when the switch is turned off. 
To further investigate the role of the bias current, noise simulations were run to observe the various noise contributors in the circuit. Simulations were done for both the case when the switch was on and when it was turned off. The noise contribution generated by each transistor in the circuit was studied, and the results are shown in Figure 3.15. These results show that while the switch is turned on, the bias circuitry, formed by M7, M8, M11 and M12 (see Figure 3.14 for reference) result in the largest noise contribution to the overall circuit. For this case the total integrated output noise as calculated by SpectreRF over a bandwidth from $1 \mathrm{kHz}$ to $100 \mathrm{kHz}$ is $7 \mathrm{nV}^{2}$. When the switch is opened, the bias network becomes disconnected from the VCO circuitry and no longer contributes noise. Figure 3.15 illustrates that in this case M7 becomes the largest noise source, followed by the differential pair, M1 and M2. In this simulation the total integrated output noise, as calculated by SpectreRF over a bandwidth from $1 \mathrm{kHz}$ to $100 \mathrm{kHz}$, is $2 \mathrm{nV}^{2}$.

From the results in Figure 3.15, and the change in the total integrated output noise, it is seen that the bias circuitry in this demonstration circuit does contribute a significant amount of noise within the circuit, and that the switched-bias circuitry is effective in reducing this noise when the switch is opened. The results show that the addition of the switched-bias circuitry does not result in any significant additional noise to the circuit. The noise contributions for many of the transistors are negligible as they are approaching zero and therefore do not appear on the bar graph presented in Figure 3.15.

The transfer function derived in subsection 3.4.2 showed how noise was transferred through the current mirror. The simulation presented in this section have shown that the transistors forming the bias network contribute the largest amount of noise within the circuit. By combining the two results, one can see that using switched-bias circuit to turn off a portion of the bias network will result in a reduction of the overall phase noise seen at the output of the VCO. The simulations verifying this technique will be discussed in the next section, as well as in section 4.5 . 


\subsubsection{Phase Noise Simulations}

The phase noise of the VCO was simulated using SpectreRF to verify the effectiveness of the switched-bias circuitry. The results are summarized in Table 3.4 and Figure 3.16. The simulation parameters used are the same as those that were used to perform the noise contribution simulation as shown in Table 3.2 and Table 3.3. The simulations were run first using a MIM capacitor as the bias retention element, and then using a MOSCAP as the bias retention element. It was observed that the phase noise did not change between the two cases. This is due to the fact that, even though the MOSCAP is formed from an active device, it contributes a negligible amount of noise to the overall integrated output noise as calculated by SpectreRF over a bandwidth from $1 \mathrm{kHz}$ to $100 \mathrm{kHz}$ (on the order of $1.9 \times 10^{-19} \mathrm{~V}^{2}$ ) and therefore does not affect the phase noise value. The advantage of using a MOSCAP versus a MIM capacitor is in terms of the area required. For a $10 \mathrm{pF}$ capacitor, in the $0.18 \mu \mathrm{m}$ CMOS technology, an NMOS MOSCAP requires an area of approximately $2600 \mu \mathrm{m}^{2}$, while, for the same capacitance value, a MIM capacitor requires approximately $11100 \mu \mathrm{m}^{2}$. However, MOSCAPs are non-linear and have a capacitance that is variable and dependent on the bias at the gate, while MIM capacitors have more stable capacitance values as they are independent of input voltage values.

Table 3.4: Phase noise for various states of the switch and offset frequencies

\begin{tabular}{|l||l|c|c|c|}
\hline \multicolumn{1}{|l||}{$\begin{array}{l}\text { Description of } \\
\text { the case }\end{array}$} & \multicolumn{5}{|c|}{$\begin{array}{l}\text { Phase Noise Measurement }[\mathrm{dBc} / \mathrm{Hz}] \\
\text { at various offset frequencies }\end{array}$} \\
\cline { 2 - 5 } & $100 \mathrm{kHz}$ & $200 \mathrm{kHz}$ & $600 \mathrm{kHz}$ & $1 \mathrm{MHz}$ \\
\hline No Switch & -81.48 & -89.9 & -103.1 & -109.1 \\
\hline Switch ON & -81.39 & -89.76 & -102.8 & -108.7 \\
\hline Switch OFF & -87.59 & -96.02 & -109.2 & -115.3 \\
\hline
\end{tabular}

As demonstrated in Table 3.4, the addition of the noise reduction circuitry has minimal impact on the phase noise, with a typical increase in phase noise of approximately $0.3 \mathrm{~dB}$ across the simulated range of frequencies. However, by opening the switch when the output was being measured, the phase noise is reduced across the swept offset frequency range by, on average, $6 \mathrm{~dB}$, which is a noticeable improvement in phase noise. This result matches the expected behaviour of a reduction in phase noise with the temporary removal 
of the bias network from the circuit through the switched-bias circuitry.

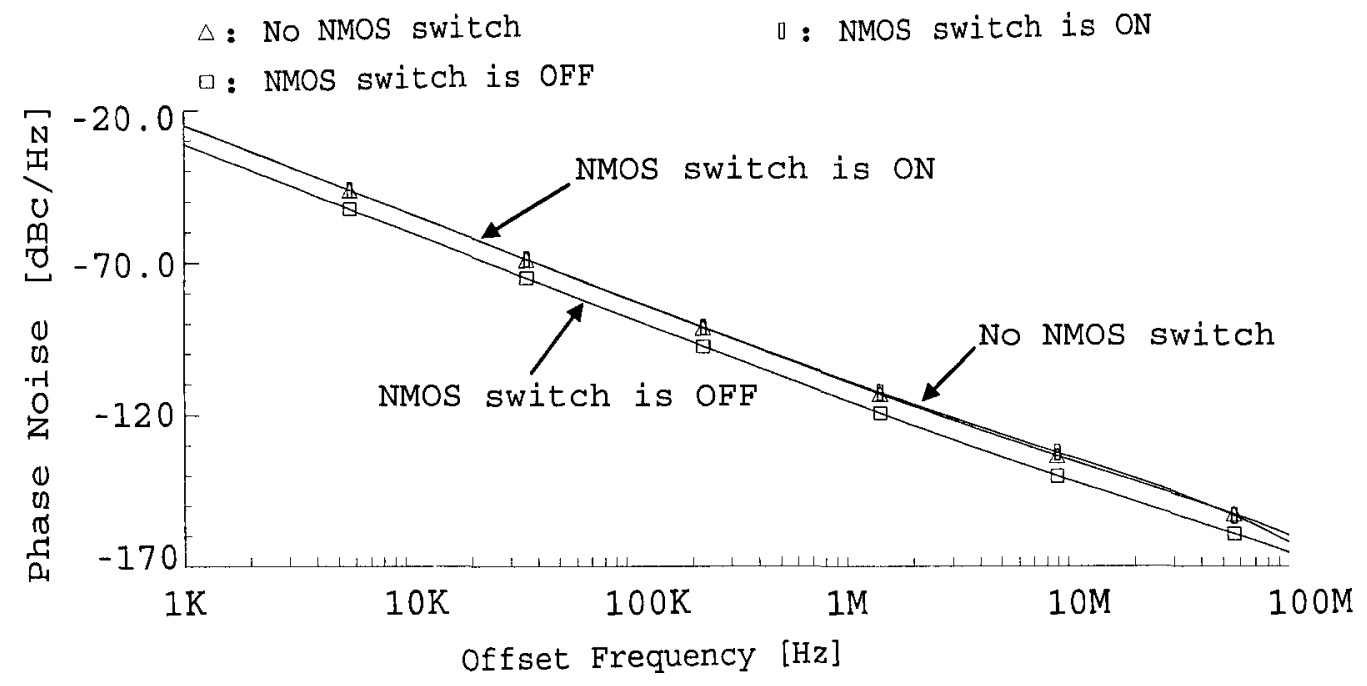

Figure 3.16: Single-sideband (SSB) phase noise simulations over various offset frequencies. The phase noise values for each of the three states of the circuit (no switch, with the switch on and with the switch off) were plotted. This simulation shows the decrease in phase noise when the switch is turned off.

\subsection{Chapter Summary}

- This chapter began with an overview of the switched-bias method and how it was implemented.

- Next the focus shifted to the design issues that were addressed within the circuitry.

- Finally, an analysis of the transfer function of the bias network, noise contribution and VCO phase noise simulations were presented. The design and implementation of $\mathrm{VCO}$ are presented in the next chapter. 


\section{Chapter 4}

\section{Design and Imlpementation}

\subsection{VCO design}

The switched-bias technique was applied to the VCO presented in [8, pp.12-40]. This VCO had been designed and fabricated by another member of the Radio-over-Fibre group at Carleton University, Daniel Olszewski, for a previous project. The full details of the design and implementation of the VCO are presented in [8, pp.12-40], but a summary will be provided here.

The VCO was designed for a radio over fibre receiver. It was designed to generate a local oscillator signal at $800 \mathrm{MHz}, 900 \mathrm{MHz}$ or $1.4 \mathrm{GHz}$, though only the $1.4 \mathrm{GHz}$ implementation is used in this research. The VCO was implemented with the Cadence design suite, using the $0.18 \mu \mathrm{m}$ CMOS technology models provided by CMC.

The cross-coupled differential inductance-capacitance VCO topology, more commonly known as an LC oscillator, presented in [2] was used as the basis for this design. A schematic diagram of the VCO, including the device sizes used, is shown in Figure 4.1. An LC oscillator topology was chosen because, as was discussed in section 2.2 .3 , this VCO generally has lower phase noise then, for example, an ring oscillator.

A complementary structure was chosen over an all-NMOS VCO design, as the complementary structure results in a higher transconductance for a particular current. This higher transconductance then translates to faster switching of the cross-coupled differential pair, which is why larger sizes were chosen for the PMOS and NMOS cross-coupled pair. In addition, as stated in [2] the complementary structure also results in improved rise 


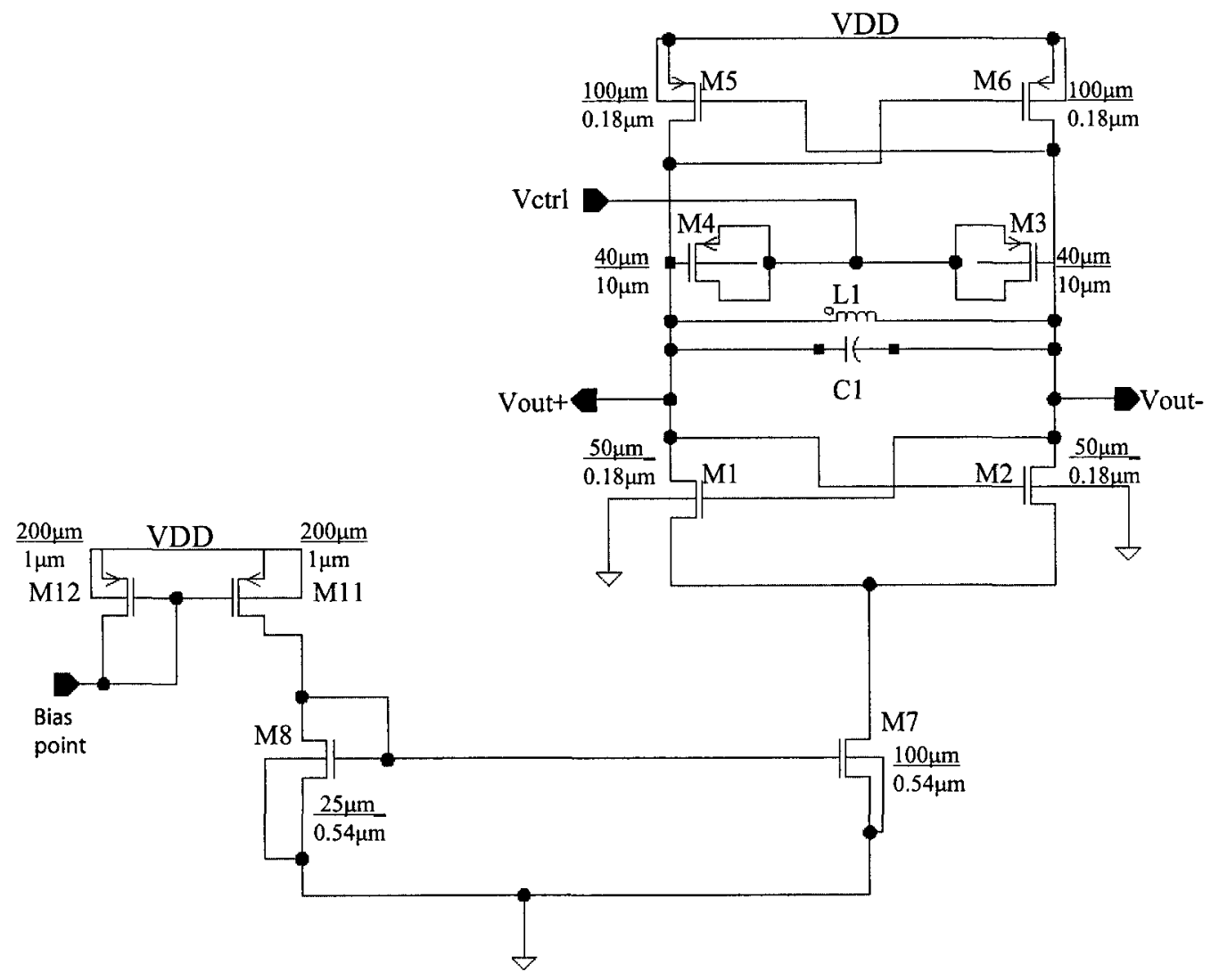

Figure 4.1: VCO circuit only (i.e. it does not contain the switched-bias circuit). The VCO uses a cross-coupled differential inductance-capacitance topology, which is also known as an LC oscillator. 
and fall time symmetry, which in turn results in a smaller $1 / \mathrm{f}^{3}$ noise corner. In addition it was shown in [2] that using the complementary structure allows the circuit to maintain single-ended symmetry for each half of the circuit, which reduces the upconversion of $1 / f$ noise.

The tuning of the VCO is controlled by Vctrl, the control voltage for the MOSCAPs. The differential outputs of the VCO are Vout+ and Vout-. Buffers were added for the fabricated chip to help drive the load at the output due to the test equipment. In addition, in a complete system, these buffers would be used to isolate the VCO from the rest of the circuitry in the system. The buffers provide a way to convert the VCO output from a differential output to the single-ended output that was desired for testing purposes. The power supply, VDD, of the VCO was set to $1.8 \mathrm{~V}$ and the substrate was attached to ground.

The bias network was modified by the addition of the switched-bias circuit designed within this research. The NMOS transistors, M7 and M8, and the PMOS transistors M11 and M12, form the current mirrors that provide the bias current for the VCO. It is shown in [2] that increasing the tail current results in an improvement in phase noise as a result of an increase in the oscillation amplitude. Taking this into account, the bias network was designed to bias the VCO with a constant $4 \mathrm{~mA}$ current. This provides a balance between using a larger current to help reduce phase noise, while still being conscious of the power consumption of the circuit. In addition, larger transistor sizes were used to ensure that the transistors in the current mirror remained in saturation. The lengths of the transistors in the current mirror were made longer than the minimum length required, in part to help lower the channel length modulation effects $(\lambda)$. Following the design guidelines presented in [35, pp.228], to reduce the effects of channel length modulation, "the length of MOSFETs used in analog applications should be set to two to five times the minimum gate length." The larger sizes of the transistors results in an increased output resistance of the current mirror, which helps to make the bias current less dependent on the drain to source voltage of the transistor. And lastly, as it has already been discussed in 2.3.2, and shown in [41], the flicker noise of a MOS transistor is inversely proportional to the gate 
area. Therefore the longer lengths of the transistors provide a larger total area, which helps to improve the phase noise of the VCO by reducing the flicker noise associated with the MOSFETs forming the current mirror.

The LC tank for the VCO comprises of an on-chip 6.1nH spiral inductor and an integrated $660 \mathrm{fF}$ fixed capacitor, in parallel with PMOS MOSCAPs, which were using for tuning. The model of the inductor, which was provided by $\mathrm{CMC}$ in [42], is shown in 4.2. The fixed capacitor was implemented using the parallel plate metal-insulator-metal (MIM) capacitor found in the CMC $0.18 \mu \mathrm{m}$ design library. The quality factor, $\mathrm{Q}$, of the tank is 9.9 for the VCO when the center frequency is $1.4 \mathrm{GHz}$. The inductor was designed using the guidelines provided in [43, pp.223-235] which states that "inner turns of the onchip spiral inductor should be removed because they contribute little to the inductance, but add to the parasitic losses experience by the inductor," and that, "the upper layers of metal should be used to reduce parasitic capacitances to ground."

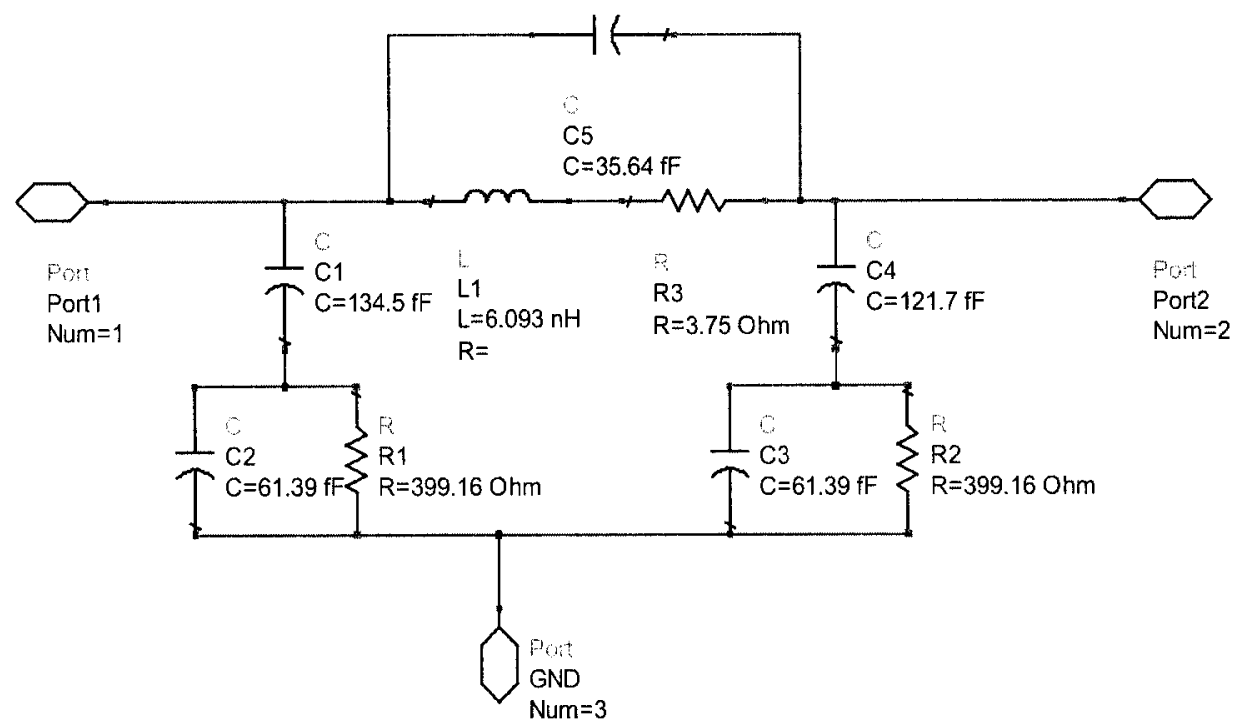

Figure 4.2: Model used to simulate the inductor.

Table 4.1 provides a summary of the main simulated results, especially phase noise, which is of particular relevance to this thesis. The VCO presented [8, pp.12-40] provided a reference point to use in this thesis for the case of the VCO without the switched-bias 
circuitry which would not have otherwise been possible due to space limitations associated with the fabrication process.

Table 4.1: Summary of simulation results for the cross-coupled LC VCO

\begin{tabular}{|c||c|}
\hline Center Frequency & $1.41 \mathrm{GHz}$ \\
\hline VCO Gain & $400 \mathrm{MHz} / \mathrm{V}$ \\
\hline Peak Differential Output Swing & $1.43 \mathrm{~V}$ \\
\hline Phase noise (at 1MHz offset from carrier) & $-109.2 \mathrm{dBc} / \mathrm{Hz}$ \\
\hline DC Power Dissipation (from 1.8V supply) & $6.6 \mathrm{~mW}$ \\
\hline Tuning range & $17 \%$ \\
\hline
\end{tabular}

The VCO without the switched-bias circuit was tested and the measured characteristics are shown in more detail in Section 5.2.2. The main purpose of using this particular VCO was that it had been previously fabricated and could therefore serve as a benchmark against which the VCO containing the switched-bias noise reduction circuitry could be compared.

The phase noise measurement results for the VCO using its center control voltage of $1.5 \mathrm{~V}$ are shown in Table 4.2 , while the simulated and measured values were plotted together, as can be seen in Figure 4.3. The measurement results will be discussed in more detail in Section 5.2.2.

Table 4.2: Phase Noise Measurements

\begin{tabular}{|c||c|c|}
\hline Spot Freq. $[\mathrm{kHz}]$ & Carrier Freq. [GHz] & SSB phase noise $[\mathrm{dBc} / \mathrm{Hz}]$ \\
\hline 100 & 1.47041 & -87.4 \\
\hline 200 & 1.47041 & -77.54 \\
\hline 600 & 1.47040 & -97.3 \\
\hline 1000 & 1.47042 & -102.3 \\
\hline
\end{tabular}

\subsection{Integration of switched-bias circuit into the VCO}

As mentioned previously the VCO has been designed in [8, pp.12-40], and the task was to integrated the switched-bias circuitry into this VCO design. This integration was done by cutting the bias circuitry into two sections, as is shown in Figure 3.14 and Figure 4.4, and inserting the switched-bias and bias retention circuitry at the point where the cut was 


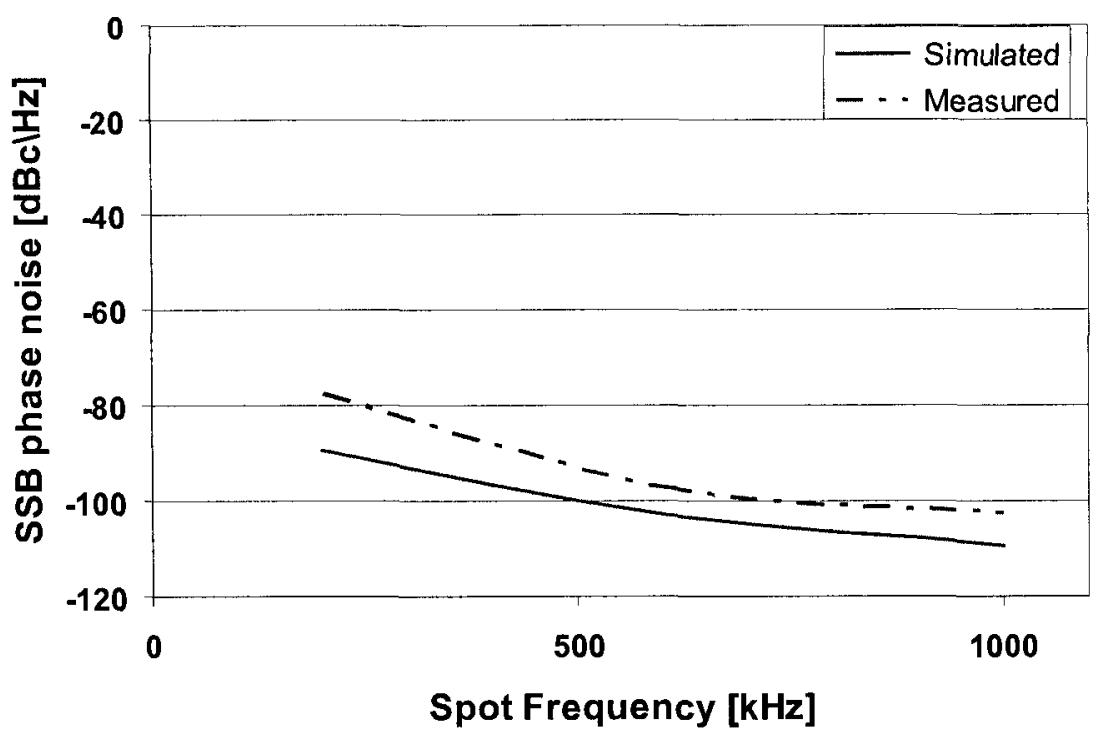

Figure 4.3: The measured and simulated phase noise results for the VCO.

made. This configuration provides a method to disconnect Bias A, as shown in Figure 4.4, from the overall circuit, allowing the noise associated with that portion of the bias network to be removed from the overall phase noise of the VCO. Details of the switched-bias circuit design were already presented in section 3.1 to section 3.4.1, and the VCO containing the bias network with the switched-bias circuit incorporated into it was shown in Figure 3.14.

The actual implementation of the switched-bias circuit that was fabricated included two switches (and two dummy switches). The reason this was done was to allow experimentation with the resistance value of the switch by allowing some flexibility by placing the two resistors in parallel by turning them both on and off at the same rate, as well as testing the circuit with a single set of switches (i.e. a single main and dummy switch). The size of both sets of main switches and dummy switches are equal and are equal to the switches shown by M9 and M10, respectively in Figure 3.14. Another modification to the switched-bias circuit that was made to facilitate testing was the addition of a signal named DischargeIn. This signal was attached to the gate of an NMOS transistor and provided a mechanism to discharge the bias retention capacitor used within the switched-bias circuit 


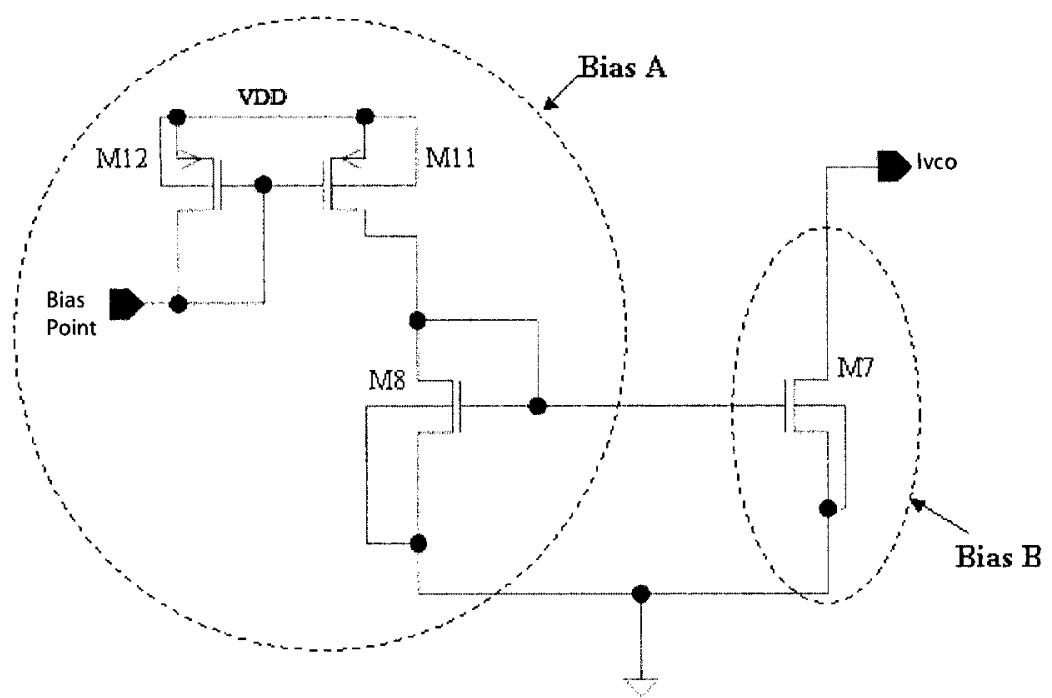

Figure 4.4: Biasing network. It has been divided into two sections, Bias $\mathrm{A}$, which is disconnected from the VCO by the switch, and Bias B, which is continually connected to VCO circuit.

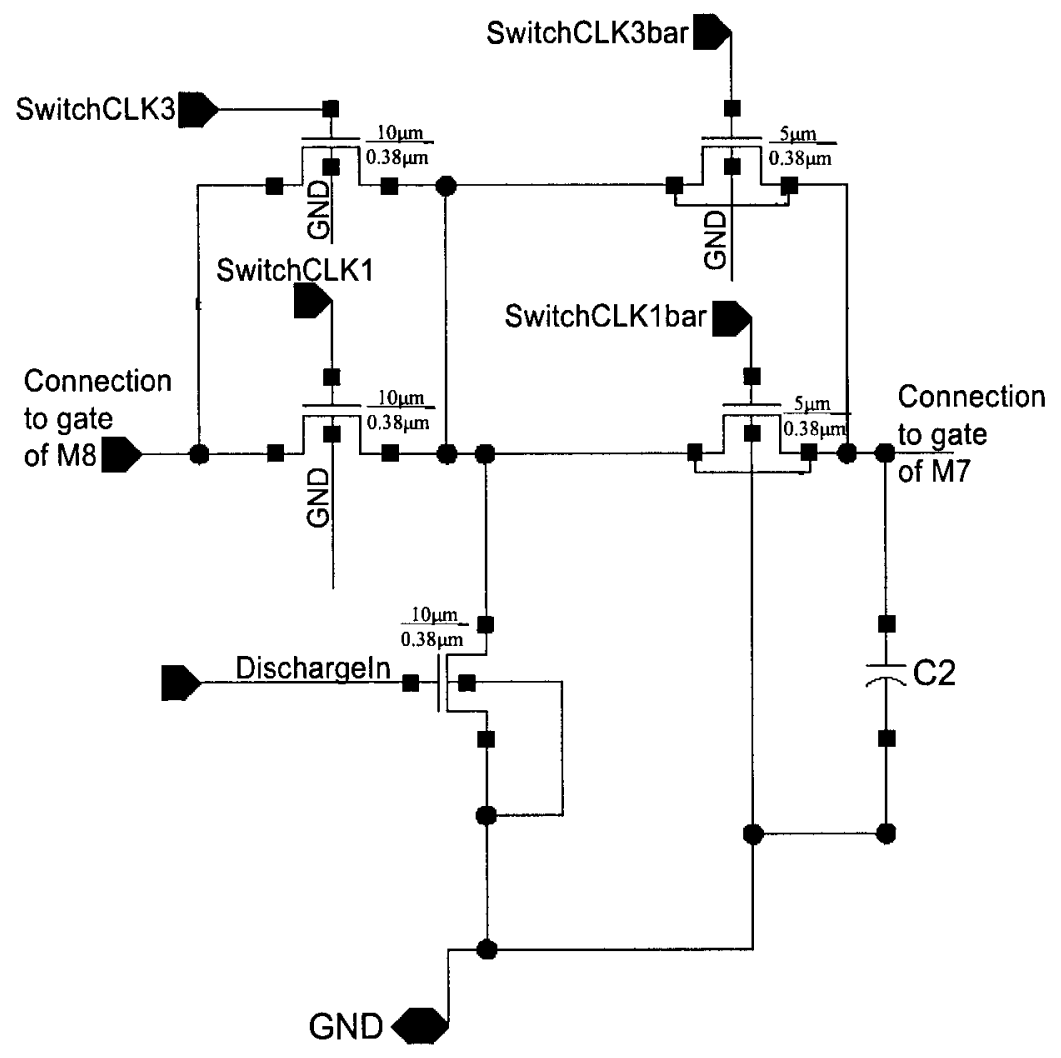

Figure 4.5: Switched-bias circuit including circuitry added to facilitate testing. 
(C2). The NMOS transistor has its drain attached to the the capacitor node, and its source attached to ground, and its size was made the same as the main switch. This extra transistor does not form part of the switched-bias circuit and is used to facilitate testing by providing a way to ensure the capacitor does not start with charge already built up on the node before the switch is closed, as this would effect the time measured for which the capacitor can hold the bias value at a constant voltage. The actual switched-bias circuit that was fabricated, including these test structure is shown in Figure 4.5.

\subsection{Layout Considerations}

The complete circuit was implemented in the $0.18 \mu \mathrm{m}$ CMOS process available from the Taiwanese Semiconductor Manufacturing Corporation (TSMC), through CMC Microsystems. This is a six metal layer CMOS process with a single salicided poly layer. It uses a p-type substrate doping, and as a result all PMOS devices are formed in $\mathrm{N}$-wells. On this chip, all of the NMOS devices share a common substrate, which is connected to ground, while the PMOS devices are isolated in their own wells to help reduce the body effect. The nominal supply voltage, VDD, is $1.8 \mathrm{~V}$. The layout was done using the Cadence Design Virtuoso tool. Several layout considerations were taken into account while doing the layout, and they will be discussed for the remainder of this section.

The layout used in [8, pp.37-39] was modified to include the switched-bias circuit. The layout, with the pad structure, is shown in Figure 4.6. An explanation of the pads given in Table 4.3. The layout includes the VCO, which uses MOSCAPs for tuning, a MIM capacitor and a rectangular spiral inductor for the tank, and has an internal VCO output buffer. There is an additional output buffer to convert the differential signals to single-ended, and to allow the VCO to drive the bond-wires and capacitive loads.

To help reduce the effects of process variations, the gates of all of the transistors were broken into a number of parallel fingers, with dummy gates at the ends. Guard rings, which involved surrounding the transistors by substrate contacts ( $\mathrm{p}$-substrate for NMOS transistors and $\mathrm{n}$-substrate for PMOS transistors), were used to help isolate the transistors and prevent coupling and noise injection from other devices sharing the same substrate. 


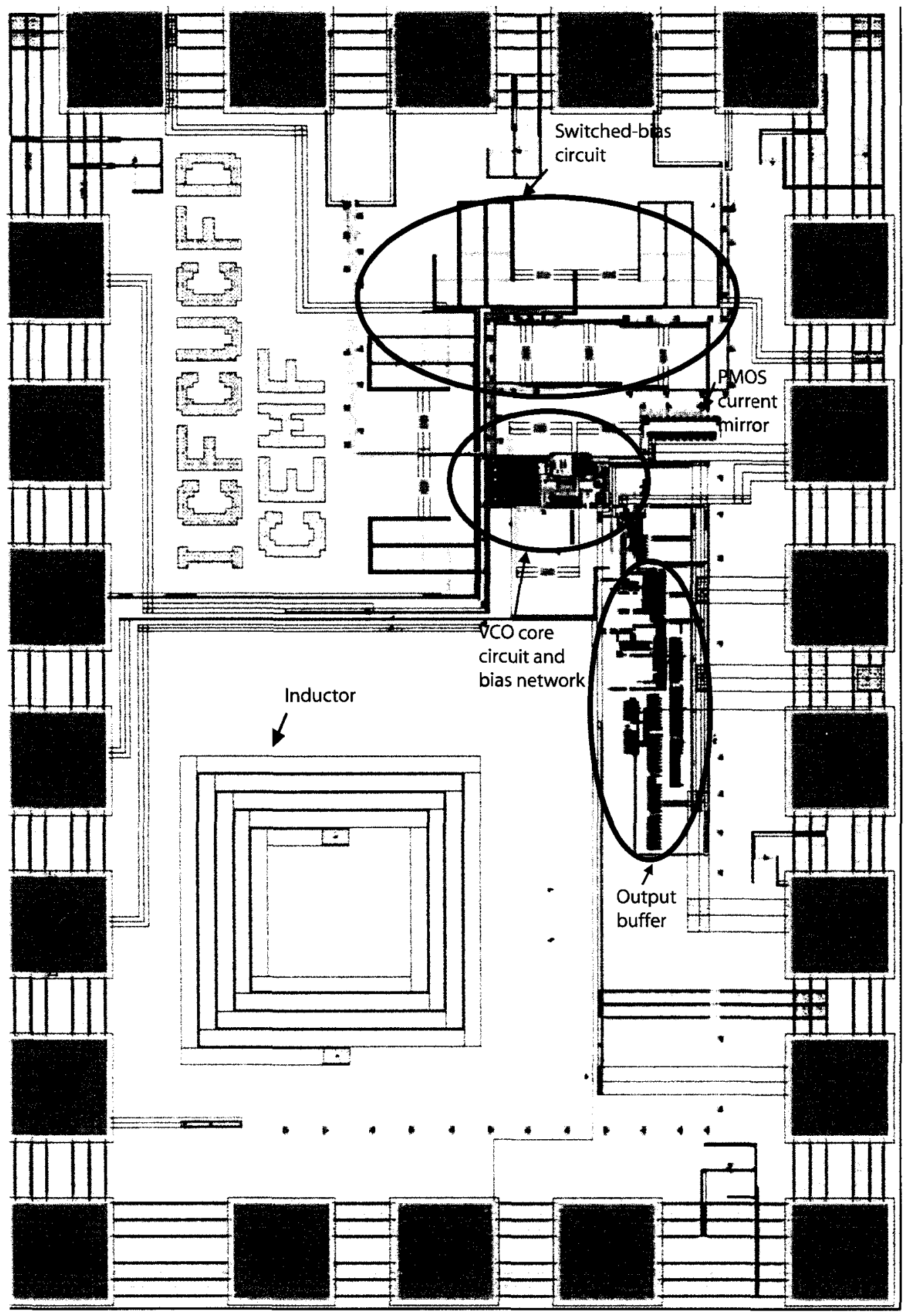

Figure 4.6: Placement of the VCO circuit including the switched-bias circuitry. 
Table 4.3: List of the pad names used for the placement of the VCO

\begin{tabular}{|c|c|c|}
\hline Number & Pad Name & Explanation \\
\hline $1,8,10,12,13,15,20$ & GND & Ground \\
\hline 2 & SwitchCLK3bar & $\begin{array}{l}\text { Second clock input used to control } \\
\text { the second dummy switch }\end{array}$ \\
\hline 3 & SwitchCLK1bar & $\begin{array}{l}\text { First clock input used to control } \\
\text { the first dummy switch }\end{array}$ \\
\hline 4 & SwitchCLK1 & $\begin{array}{l}\text { First clock input used to control } \\
\text { the first main switch }\end{array}$ \\
\hline 5 & SwitchCLK3 & $\begin{array}{l}\text { Second clock input used to control } \\
\text { the second main switch }\end{array}$ \\
\hline $6,7,11,16,21$ & VDD & Power Supply (1.8V) \\
\hline 9 & Fout & Output of the VCO \\
\hline 14 & VcapTest & $\begin{array}{l}\text { Test point at the gate of M7 in } \\
\text { the bias network }\end{array}$ \\
\hline 17 & Ibias & $\begin{array}{l}\text { Bias current }(1 \mathrm{~mA}) \text { attached to } \\
\text { the PMOS current mirror }\end{array}$ \\
\hline 18 & DischargeIn & $\begin{array}{l}\text { Input to an NMOS used to discharge } \\
\text { the bias retention capacitor during testing }\end{array}$ \\
\hline 19 & IoutBuf & $\begin{array}{l}\text { Input current }(240 \mu \mathrm{A}) \text { to } \\
\text { the buffer circuitry }\end{array}$ \\
\hline 22 & Vctrl & $\begin{array}{c}\text { Control voltage applied to the MOSCAPs } \\
\text { MOSCAPS used to tune the VCO }\end{array}$ \\
\hline
\end{tabular}


$4.3 \mu \mathrm{m}$ wide metal lines were used for VDD and GND, and in general the metal lines used to connect devices were made wider then required by the minimum design rules to ensure that they could handle the required current for the circuit. In addition, multiple parallel vias and contacts were used between layers to help improve reliability as well as to decrease the parasitic resistance between layers.

To use the least area possible, the minimum design rules required for spacing between devices as enforced by Cadence Design Virtuoso tool were used. The exception to this practice was the spacing around the inductor. The largest possible distance between the inductor and the rest of the circuitry was used to reduce losses experience by the inductor in terms of its $\mathrm{Q}$ value which may result due to interference to the magnetic field from the rest of the circuit. In addition, the inductor was built using the upper layer metal to help reduce parasitic capacitances to ground which can also reduce the $\mathrm{Q}$ value of the inductor.

The power supply (VDD) and ground (GND) signals were placed in a ring structure around the perimeter of the chip, with multiple VDD and GND pads used to help improve reliability. In total there are seven ground pads, and five power supply pads. The reason there are so many VDD and GND pads is not only to improve reliability, in terms of ensuring a good ground and power supply connection, but also to reduce supply-bounce that is caused by bond-wire inductance, by helping to reduce the overall equivalent inductance (due to the pads being connected in parallel). This in turn reduces the corresponding $\mathrm{L} \cdot \frac{d I}{d t}$ effect. Though supply-bounce is not a major concern in an LC oscillator because ideally the bias current (i.e. the current through M7) should remain constant as the VCO switches back and forth. However, the fabricated chip will not be ideal, so precautions were taken by added extra bond-wires. Transient simulations were done to ensure that the addition of the bond-wires did not negatively impact the VCO's frequency. The high frequency output, $\mathrm{F}_{\text {out }}$, metal trace was made as short as possible (128 $\mu \mathrm{m}$ in length) when connecting it to the pad to help reduce the effects of parasitic capacitance and resistance on this node. 
To help reduce supply and substrate noise, five decoupling MIM (metal-insulatormetal) capacitors (with values of $1-2 \mathrm{pF}$ ) were placed on chip between VDD and GND. All of the MIM capacitor layouts used in this design (including the bias retention capacitor) were generated using the "spccap" parameterized cell provided by CMC in the CMCpcells folder.

A photograph of the fabricated chip, referred to as ICFCUCFD, is shown in Figure 4.7. The overall dimension of the design is $819 \mu \mathrm{m}$ by $1193 \mu \mathrm{m}$. Twenty-two bonding pads are used in a $1.2 \mathrm{mmx} 0.82 \mathrm{~mm}$ rectangle. The bond-pads are square with a size of $100 \mu \mathrm{m}$ by $100 \mu \mathrm{m}$, with $50 \mu \mathrm{m}$ spacing $(150 \mu \mathrm{m}$ pitch). The first metal was not used in the pads because the power supply and ground traces were routed under the pad ring due to space restrictions.

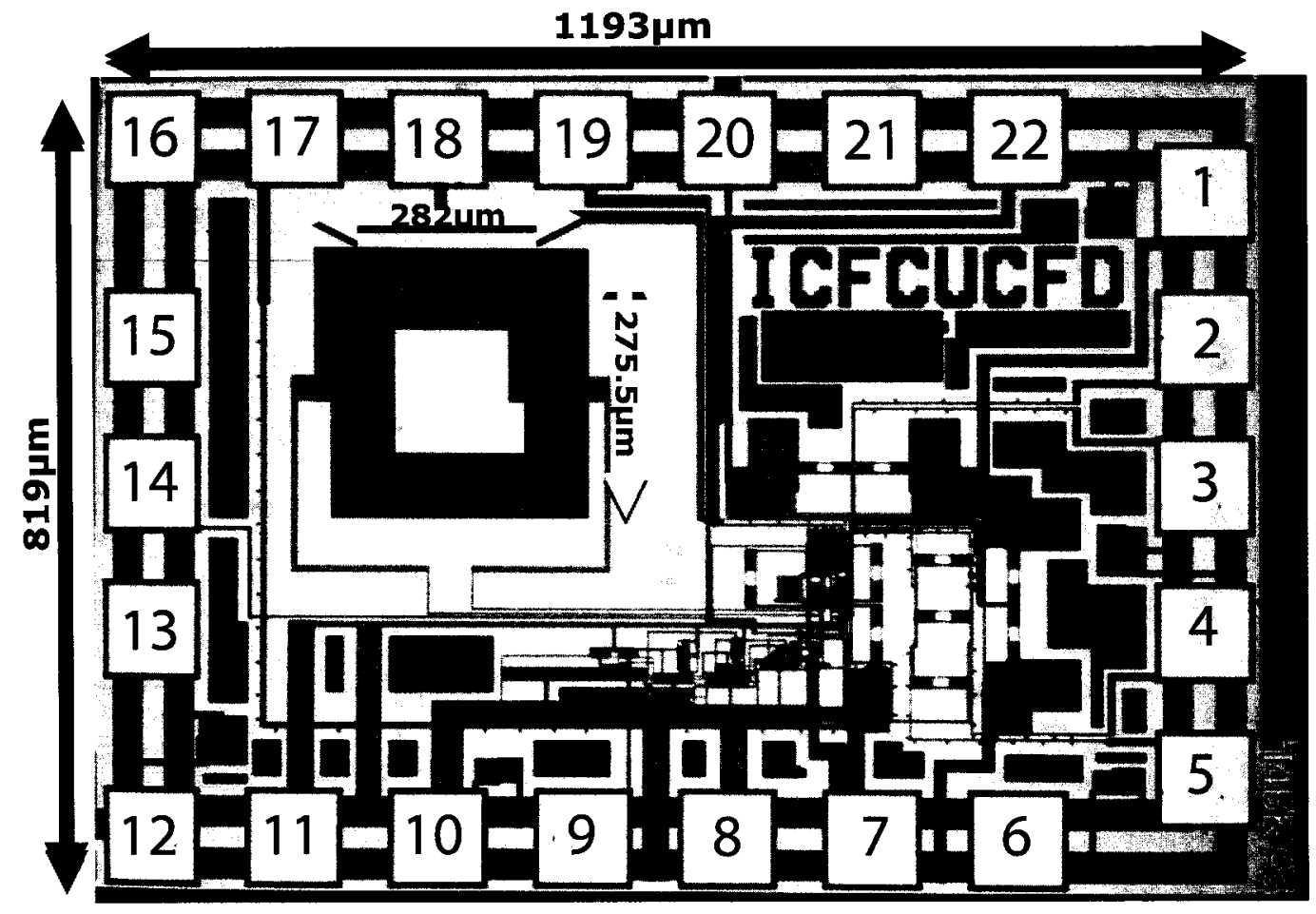

Figure 4.7: A photo of the chip layout. Refer to Table 4.3 for the pad names.

The work of three researchers was placed on the chip space granted by CMC. A photo of the layout for the complete chip is shown in Figure 4.8. Due to the sharing of the space, the placement of the RF output signal was carefully chosen. This signal had to be 
placed on the outer edge of the chip to reduce the length of the bond-wire required. The DC signals, including Vctrl, the bias input currents and the voltage used to discharge the capacitor, were all placed on the inside edge of the chip, as these signals could handle longer bond-wires without degradation to the signals. The clock signal was also placed on an outer edge to help reduce bond-wire length. A test point was added at the bias retention capacitor node to monitor the voltage at this point. This test point could lead to extra capacitance at the gate of M7 in the bias network as a bond-wire is used to attach this point to the PBC used during testing of the chip. However the capacitance of the bond pad was simulated in Cadence by using a layout of a resistor with and without a pad, extracting the layout to obtain the parasitic capacitances, and finding the bond pad capacitance by subtracting the values. The result of this calculation was a bond pad capacitance of approximately $344 \mathrm{fF}$. As this capacitance value is much smaller then the $10 \mathrm{pF}$ capacitance of the bias retention capacitor to which the test point is connected, it was determined that the test point would not have a negative impact on the circuit, while providing a way to obtain the bias value during the testing process. This test point also provides a way to directly bias the VCO if the current mirror does not work, by providing a mechanism to directly set the voltage at the gate of M7.

\subsection{Cadence Simulations}

Simulations were done to observe the impact the bias network has on the phase noise of a VCO. The expectation was that when a portion of the bias network was disconnected from the circuit, the noise associated with this circuitry would have a reduced impact on the overall phase noise. This would result in a decrease in the VCO's phase noise.

The Cadence Design Suite was first used to simulate the circuit because the layout was being done with Virtuoso, Cadence's layout editor. In Cadence, the phase noise of an oscillator is measured using a periodic steady-state (PSS) simulation, combined with a periodic noise (Pnoise) simulation. A challenge in terms of simulating was encountered while trying to measure phase noise for the VCO containing the switched-bias circuitry. SpectreRF, the simulator that was used in Cadence, considers an oscillator as a purely 


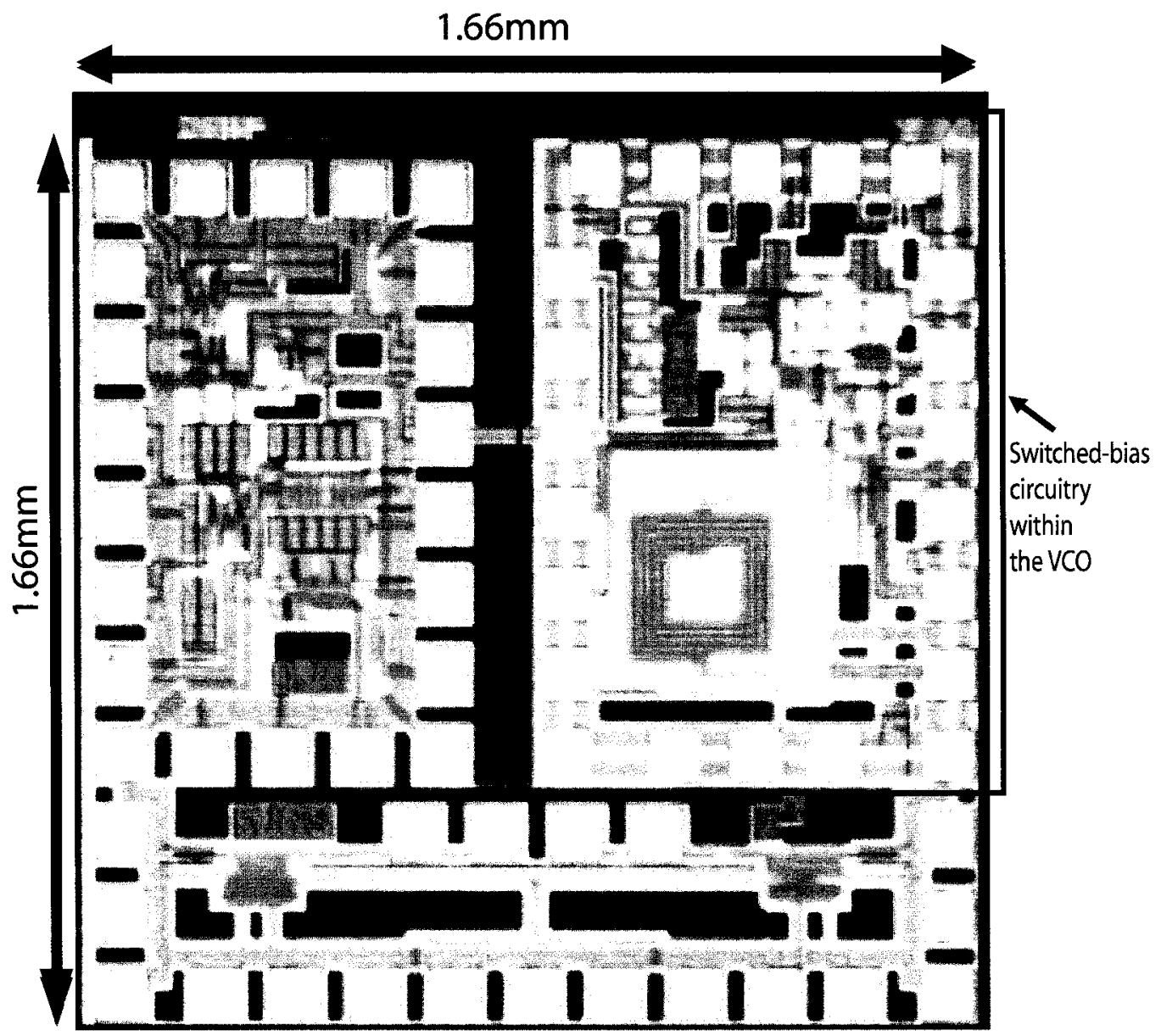

Figure 4.8: A photo of the layout for all three designs included on the fabricated chip. 
autonomous circuit when a phase noise measurement is being performed and therefore did not simulate if the circuit had any inputs. However, while the clock controlling the NMOS switch of the switched-bias circuit is not a direct input to the VCO itself, the simulator is unable to distinguish this fact, resulting in a failed simulation.

Several different strategies were used to work around this problem. It was possible to simulate the VCO if the main switch, M9, (see Figure 3.14) was held static in either the $\mathrm{ON}$ or OFF state for the duration of the simulation. With the main switch in the $\mathrm{ON}$ state, the phase noise could be measured, and matched well with the phase noise for the case of the VCO without the extra switched-bias circuitry. This illustrates that the added circuitry does not introduce an appreciable amount of extra noise. However, when the switch is in the OFF state, while the simulation will run, the phase noise values cannot be considered accurate because the voltage value of the gate of M7 (refer to Figure 3.14, which is at the base of the $\mathrm{VCO}$, does not start at a known value.

To work around this problem, a DC voltage source was connected to the gate of M7 to hold the gate at a constant voltage level of $847 \mathrm{mV}$ when the switch was in the OFF state. The value of DC voltage source was determined by running a transient simulation and observing the value of the voltage obtained when the switch went from the closed to open state, and, while in the open state, the voltage value that still produced the desired output frequency (Figure 3.9 in subsection 3.3.1 illustrates that this bias value results in the correct VCO output frequency). This test could also have been done by using an initial condition on the capacitor.

The phase noise simulation results obtained from Cadence were already presented in subsection 3.4.3.

To verify the test methodology used, and therefore to verify the result, it was decided to implement the schematic using the Agilent Advanced Design System (ADS) simulator. A discussion of the simulation using ADS is described in the next section. 


\subsection{ADS Simulations}

In ADS it is possible to generate a final solution file from one simulation and read it in as the initial starting point for the next simulation. ADS uses a harmonic balance simulation to produce phase noise measurements. As before, in SpectreRF, the main switch, M9, was set to the ON state, and the harmonic balance was simulated, and a final solution file was generated. This file was then used as the initial starting point for the harmonic balance simulation in which the main switch, M9, was set to the OFF state. The testbench for the simulation is shown in Figure 4.9, including the device sizes that were used for each of the transistors. As can be seen in the testbench, the dummy switch, M10, was not included during the simulations used to measure the phase noise of the VCO as it further complicated the simulation by requiring an additional clock, and it has already been shown (refer to subsection 3.4.3, Figure 3.15) that the dummy switch, M10, contributed only a very small amount (on the order of $8.4 \mathrm{e}-19$ ) of noise to the circuit, and therefore does not impact the phase noise value.

The inductor is a $6.1 \mathrm{nH}$ rectangular spiral inductor and was modelled in the simulation using the model presented in [8] as is shown in Figure 4.2. The inductor model was developed from the RF models provided by CMC. Details of the VCO structure, including the inductor model, were provided in section 4.1. Other elements of interest in the testbench shown in Figure 4.9 include the use of a piece-wise linear component, VPWL, which provided the pulse to kick-start the VCO on startup, and the "OscPort2" component required for oscillator analysis in ADS [44]. This probe component was inserted differentially into the oscillator loop following the instructions provided in ADS' help files. The control voltage, Vctrl, for the VCO, which is connected to the MOSCAPs used for tunning, was set to $1.5 \mathrm{~V}$. The supply voltage, VDD, was set to $1.8 \mathrm{~V}$, and the main switch, M9, had a supply voltage to its gate of $1.8 \mathrm{~V}$ when the switch was turned on, and $0 \mathrm{~V}$ when the switch was turned off. The offset frequencies between $90 \mathrm{kHz}$ and $1.2 \mathrm{MHz}$ were swept. These values were chosen as the focus of the research was on the noise at close-in frequencies. The main frequencies that were considered during this research 
were $100 \mathrm{kHz}, 200 \mathrm{kHz}, 600 \mathrm{kHz}$ and $1 \mathrm{MHz}$. These values were chosen not only because they are close-in frequencies, but also because they are frequency offset values that were often quoted for research papers involving VCO (refer to Table 2.1 and Table 2.2). The same offset frequencies were measured in both Cadence and ADS to allow for a direct comparison of values, as is shown in Figure 4.10.

The TSMC $0.18 \mu \mathrm{m}$ kit available from CMC was used for the MOSFET mixed models. The parameters used to setup the harmonic balance test are shown in Table 4.4 (any other parameters not listed were kept as the default values):

Table 4.4: Harmonic Balance Simulation Para
\begin{tabular}{|c||c|}
\hline Frequency & $1.4 \mathrm{GHz}$ \\
\hline Order & 7 \\
\hline Noise: Sweep Type & Log \\
\hline Noise: Start & $90 \mathrm{kHz}$ \\
\hline Noise: Stop & $1.2 \mathrm{MHz}$ \\
\hline Noise: Pts./decade & 100 \\
\hline Noise outputs & Vout+, Vout- \\
\hline Oscillator & use Oscport \\
\hline
\end{tabular}

The results, as are shown in Figure 4.11, clearly illustrate a decrease in the phase noise between the case when the switch is turned on and the case when the switch is turned off. The decrease in phase noise on average (taking the decrease in phase noise by measuring the difference in phase noise between the case when the switch is on and when the switch is off, and averaging the decreases values over the offset frequencies measured) is approximately $6 \mathrm{~dB}$, as can be seen in Figure 4.12. There is an slight increasing trend that is observable in this plot, indicating that there is a larger difference between the phase noise when the switch is in the on and off state as the offset frequency increases. However, the curve is steeper for the lower frequencies, with a slope of 0.2 between $100 \mathrm{kHz}$ and $500 \mathrm{kHz}$, then at the higher frequencies, which has a slope of 0.08 between $500 \mathrm{kHz}$ and $1 \mathrm{MHz}$. This indicates that the slope is starting to level off as thermal noise becomes the dominate noise source, therefore showing that the switched-bias circuit is still better suited for close-in frequencies.

These results closely match the results obtained from SpectreRF, with a maximum 

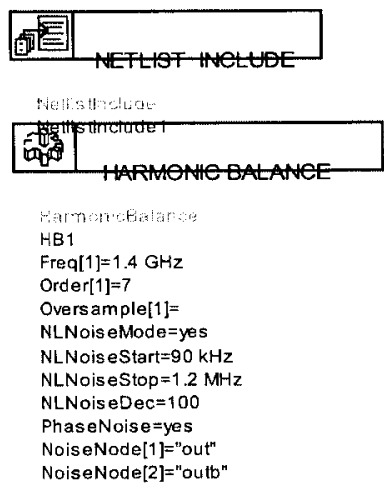

NoiseNode[2]="outb"

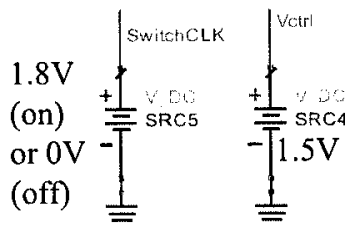

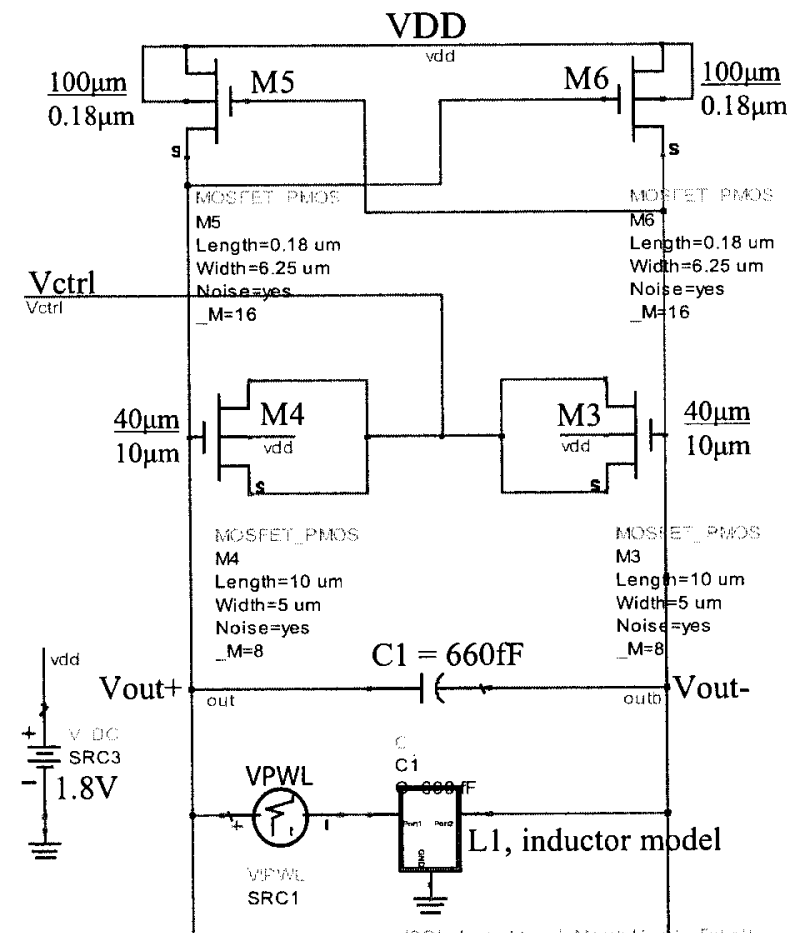

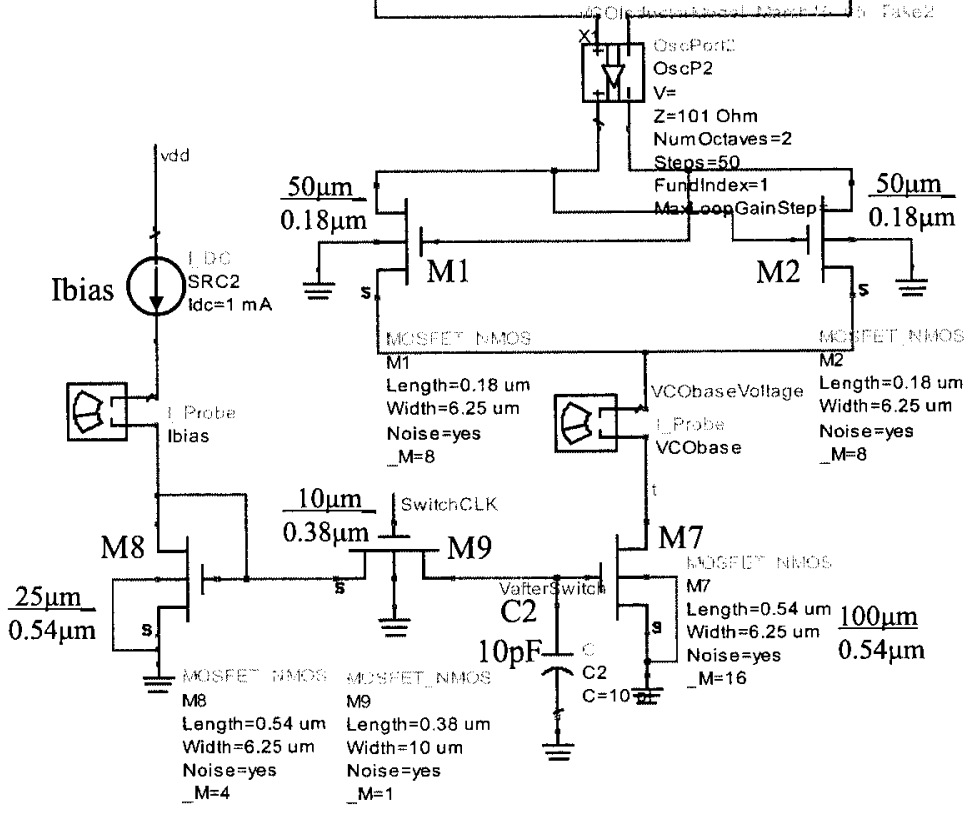

Figure 4.9: Testbench for the ADS phase noise simulation. 

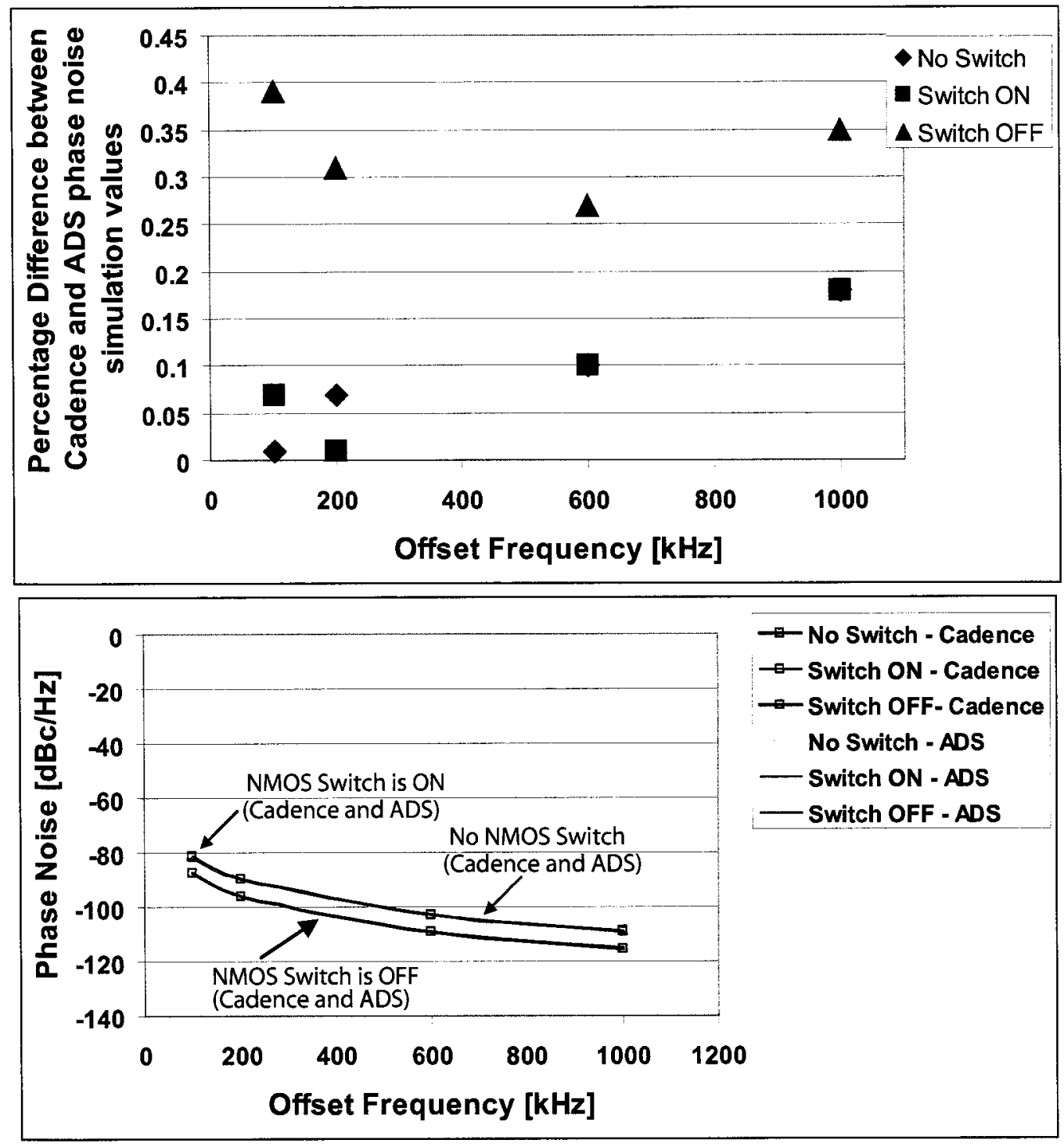

Figure 4.10: Single-sideband (SSB) phase noise simulations over various offset frequencies. The phase noise values for each of the three states of the circuit (no switch, with the switch on and with the switch off) were plotted for both the Cadence SpectreRF and ADS simulations. The top plot also shows the percentage difference in the phase noise values between the Cadence and ADS simulations. 


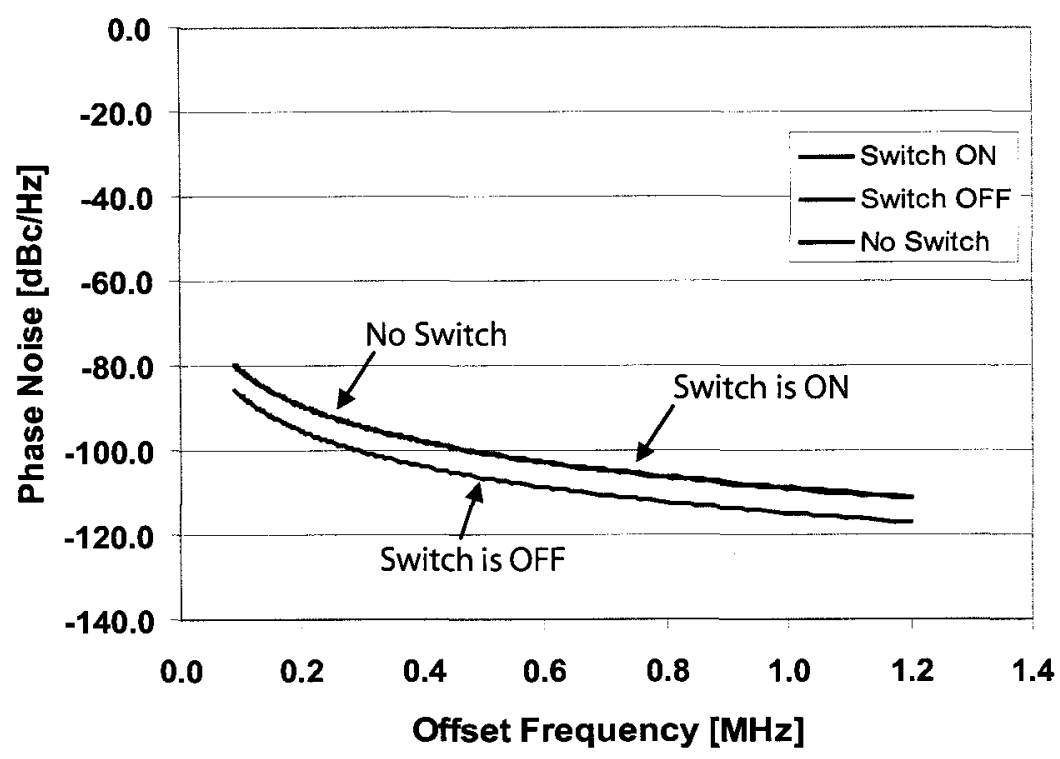

Figure 4.11: Phase noise simulations over various offset frequencies. The phase noise values for the circuit when the switch is ON and OFF are plotted. This simulation shows the decrease in phase noise when the switch is turned off. In addition, it can be seen from this graph that the addition of the switched-bias circuitry did not result in a noticeable change in phase noise, as the case for the phase noise with no switch is practically identical to the case for the phase noise when the switch is turned on. 


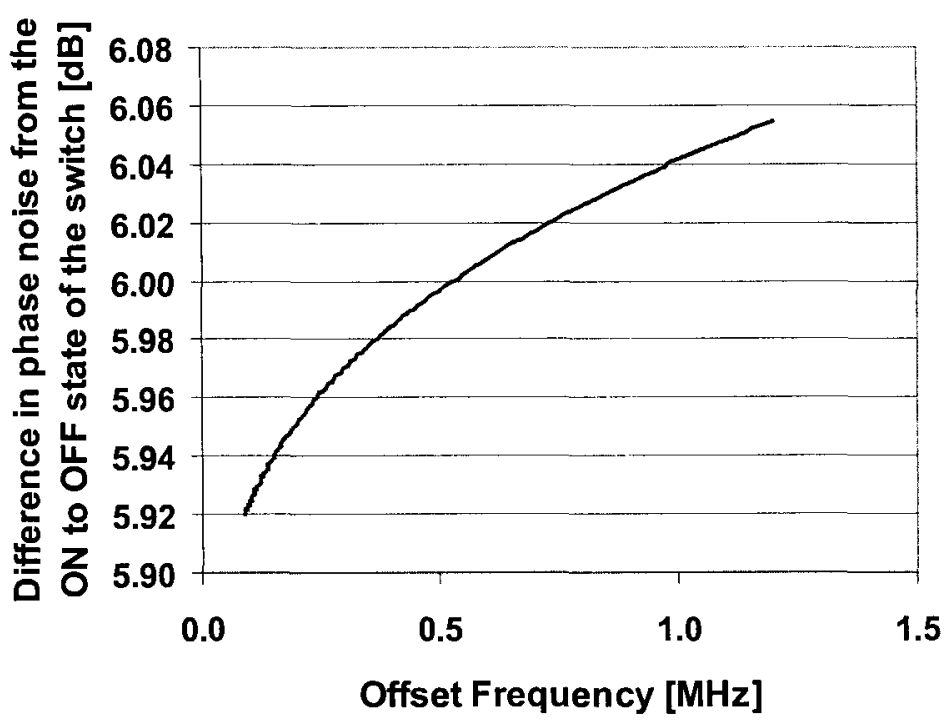

Figure 4.12: Decrease in phase noise as the switch goes from the ON state to the OFF state. The value of the decrease in phase noise varies by $2.2 \%$ over the simulated offset frequencies.

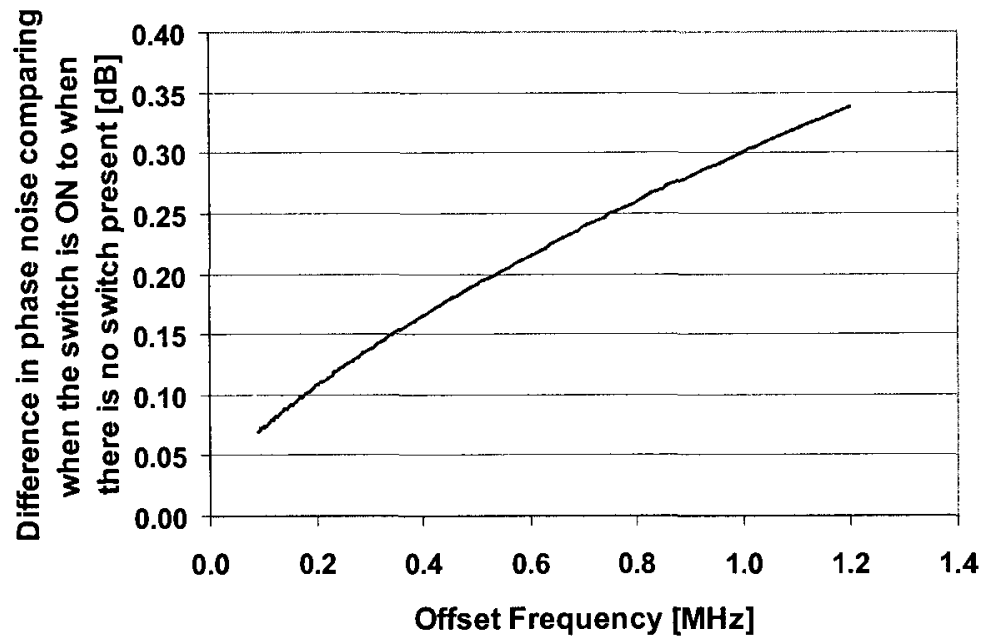

Figure 4.13: Plot of the difference in phase noise for the VCO when the switch is ON and when there is no switch present in the design. The difference was calculated to be, on average, $0.3 \mathrm{~dB}$ over the range of simulated offset frequencies. 
discrepancy of $0.39 \%$ as is shown in Figure 4.10. Figure 4.10 plots the phase noise values from both the Cadence and ADS simulation, as well as showing the percentage difference between the phase noise values from the two simulators, for cases of the main switch, M9, a) not being included in the circuit, b) switch being on and c) switch being off. These results indicate that the method used to measure the change in VCO phase noise in SpectreRF is valid. In addition, Figure 4.11 and Figure 4.13 show that the extra switched-bias circuitry does not contribute a noticeable amount of additional noise. The VCO phase noise was measured both with and without the switch in the VCO and as can be seen from Figure 4.11 the simulation without the switch, and the simulation with the switch in the ON state are almost identical, with only an average difference of $0.3 \mathrm{~dB}$ (this average was measured in the same manner as the average decrease in phase noise was measured, though in this situation the difference between the case when the switch was on and when the switch was left out of the VCO bias circuit was considered). To further emphasis the fact that the addition of the switched-bias circuit did not add a noticeable amount of noise, the difference in noise between the case when there as no switch in the bias network, and when the switch was $\mathrm{ON}$ is plotted in Figure 4.13. There is an increasing trend that is observable in this plot. This increase indicates that the flicker noise of the NMOS switch which is dominate in the lower offset frequencies has a lower impact on the overall phase noise then the thermal noise of the NMOS switch which dominates at the higher offset frequencies. This suggests that the switched-bias technique would be less beneficial at higher offset frequencies as the added switched-bias circuit begins contributing more noise to the circuit itself, re-enforcing the idea presented in subsection 2.3.1 that the focus for this technique is for close-in frequencies.

\subsection{Impact of spurs on radio system}

Spurs are undesired signals in a radio system that can interfere with the desired signal. They can result from various different sources, including due to the various components within a radio system. For example, mismatch of either the current or sizes of the charge pump can lead to reference spurs, which will be discussed in more detail within this 
section.
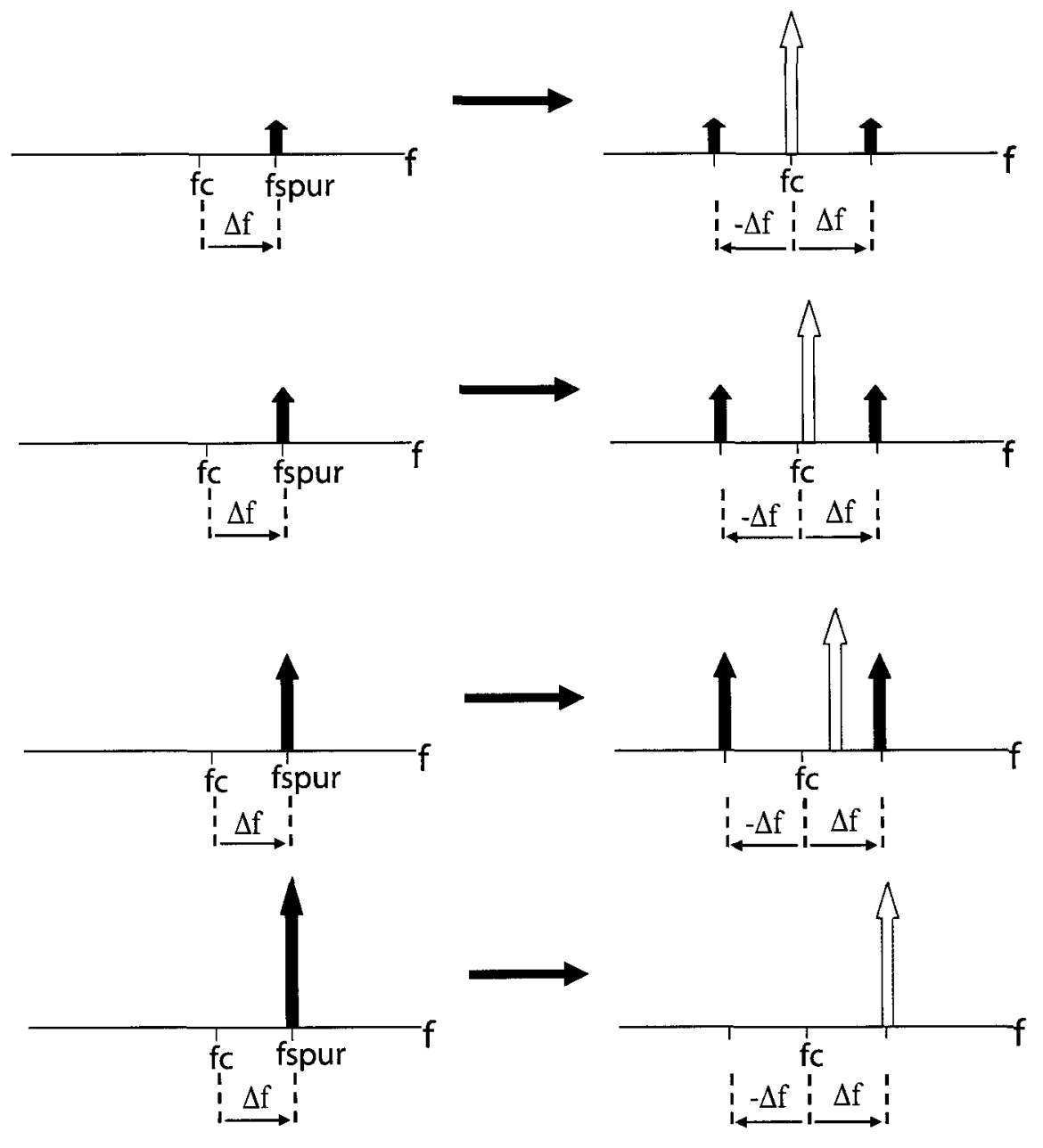

Figure 4.14: The plot shows the impact of spurs on VCO frequency. As the amplitude of the spurious signal increases, the frequency begins shifting away from the desired carrier frequency and towards the spurious frequency.

Spurs close in frequency to the VCO resonant frequency can cause the VCO frequency to shift when the spurs amplitude becomes high enough. This effect is illustrated in Figure 4.14. It is discussed in [45], "random and discrete frequency components cause a broad skirt and spurious peaks" in the VCO frequency spectrum. It is stated in [45] that "the discrete spurious components could be caused by known clock frequencies in the signal source, power line interference, and mixer products. The broadening caused by random noise fluctuations is due to phase noise. It can be the result of thermal noise, shot noise 
and/or flicker noise in active and passive devices." The impact of spurious signals can be seen when the VCO is placed within a system, for example in a phase-locked loop (PLL), in which the VCO provides the local oscillator (LO) frequency. It is necessary that the VCO frequency be as stable as possible to provide the correct frequency to the rest of the PLL system. Therefore spurs must be minimized to avoid these undesired signals mixing with the VCO frequency and causing a shift in the VCO frequency.

The effect of reference spurs are well known in PLLs. It is discussed in [45] that in "an integer-N PLL, reference spurs are caused by the fact that the charge pump output is being continuously updated at the reference frequency rate." As there is continually current pulses being outputted from the charge pump, the DC voltage that drives the $\mathrm{VCO}$ is modulated by a signal of the reference frequency for the phase frequency detector in the system. This modulation produces reference spurs in the output of the VCO. These reference spurs occur at offset frequencies that are integer multiples of the reference frequency. The reference spurs due to the charge pump lead to reference feedthrough, and result in the toggling of the charge pump output even under a locked condition.

The impact of the spurs that could potentially be generated by the switch in the switched-bias circuit will be investigated in the next section.

\subsection{Analysis of Switching Noise}

The noise associated with the switched-bias circuit must be considered. This noise consists of two parts: the flicker noise, and the sampling noise (i.e. the $\mathrm{kT} / \mathrm{C}$ noise). The impact the switching noise will have on the overall phase noise of the VCO must be investigated and the value of the flicker noise that is being transferred to the bias due to the switching of the clock must also be evaluated.

The flicker noise will be considered first. Due to the toggling of the switch at the sampling frequency using a 50\% duty cycle square wave clock input, the flicker noise will appear in the output spectrum at odd harmonics of the sampling frequency, as can be seen in Figure 4.15.

To begin determining the impact of the flicker noise on the gate voltage of the tail 


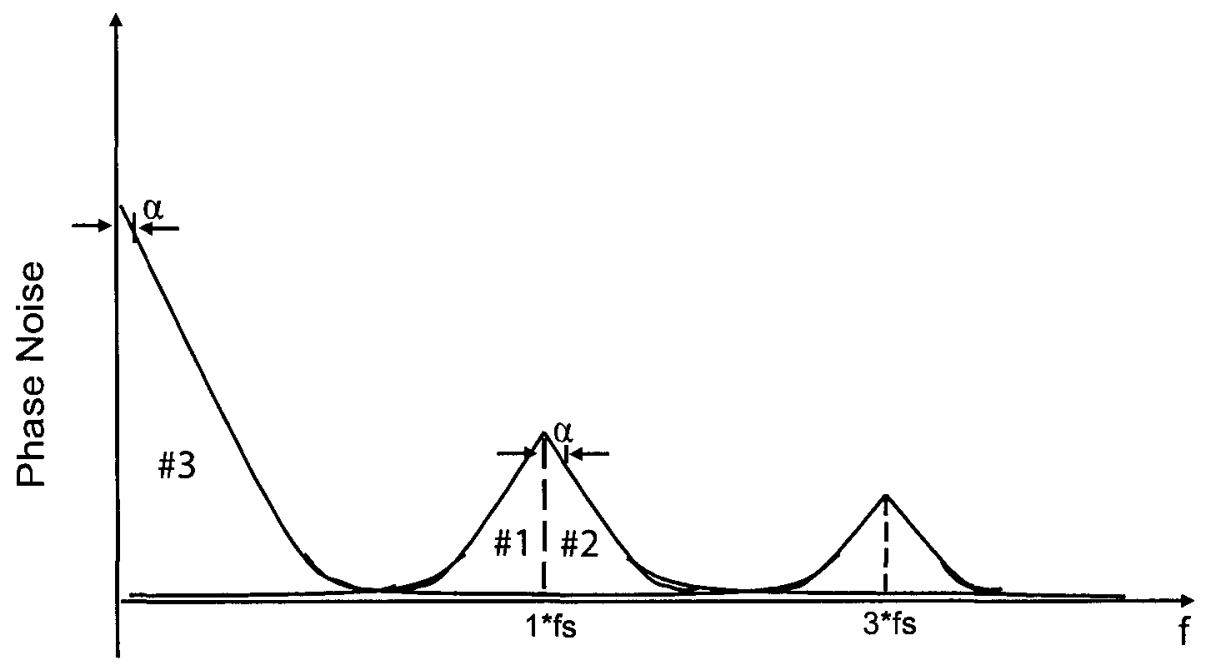

Figure 4.15: This figure illustrates how the flicker noise appears at the odd harmonics of the sampling frequency. The plot has been divided into three sections, labelled as \#1, \#2 and $\# 3$, to determine the total flicker noise from the switched-bias circuit. The variable $\alpha$ denotes the minimum frequency for which the flicker noise can be determined. 
current transistor, M7, the overall flicker noise can be divided into the three regions shown in Figure 4.15. This diagram provides an illustration as to how the overall flicker noise can be determined. Region \#3 is the expected 1 /f region associated with flicker noise, which was discussed in subsections 2.3.2 and 2.6.1. Regions \#1 and \#2 show the flicker noise from region \#3 which is replicated at multiples of $(2 \mathrm{k}-1)$ of the sampling frequency, $\mathrm{f}_{s}$ (i.e. the clock frequency) where $\mathrm{k}$ is series index (as can be seen in (4.7.1)), which goes from zero to infinity. This noise is a result of the switching nature of the switched-bias circuit, wherein the switch is toggled using a square wave clock input. As is seen in Figure 4.15, the amplitude of the transferred flicker noise decreases as the overall frequency increases, which is due to the coefficients associated with the Fourier series of the clock square waveform. The equations used to determine the noise in regions \#1, \#2 and \#3 are given as,

Region \#1:

$$
\text { FNoise } e_{\text {region } 1}=\sum_{k=0}^{\infty} \int_{0}^{((2 k+1)-\alpha)}\left(\frac{4}{\pi} \frac{1}{(2 k+1)}\right)^{2} \frac{1}{1+\left(2 \pi f R_{o n} C\right)^{2}} \frac{k_{f}}{2 W L C_{o x}\left((2 k+1) f_{s}-f\right)} d f
$$

where $\mathrm{k}$ is the series index, $\mathrm{R}_{\text {on }}$ is the on resistance of the switch transistor, M9, C is the bias retention capacitor $(\mathrm{C} 2), \mathrm{k}_{f}$ is the flicker noise process-dependent constant, $\mathrm{C}_{o x}$ is the gate capacitance per unit area, $\mathrm{W}$ and $\mathrm{L}$ are, respectively, the width and length of the switch transistor, $\mathrm{f}_{s}$ is the sampling (i.e. clock) frequency, $\alpha$ is the minimum frequency for which the original flicker noise can be determined, and $f$ is the frequency. From the CMOSP18 model file, $\mathrm{k}_{f}$ is $4.0 \times 10^{-25} \mathrm{~V}^{2} \mathrm{~F}$, and $\mathrm{C}_{o x}$ is $8.46 \times 10^{-3} \mathrm{Fm}^{-2}$. From simulations, $R_{\text {on }}$ was determined to be $347.2 \Omega$, however this value is an estimation as the resistance value will vary as the clock switches. The sampling frequency was set to $1 \mathrm{MHz}$, and from Figure 4.9 it is seen the $\mathrm{C}$ is $10 \mathrm{pF}, \mathrm{W}$ is $10 \mu \mathrm{m}$ and $\mathrm{L}$ is $0.38 \mu \mathrm{m}$.

The square wave coefficient is given by the first two terms in (4.7.1), the third term results from the filter nature of the switched bias circuit (i.e. the flicker noise will be filtered by the $\mathrm{RC}$ time constant that results due to the bias retention capacitor, $\mathrm{C} 2$, and the on resistance of the switch transistor, M9). The last term is the expression for flicker 
noise in a MOSFET. The expression is summed as a series as the square wave results in a Fourier series that continues to infinity.

The equation for region \#2 is very similar to the equation for region $\# 1$, however, the limits are changed to take into account the second half of the transferred flicker noise,

$$
\text { FNoise } \text { region } 2=\sum_{k=0}^{\infty} \int_{((2 k+1)+\alpha)}^{f_{\text {high }}}\left(\frac{4}{\pi} \frac{1}{(2 k+1)}\right)^{2} \frac{1}{1+\left(2 \pi f R_{o n} C\right)^{2}} \frac{k_{f}}{2 W L C_{o x}\left(f-(2 k+1) f_{s}\right)} d f
$$

The last section of noise to consider is the original flicker noise, which is the noise component that is being transferred by the sampling frequency of the clock square wave. This noise component is given by region $\# 3$ in Figure 4.15 , and is defined as,

$$
F N o i s e_{\text {region } 3}=\int_{\alpha}^{f_{\text {high }}} \frac{1}{1+\left(2 \pi f R_{o n} C\right)^{2}} \frac{k_{f}}{2 W L C_{o x f}} d f
$$

The variable $\alpha$ defines the lower frequency limit for flicker noise, as the integral of the original flicker noise expression results in the $\ln$ function. As the $\ln$ of zero (i.e. $\ln (0)$ ) is not defined and would yield an infinite value for the total noise, it is required to define a lower frequency limit, $\mathrm{f}_{\text {low }}$, which is equal to $\alpha$ as is shown in Figure 4.15. This conclusion is supported by [46, pp.217] which states that extending the lower limit to zero simply means that one is interested in arbitrarily slow noise components, and in reality over a very long length of time at very slow rates these noise components become "indistinguishable from thermal drift or the aging of devices." It is also necessary to set an upper bound on the frequency for this region, as the ln of infinity equals infinity, and the equation would go to zero as it would contain both a positive and negative infinity term. This upper limit applies to both the original flicker noise and the flicker noise associated with region \#2. The upper frequency term, $\mathrm{f}_{\text {high }}$, can be set to the cut-off frequency of the $\mathrm{RC}$ filter that is formed due to the resistance of the switch transistor, $\mathrm{R}_{o n}$, and the bias retention capacitor,C. Above this point, the filter will attenuate the flicker noise in this region. Therefore $\mathrm{f}_{\text {high }}$ is calculated using,

$$
f_{\text {high }}=\frac{1}{2 \pi R_{o n} C}
$$


The first step in determining the noise current at the bias is to determine the noise at the gate of the tail current transistor, M7. This noise is a combination of the flicker noise from regions \#1, \#2 and \#3, and the $\mathrm{kT} / \mathrm{C}$ noise that is associated with sample and hold circuits. The analysis and derivation for the $\mathrm{kT} / \mathrm{C}$ noise has been done by Kundert and is presented in [47]. To determine the contribution of the flicker noise, the equations shown in (4.7.1) to (4.7.3) must be evaluated. The Mathematica software program was used to evaluate the integrals, and the results are as follows:

For region \#1:

$$
\left.\begin{array}{l}
\int_{0}^{((2 k+1)-\alpha)}\left(\frac{4}{\pi} \frac{1}{(2 k+1)}\right)^{2} \frac{1}{1+\left(2 \pi f R_{o n} C\right)^{2}} \frac{k_{f}}{2 W L C_{o x}\left((2 k+1) f_{s}-f\right)} d f \\
{\left[\begin{array}{l}
-4 C f_{s}(2 k+1) \pi R_{o n} \cdot \\
\arctan \left(2 C \pi R_{o n}\left(\begin{array}{l}
(2 k+1) f_{s} \\
-\alpha
\end{array}\right)\right)
\end{array}\right]} \\
-\ln \left(\begin{array}{l}
1+ \\
4 C^{2} \pi^{2} R_{o n}^{2}\left(\begin{array}{l}
(2 k+1) f_{s} \\
-\alpha
\end{array}\right)
\end{array}\right)
\end{array}\right]
$$

For region \#2:

$$
\begin{aligned}
& \int_{((2 k+1)+\alpha)}^{\left(f_{\text {high }}\right)}\left(\frac{4}{\pi} \frac{1}{(2 k+1)}\right)^{2} \frac{1}{1+\left(2 \pi f R_{o n} C\right)^{2}} \frac{k_{f}}{2 W L C_{o x}\left(f-(2 k+1) f_{s}\right)} d f \\
& =\left[\frac{8 k_{f}}{C_{o x} W L \pi^{2}(2 k+1)^{2}\left(2+8 C^{2} f_{s}^{2}(1+2 k)^{2} \pi^{2} R_{o n}^{2}\right)}\right]\left[\begin{array}{l}
4 C f_{s}(2 k+1) \pi R_{o n} . \\
{\left[\begin{array}{l}
\arctan \left(2 C \pi R_{o n}\left(\begin{array}{l}
(2 k+1) f s \\
+\alpha
\end{array}\right)\right) \\
-\frac{\pi}{4}
\end{array}\right]} \\
+\ln \left(\begin{array}{l}
1+ \\
4 C^{2} \pi^{2} R_{o n}^{2}\left(\begin{array}{l}
(2 k+1) f s \\
+\alpha
\end{array}\right)
\end{array}\right) \\
+\ln (2)
\end{array}\right]
\end{aligned}
$$

Lastly, setting $\alpha$ to the lower frequency limit, $\mathrm{f}_{\text {low }}$, the integral for region $\# 3$ is, 


$$
\begin{aligned}
& \int_{f_{\text {low }}}^{f_{\text {high }}} \frac{1}{1+\left(2 \pi f R_{o n} C\right)^{2}} \frac{k_{f}}{W L C_{\text {ox }} f} d f \\
& =\frac{k_{f}}{2 W L C_{o x}}\left(\begin{array}{l}
-2 \ln \left(f_{\text {low }}\right)+\ln \left(1+4 C^{2} \pi^{2} R_{\text {on }}^{2} f_{\text {low }}^{2}\right)+ \\
2 \ln \left(f_{\text {high }}\right)-\ln \left(1+4 C^{2} \pi^{2} R_{\text {on }}^{2} f_{\text {high }}^{2}\right)
\end{array}\right) \\
& =\frac{k_{f}}{2 W L C_{o x}}\left(\begin{array}{l}
-2 \ln \left(f_{\text {low }}\right)+\ln \left(1+4 C^{2} \pi^{2} R_{o n}^{2} f_{\text {low }}^{2}\right)+ \\
2 \ln \left(\frac{1}{2 \pi R_{o n} C}\right)-\ln \left(1+4 C^{2} \pi^{2} R_{o n}^{2}\left(\frac{1}{2 \pi R_{o n} C}\right)^{2}\right)
\end{array}\right)
\end{aligned}
$$

The flicker noise integrals derived for regions \#1 and \#2 must be summed as the noise in these regions result from the sampling effect on the flicker noise due to the toggling of the switch by a square wave clock signal. As a result, for regions \#1 and \#2, the final voltage noise components are:

Region \#1:

$$
\sum_{k=0}^{\infty} \frac{-8 k_{f}}{C_{o x} W L \pi^{2}(2 k+1)^{2}\left(2+8 C^{2} f_{s}^{2}(1+2 k)^{2} \pi^{2} R_{o n}^{2}\right)}\left[\begin{array}{l}
-4 C f_{s}(2 k+1) \pi R_{o n} \\
\arctan \left(2 C \pi R_{o n}\left(\begin{array}{l}
(2 k+1) f_{s} \\
-\alpha
\end{array}\right)\right) \\
-\ln \left(\begin{array}{l}
1+ \\
4 C^{2} \pi^{2} R_{o n}^{2}\left(\begin{array}{l}
(2 k+1) f_{s} \\
-\alpha
\end{array}\right)
\end{array}\right)
\end{array}\right]
$$

Region \#2:

$$
\sum_{k=0}^{\infty}\left[\frac{8 k_{f}}{C_{o x} W L \pi^{2}(2 k+1)^{2}\left(2+8 C^{2} f_{s}^{2}(1+2 k)^{2} \pi^{2} R_{o n}^{2}\right)}\right]\left[\begin{array}{l}
4 C f_{s}(2 k+1) \pi R_{o n} . \\
{\left[\begin{array}{l}
\arctan \left(2 C \pi R_{o n}\left(\begin{array}{l}
(2 k+1) f s \\
+\alpha
\end{array}\right)\right) \\
-\frac{\pi}{4}
\end{array}\right]} \\
+\ln \left(\begin{array}{l}
1+ \\
4 C^{2} \pi^{2} R_{o n}^{2}\left(\begin{array}{l}
(2 k+1) f s \\
+\alpha
\end{array}\right)
\end{array}\right) \\
+\ln (2)
\end{array}\right]
$$

Therefore the total noise voltage seen at the gate of the bias transistor (i.e. M7, refer to Figure 4.9 for the schematic of the circuit) is the sum of the flicker noise in regions 


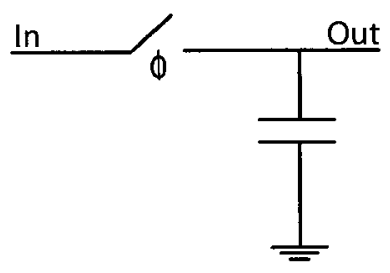

Figure 4.16: Simple track and hold circuit, which is equivalent to the switched-bias circuitry.

$\# 1, \# 2$ and \#3, as well the $\mathrm{kT} / \mathrm{C}$ noise that is generally associated with a track and hold circuit, which is equivalent to the switched-bias circuit, as is seen in the simple track and hold schematic shown in Figure 4.16. The full analysis and derivation of the $\mathrm{kT} / \mathrm{C}$ noise for a track and hold circuit is given by Kundert in [47]. Therefore the final expression for the noise voltage at the gate of M7 is,

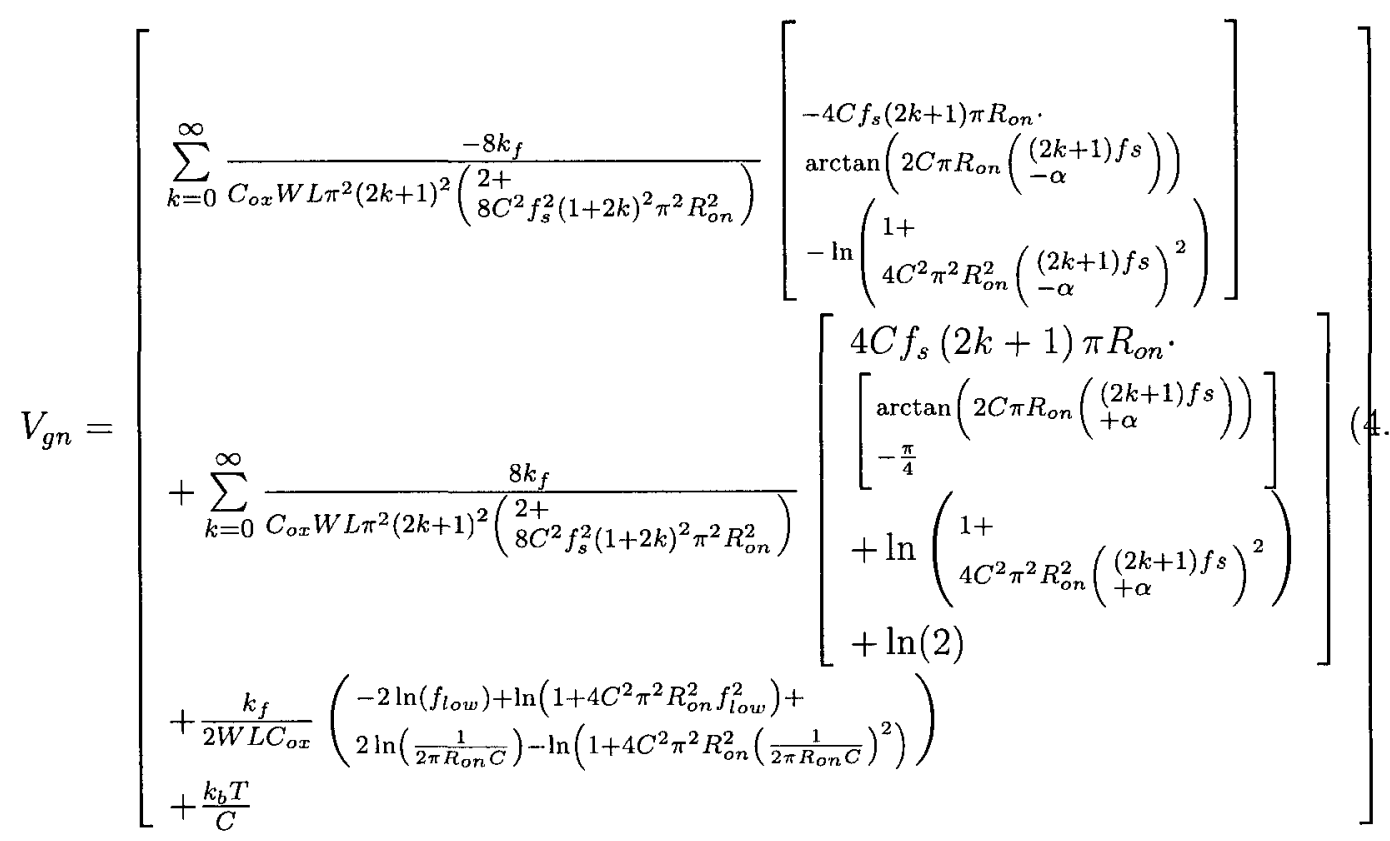

where $\mathrm{C}_{o x}$ is the gate capacitance per unit area, $\mathrm{W}$ and $\mathrm{L}$ are, respectively, the width and length of the switch transistor, $\mathrm{R}_{o n}$ is the on resistance of the switch transistor, $\mathrm{C}$ is the bias retention capacitor, $\mathrm{k}_{f}$ is the flicker noise process-dependent constant, $\mathrm{k}$ is the series index for the summations in region $\# 1$ and region $\# 2, \mathrm{k}_{b}$ is Boltzmann's constant, $\mathrm{T}$ is 
the temperature in Kelvins, $\mathrm{f}_{s}$ is the sampling (i.e. clock) frequency, and $\mathrm{f}_{\text {low }}$ is the lower limit frequency for the flicker noise in region \#3.

In $[2,48]$ Hajimiri and Lee introduce a new method to calculate the phase noise in a VCO. In particular, in [2], the impact of noise in the bias tail current of an LC oscillator on the phase noise is investigated. It is shown that the noise in the bias tail current does result in a change in the phase noise of the VCO. As a result, to see the impact of the noise at the gate of the tail current bias transistor, M7, due to the noise from the switched-bias circuit, on the overall phase noise, the gate voltage value must be converted to the bias current. This is done by multiplying by the square of the transconductance of the tail current bias transistor, (i.e. M7) which is in the triode region of operation,

$$
I_{b n}=\left(V_{g n}\right)\left(g_{m}^{2}\right)
$$

Substituting in the expression for $\mathrm{V}_{g n}$ from (4.7.10), the complete expression for the bias noise is,

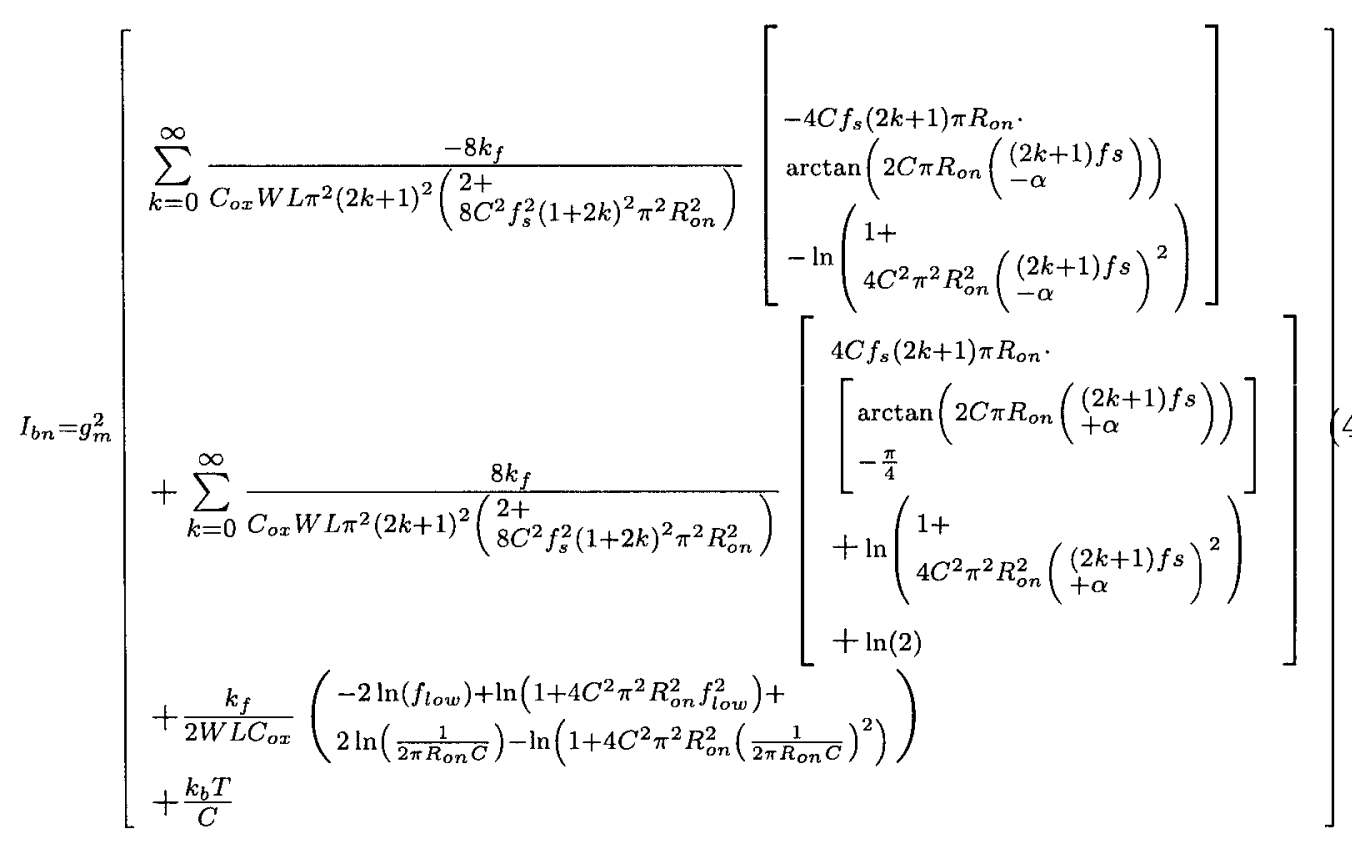

It should be noted that, as can be seen from Figure 4.15, the flicker noise from region \#1 overlaps with the flicker noise from regions \#2 and \#3 and this overlap increases as 
the sampling frequency, $\mathrm{f}_{s}$, decreases. Therefore the flicker noise will be higher for lower sampling frequencies.

\subsubsection{Transient Noise Simulation}

As mentioned in the previous section, it has been shown by Hajimiri and Lee in [2] that there is a link between the bias current noise and phase noise. Therefore to investigate the noise in the bias current, this noise was simulated through transient simulations, using the testbench shown in Figure 4.17. A transient simulation was run for $1 \mathrm{~ms}$ both with and without noise, and a DFT was performed on the bias current signal to obtain the frequency spectrum, as shown in Figure 4.18 and Figure 4.19. The transient with noise simulation used minimum and maximum noise frequencies of $100 \mathrm{kHz}$ and $5 \mathrm{GHz}$, respectively.

As expected, the bias current has a frequency that is double the VCO oscillation frequency. As explained in [2], the reason for the increase in frequency is that the tail node is "pulled up every time one of the differential NMOS transistors turns on," and as a result, the tail node moves at twice the frequency of the differential voltage.

Figure 4.18 shows a comparison of the bias current spectrum for the case of the transient simulation both with and without noise. It is seen that the addition of the noise leads to a shift in the center frequency, as well as some spreading of the signal. Figure 4.19 provides a zoomed in view of the spectrum, and spurs due to the clock frequency can be observed at offsets from the center frequency. These spurs will be discussed further in Section 4.8 .

It is also of interest to plot the VCO output and do a comparison between the case when the switch in the switched-bias circuit is switching, and the case when there is no switched-bias circuit included in the VCO. A transient simulation with noise, and a clock frequency of $1 \mathrm{MHz}$, was run for $1 \mathrm{~ms}$ using the testbench shown in Figure 4.17. The transient with noise simulation used minimum and maximum noise frequencies of $100 \mathrm{kHz}$ and $5 \mathrm{GHz}$, respectively. For the second case, for the VCO without the switch, the testbench Figure 4.20 was used, and again the transient simulation was run for $1 \mathrm{~ms}$. 

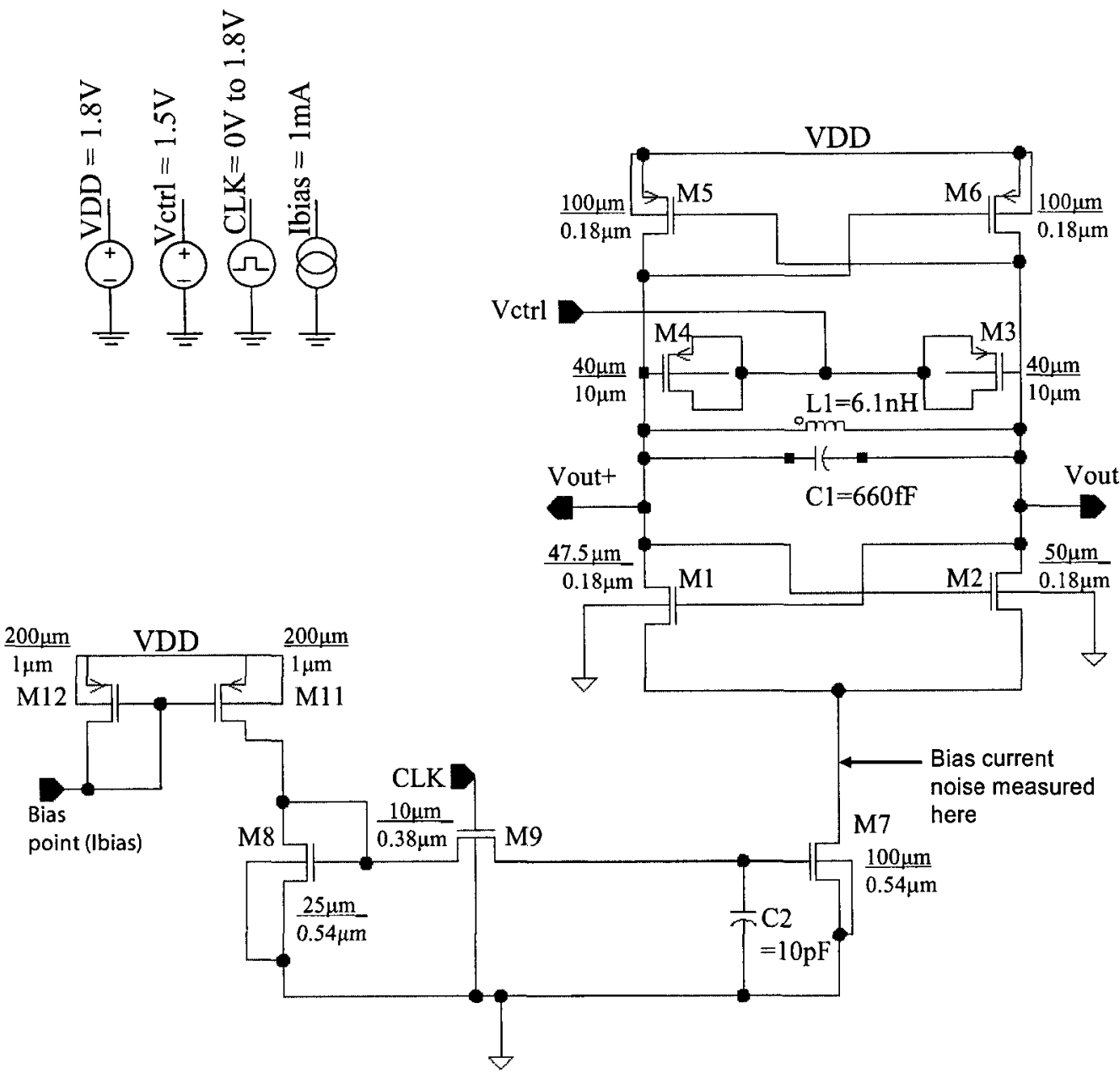

Figure 4.17: Testbench used to measure the bias noise and the VCO output. The transient simulation was run both with and without noise, for $1 \mathrm{~ms}$. The bias current was measured where it is indicated in the testbench. The current waveform and the VCO output were then transformed into their equivalent frequency spectrum using a DFT with a resolution of $10 \mathrm{kHz}$, using 524,288 sample points. The clock was toggled at a rate of $1 \mathrm{MHz}$. 


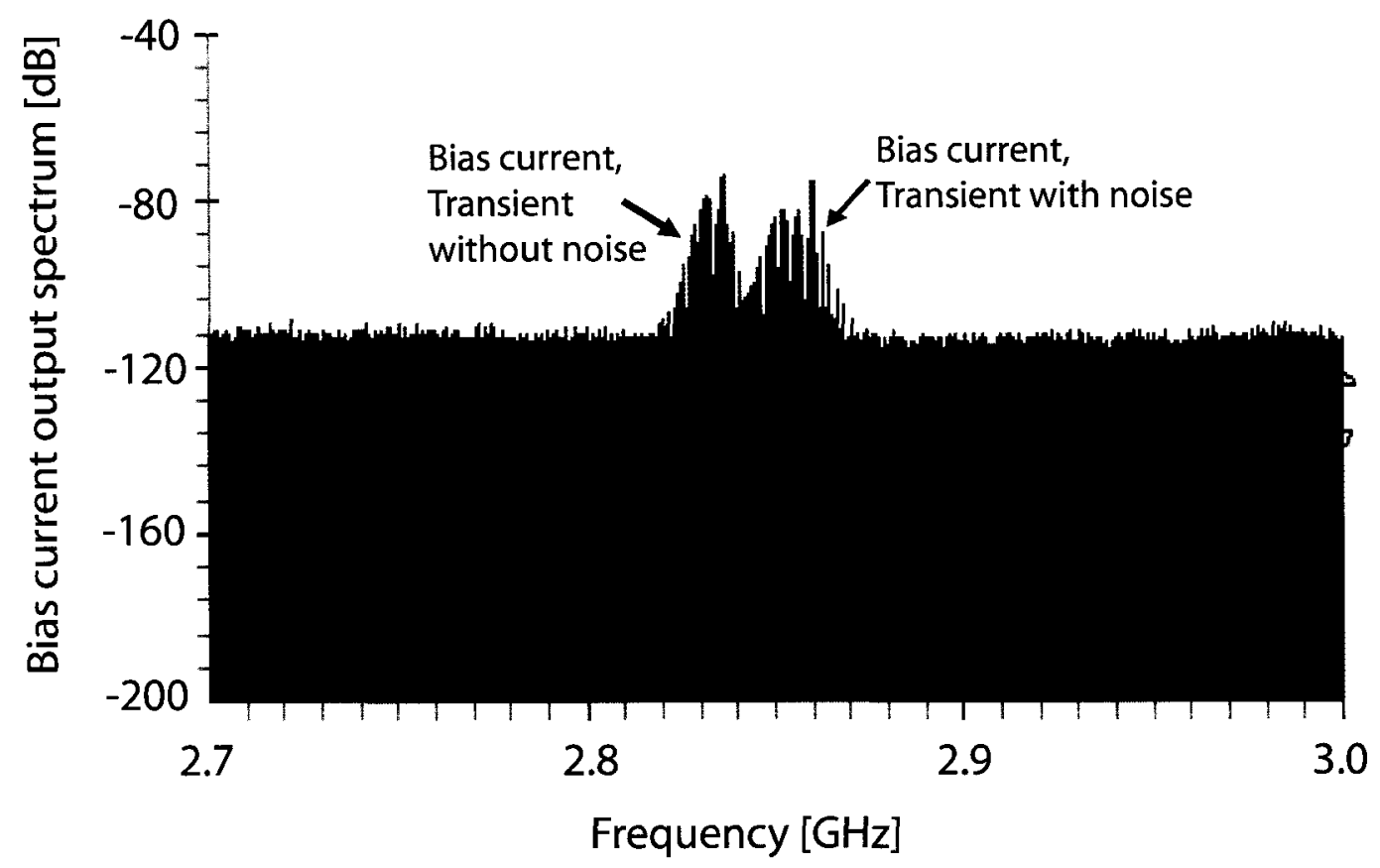

Figure 4.18: Spectrum of the bias current for the case of the transient with and without noise. The current waveform was transformed using a DFT with a bin size of $10 \mathrm{kHz}$, using 524,288 sample points. The clock was toggled at a rate of $1 \mathrm{MHz}$. It is seen that a frequency shift occurred due to the noise. 


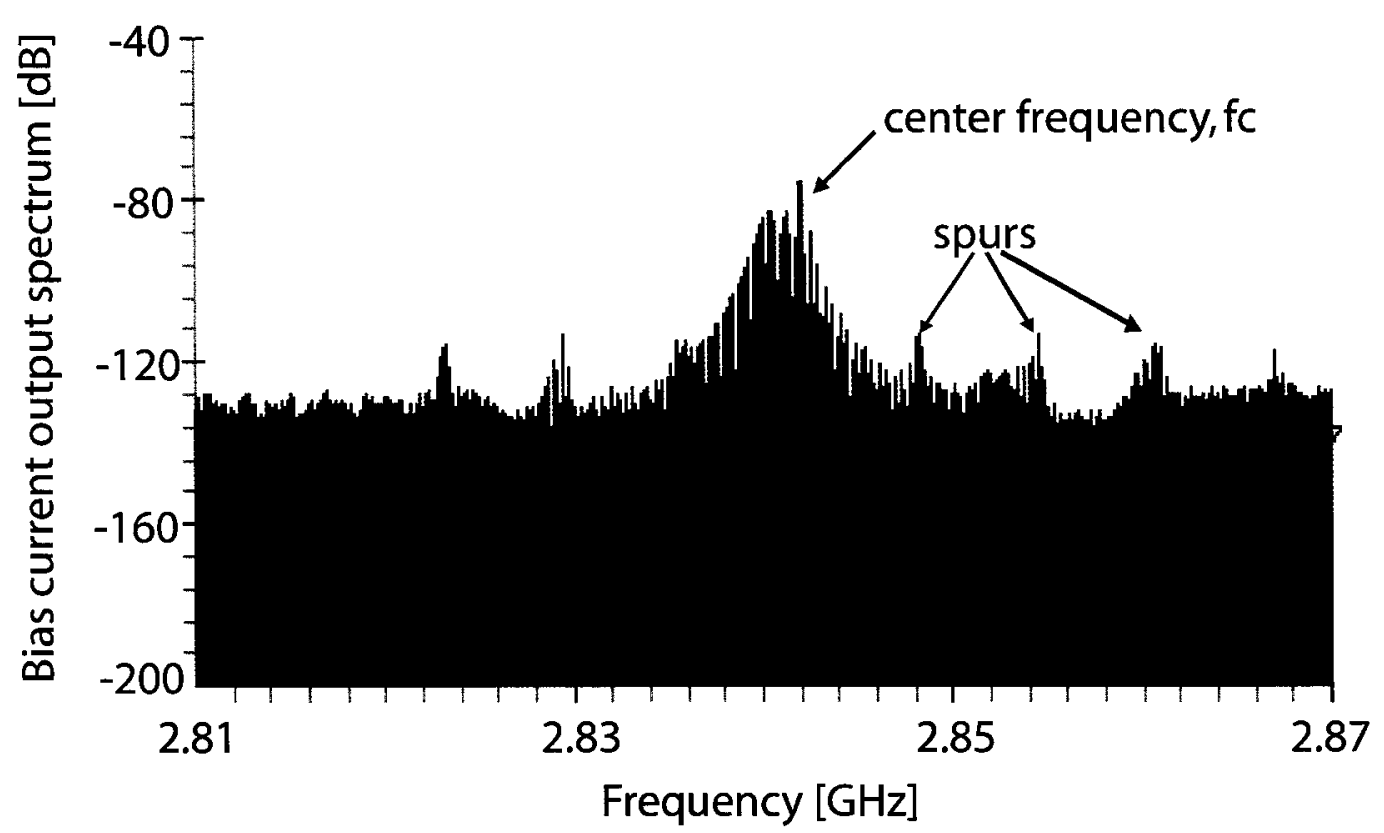

Figure 4.19: A zoomed in view of the spectrum of the bias current for the transient with noise simulation. The current waveform was transformed using a DFT with a bin size of $10 \mathrm{kHz}$, using 524,288 sample points. The clock was toggled at a rate of $1 \mathrm{MHz}$. Spurs due to the clock are seen in the spectrum plot.

In both cases, the VCO output spectrum was plotted using a DFT function, with a bin size of $10 \mathrm{kHz}$ and 524,288 sample points as is shown in Figure 4.21.

It is seen from Figure 4.21 that the switch causes a shift in the center frequency from $1.421 \mathrm{GHz}$ in the case where there is no switch in the VCO to $1.426 \mathrm{GHz}$ when the switch has a clock frequency of $1 \mathrm{MHz}$. As well, it is observed that there is a decrease in the amplitude of the center frequency, and an increase in the spreading of the spectrum near the center frequency for the plot in Figure 4.21(a) which is the VCO with the switch, when compared to spectrum in Figure 4.21(b), which is narrower. This spreading of the spectrum results in higher phase noise. Lastly, as was observed for the bias current noise, spurs due to the clock frequency can be observed in Figure 4.21(a) at offsets from the center frequency. These spurs will be discussed further in Section 4.8. Therefore the addition of the switch does result in a change in the output spectrum and this explains the $0.3 \mathrm{~dB}$ increase in phase noise that was observed in Section 4.5 when the switched-bias circuit was included in the VCO. 

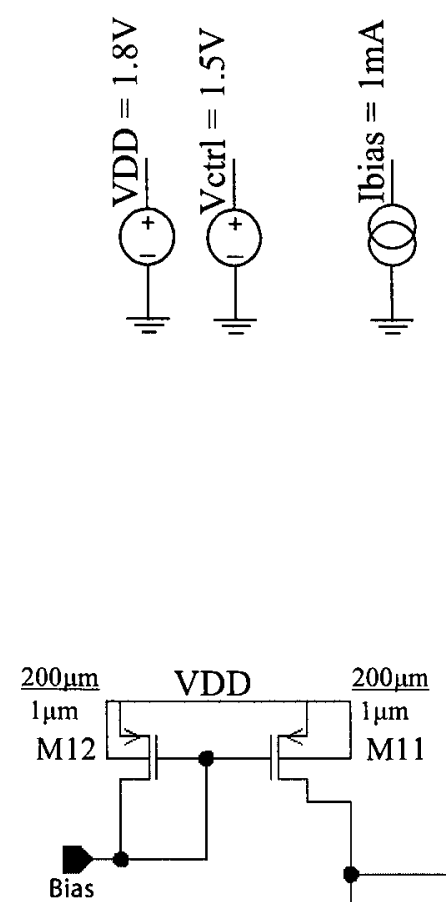

point (Ibias)

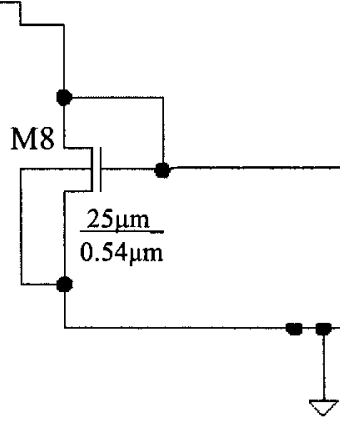

Figure 4.20: Testbench used to measure VCO output when the VCO does not have a switch. The transient simulation was run for $1 \mathrm{~ms}$, and the VCO output waveform was then transformed into its equivalent frequency spectrum using a DFT with a resolution of $10 \mathrm{kHz}$, using 524,288 sample points. 


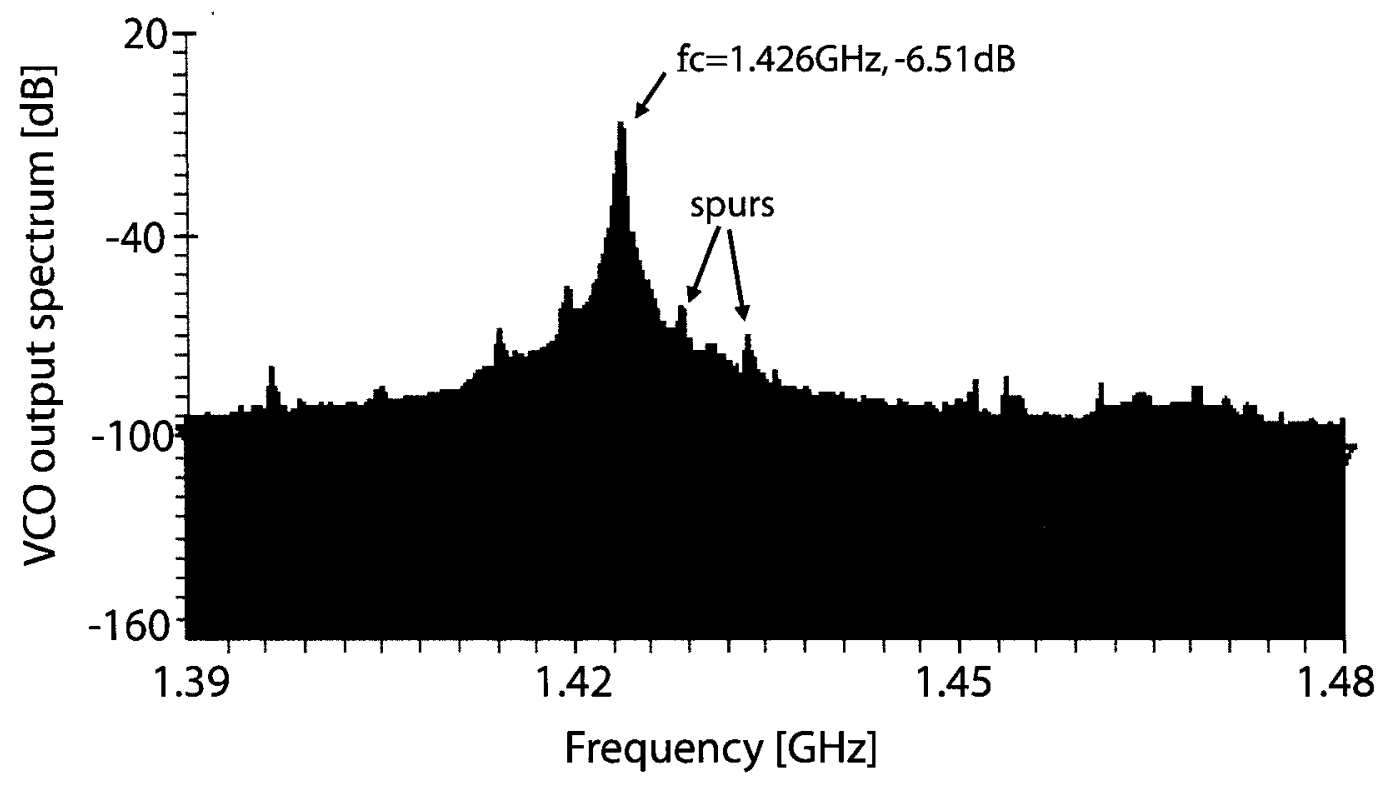

(a) Transient with noise and $1 \mathrm{MHz}$ clock switching

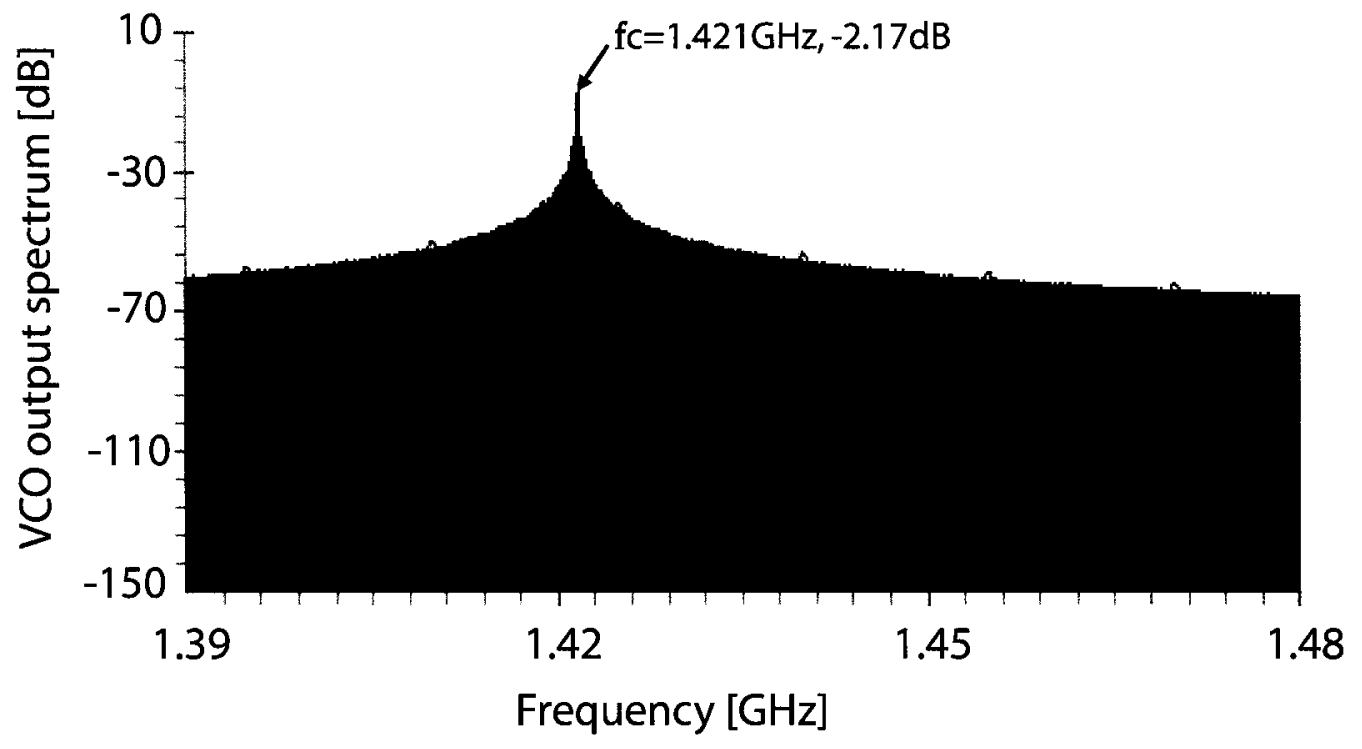

(b) Transient with no switch included in the VCO

Figure 4.21: The VCO output spectrum is plotted for the case: a) a transient noise simulation with a clock frequency of $1 \mathrm{MHz}$ toggling the switch, and b) a transient simulation for the VCO without a switch. The VCO output waveforms were transformed using DFTs with a bin size of $10 \mathrm{kHz}$, and 524,288 sample points. Spurs and spreading of the spectrum due to the clock are seen in the spectrum plot for (a) when compared to the spectrum in (b). 
Therefore this section has illustrated the impact that the noise in the bias current and the addition of the switch to the VCO circuit will have on the VCO phase noise. It has been shown that the noise contribution due to the switched-bias circuit leads to relatively small phase noise, and as a result should not have a large impact on the overall VCO phase noise. The next section will investigate the spot flicker and $\mathrm{kT} / \mathrm{C}$ noise values for the switched-bias circuit.

\subsubsection{Spot Noise}

It is also of interest to investigate which noise source, the flicker noise or the $\mathrm{kT} / \mathrm{C}$ noise, is larger at the frequencies of interest (i.e. at the close-in frequencies). This question is answered by plotting the spot flicker noise and the spot kT/C noise versus frequency, $\mathrm{f}$. The equation for the spot flicker noise is,

$$
S_{f l i c k e r}(f)=\frac{1}{1+\left(2 \pi f R_{o n} C\right)^{2}} \frac{k_{f}}{W L C_{o x} f}
$$

where $\mathrm{C}_{o x}$ is the gate capacitance per unit area, $\mathrm{W}$ and $\mathrm{L}$ are, respectively, the width and length of the switch transistor, $\mathrm{R}_{\text {on }}$ is the on resistance of the switch transistor, $\mathrm{C}$ is the bias retention capacitor, and $\mathrm{k}_{f}$ is the flicker noise process-dependent constant.

The equation for the spot $\mathrm{kT} / \mathrm{C}$ noise is derived by Kundert in [47],

$$
\begin{aligned}
& S_{c}(f)=\frac{4 m k_{b} T R_{o n}}{1+\left(2 \pi f R_{o n} C\right)^{2}}+\left[(1-m) \operatorname{sinc}\left(\frac{f}{f_{s}}(1-m)\right)\right]^{2} \frac{2 k_{b} T}{C f_{s}} \\
& S_{c}(f)=\frac{4 m k_{b} T R_{o n}}{1+\left(2 \pi f R_{o n} C\right)^{2}}+\left[(1-m) \frac{\sin \left(\pi \frac{f}{f_{s}}(1-m)\right)}{\pi \frac{f}{f s}(1-m)}\right]^{2} \frac{2 k_{b} T}{C f_{s}}
\end{aligned}
$$

where $k_{b}$ is Boltzmann's constant, $\mathrm{T}$ is the temperature in Kelvins, $\mathrm{R}_{o n}$ is the on resistance of the switch transistor, $C$ is the bias retention capacitor, $m$ is the duty cycle, $f_{s}$ is the sampling (i.e. clock) frequency and $\operatorname{sinc}(\mathrm{x})=\sin (\pi \mathrm{x}) /(\pi \mathrm{x})$.

The values of the parameters used to evaluate (4.7.13) and (4.7.14) are given in Table 4.5 , and the plot of the spot flicker and $\mathrm{k}^{\prime} \mathrm{T} / \mathrm{C}$ noises versus frequency is shown in Figure 4.22 . 
Table 4.5: Summary of parameters used to evaluate the noise due to the switched bias circuit

\begin{tabular}{|c||c|}
\hline $\mathrm{C}_{o x}$ & $8.46 \times 10^{-3} \mathrm{Fm}^{-2}$ \\
\hline $\mathrm{C}$ & $10 \mathrm{pF}$ \\
\hline $\mathrm{W}$ & $10 \mu \mathrm{m}$ \\
\hline $\mathrm{L}$ & $0.38 \mu \mathrm{m}$ \\
\hline $\mathrm{R}_{o n}$ & $437.2 \Omega$ \\
\hline $\mathrm{k}_{f}$ & $4.0 \times 10^{-25} \mathrm{~V}^{2} \mathrm{~F}$ \\
\hline $\mathrm{k}_{b}$ & $1.38 \times 10^{-23} \mathrm{~m}^{2} \mathrm{kgs}^{-2} \mathrm{~K}^{-1}$ \\
\hline $\mathrm{T}$ & 300 degrees Kelvin \\
\hline $\mathrm{m}$ & 0.5 \\
\hline $\mathrm{f}_{s}$ & $1 \mathrm{MHz}$ \\
\hline
\end{tabular}

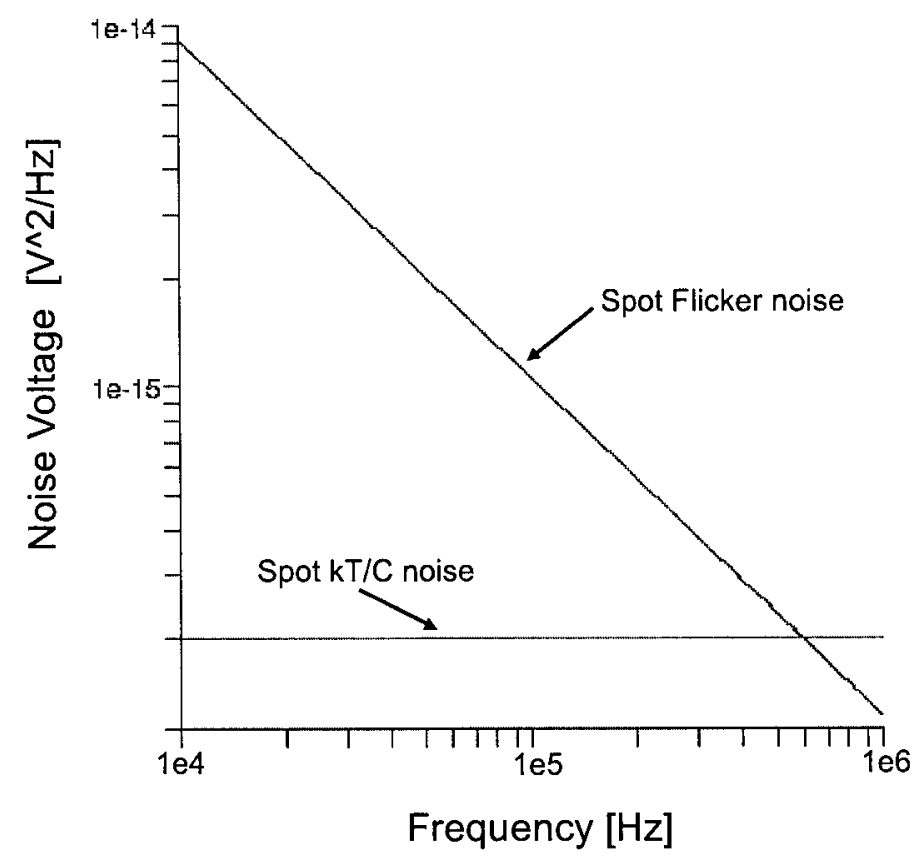

Figure 4.22: Plot of the spot flicker noise and the spot $\mathrm{kT} / \mathrm{C}$ noise versus frequency. It is seen that the flicker noise dominates at the lower frequencies. 
As can be seen from Figure 4.22, flicker noise is the dominate noise source at the lower frequencies. Therefore, for the close-in frequencies of interest for this research, the flicker noise will dominate over the $\mathrm{kT} / \mathrm{C}$ and therefore the flicker noise is the main noise source of interest. In addition, Figure 4.22 also indicates that the switching noise will not have a large impact on the overall phase noise as the flicker noise is several magnitudes larger.

\subsection{Spur Simulations}

The spurs that result due to the switching nature of switched-bias circuit will now be investigated. At the frequencies of interest the output signal at the gate of the tail current transistor $(\mathrm{M} 7)$ can be represented as a piecewise linear function, as shown in Figure 4.23. As shown in this figure, the signal is made up of a square wave portion which results from the square wave clock signal, a portion that resembles a sawtooth wave, which is a result of the droop of the gate voltage due to charge leakage from the bias retention capacitor, and a train of impulses that represent the spikes at the points when the clock is switching. Figure 4.23 shows a plot of the piecewise linear model, and a plot of the simulated actual voltage at the gate of M7 when the switch is toggled at a rate of $1 \mathrm{MHz}$. A comparison of these two plots shows that the approximation of the voltage at the gate of M7 using a piecewise linear model is valid and is a good approximation to the actual signal. In addition, the plots show the same shape as was observed in the measurement results of the change in voltage at the bias retention capacitor shown in Figure 5.15 in the measurement section (subsection 5.2.3) 


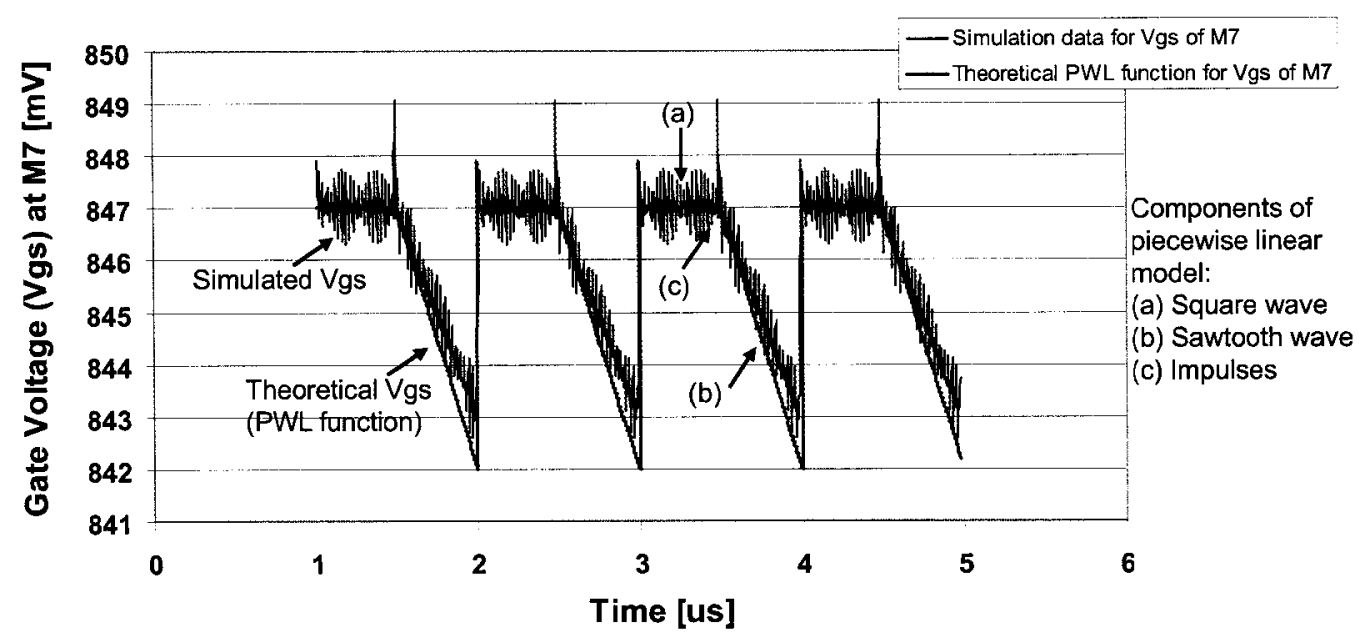

Figure 4.23: A comparison of the simulated Vgs voltage at the gate of M7 for a clock frequency of $1 \mathrm{MHz}$, and the piecewise linear model that is being used to represent the Vgs voltage at the gate of M7 to determine the location of spurs. This figure shows that the piecewise linear model is a good approximation for the frequencies of interest.

The equation used to obtain the theoretical plot is,

$$
V_{g s, p w l}=\frac{4}{\pi} \sum_{k=1}^{\infty} \frac{\sin \left((2 k-1) \omega_{c l k} t\right)}{(2 k-1)}+\frac{2}{\pi}\left(\frac{i_{l e a k}}{C f_{c l k}}\right) \sum_{k=1}^{\infty} \frac{\sin \left(k \omega_{c l k} t\right)}{k}+f_{c l k} \sum_{k=1}^{\infty} e^{j 2 \pi k f_{c l k} t}+V_{b i a s}
$$

where the first term is the Fourier series of the square wave due to the square wave clock; the second term is the Fourier series of a sawtooth waveform, multiplied by the rate of charge leakage $\left(\mathrm{i}_{\text {leak }}\right)$ of the bias retention capacitor, $\mathrm{C}$, which indicates the droop due to leakage; the third term is the Fourier series of a train of impulses which represent the noise at the switching points; and the last term is the DC bias voltage for the tail current transistor, M7.

To simulate the effect of spurs on the VCO output signal, a piecewise linear input source was used at the gate of the tail current transistor, M7, as shown in the testbench in Figure 4.24. 

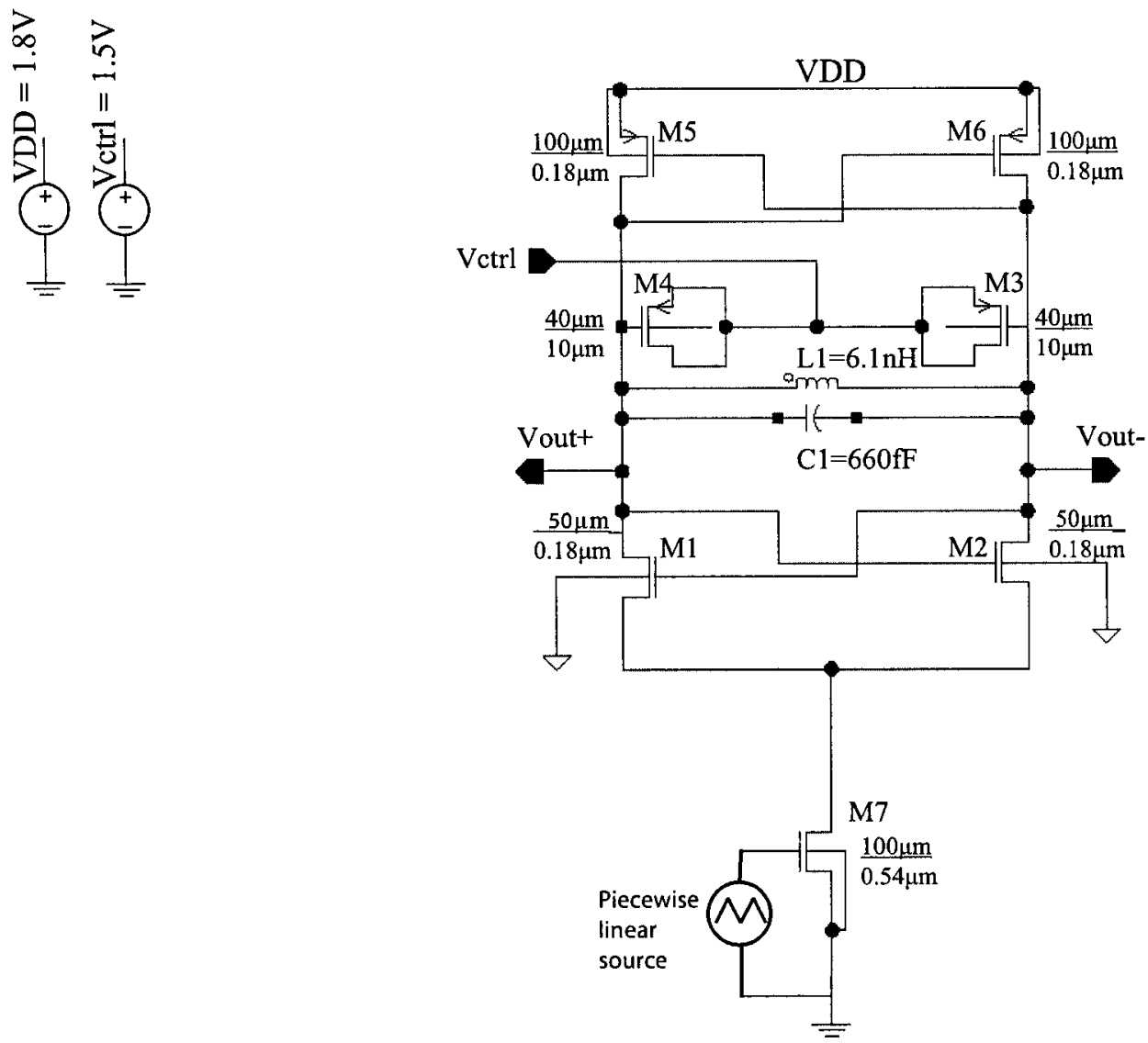

Figure 4.24: Testbench used to simulate the effect of spurs using transient simulations and a piecewise linear input source at the gate of M7, the NMOS transistor in the bias network forming the tail current of the VCO. 
To determine the expected location of the spurs, the piecewise linear expression in (4.8.1) can be expanded,

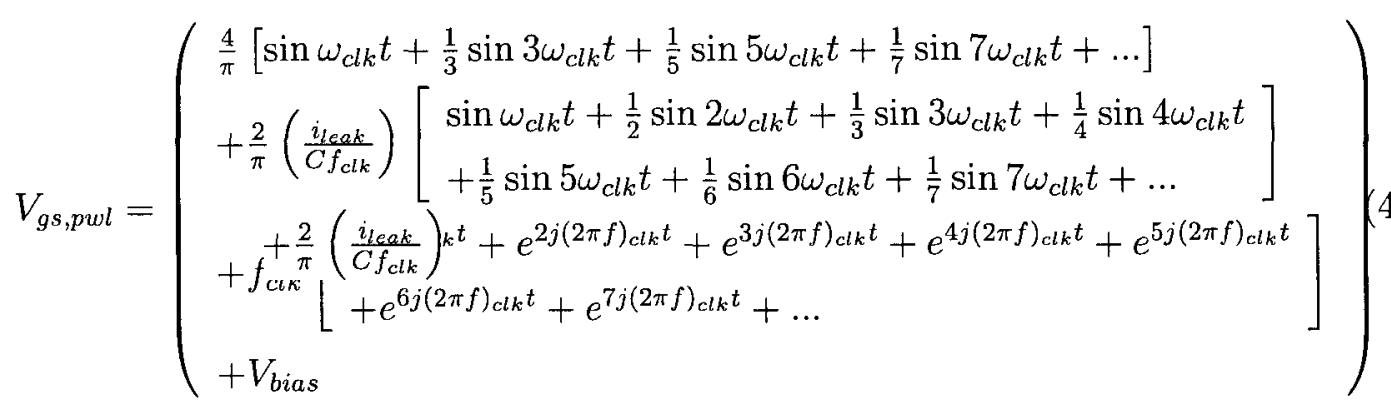

Therefore from (4.8.2) it is expected that the largest spurs will occur at the odd harmonics of the clock frequency (at offsets from the center frequency of the VCO), as at these frequencies the sawtooth waveform and the square waveform will be added together. There will also be smaller spurs occurring at the even harmonics due to the sawtooth waveform. All of the spurs will decrease in size as the frequency increases as the coefficients decrease for higher harmonics. The plot shown in Figure 4.25 is the frequency spectrum of the VCO output, and as expected, the spurs occur at offsets from the center frequency at the even and odd harmonics of the sampling frequency. Also, as was predicted, the odd harmonics have a larger amplitude than the even harmonics, and all of the spurs decrease in size as the frequency increases (as the offset from the carrier frequency increases).

Some of the factors that will affect the location and amplitude of the spurs include the clock amplitude and mismatch. As the clock amplitude increases, the size of the spurs will increase as the square waveform representing the clock in the piecewise linear model will increase and result in a larger contribution to the spurs. As for mismatch, the larger the mismatch, the more shift and spreading of the frequency spectrum and the spurs will spread out. Mismatch results in a shift in the center frequency of the VCO as the differential pair does not switch as well when there is mismatch.

The change of the voltage at the gate modelled by the piecewise linear model will result in a change in the bias current. The tail current transistor, M7, is generally in 


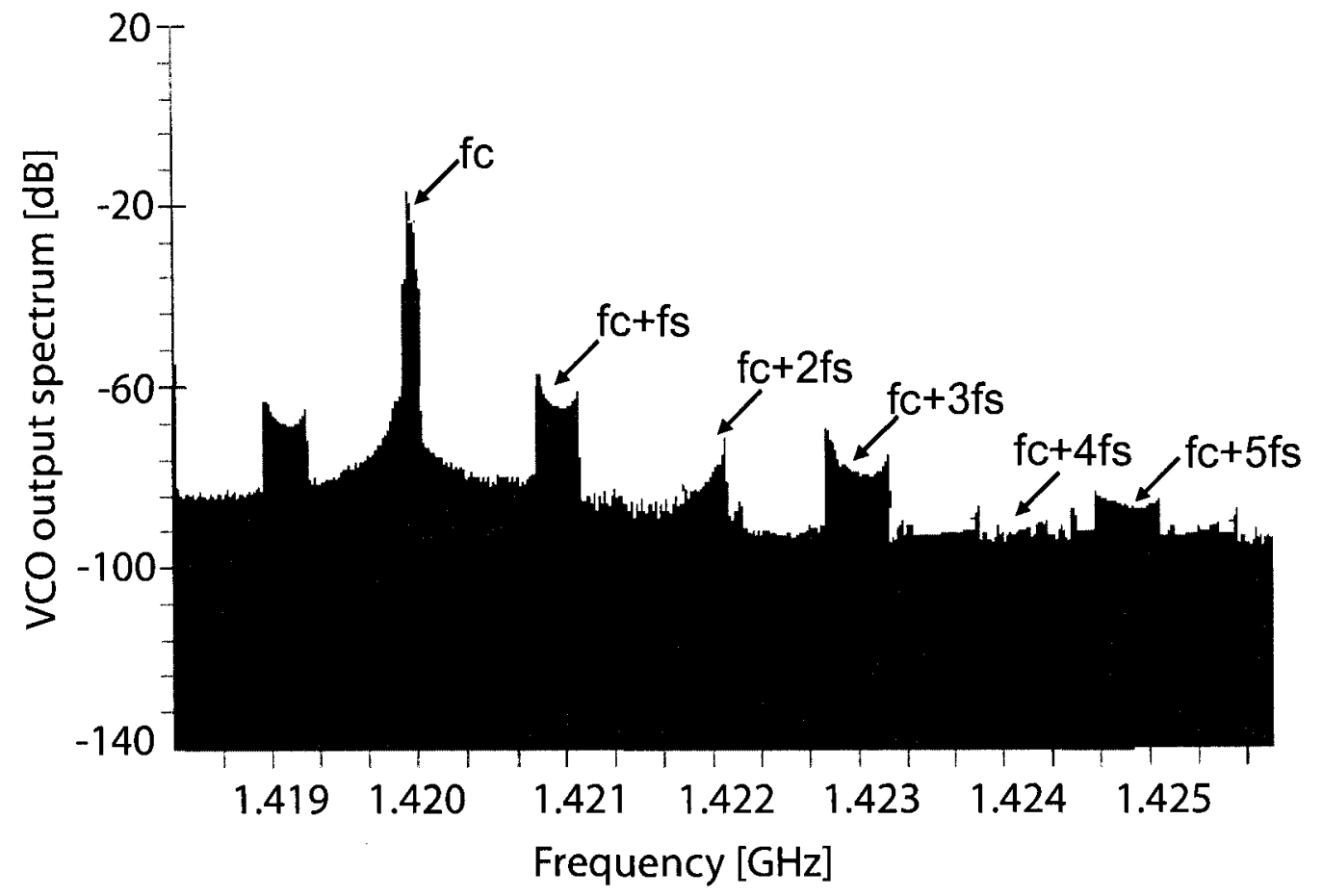

Figure 4.25: A DFT with a resolution of $10 \mathrm{kHz}$, done using 524,288 sample points was done on the transient VCO output when the input to the gate of the tail current transistor, M7, was a piecewise linear source. The transient simulation was run for $1 \mathrm{~ms}$. The result is spurs occurring at offsets from the center frequency, fc, at the harmonics of the sampling frequency, fs, of $1 \mathrm{MHz}$. 
triode, therefore using the large scale MOSFET equations, the bias current is related to Vgs of M7 as follows,

$$
I_{D, M 7}=I_{b i a s}=\frac{\mu_{n} C_{o x} W}{L}\left(\left(V_{G S}-V_{t h}\right) V_{D S}-\frac{V_{D S}^{2}}{2}\right)
$$

and substituting in the expression for Vgs given in (4.8.1), the complete expression for the bias current is,

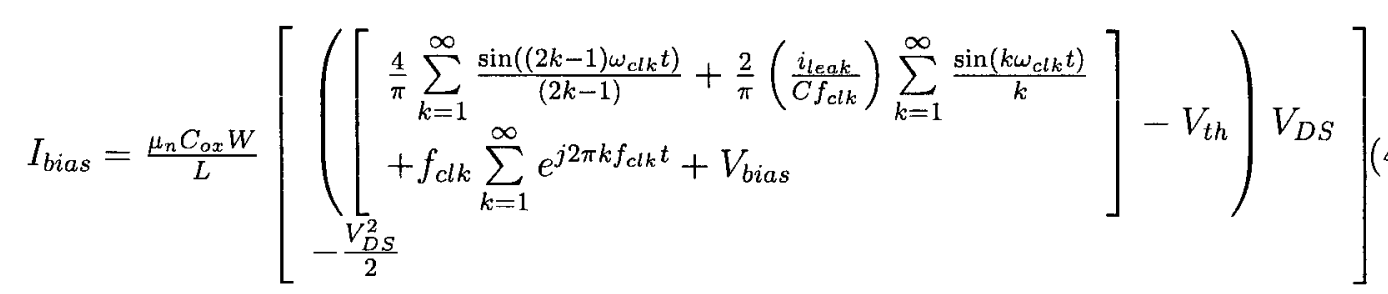

Therefore the change in Vgs for M7 will result in variations in the bias current. These variations in bias current may have an impact on the frequency of the VCO.

\subsection{Flicker Noise: Slope of Phase Noise Plot}

To illustrate that the flicker noise dominates in this circuit for close-in frequencies, the phase noise for the VCO both with the switch ON and OFF was plotted for offsets between $10 \mathrm{kHz}$ and $10 \mathrm{GHz}$, as can be seen in Figure 4.26. The slope per decade of the phase noise plot was calculated, and is shown on Figure 4.26. As was mentioned in subsection 2.6.1, for the frequencies where the flicker noise dominates, there should be a slope of $30 \mathrm{~dB} /$ decade. At the $1 / \mathrm{f}$ noise corner of the circuit, the slope becomes $20 \mathrm{~dB} /$ decade, and then finally becomes relatively flat when the thermal noise dominates at the higher frequencies. Therefore, as expected, the flicker noise dominates up to an offset frequency of approximately $1 \mathrm{MHz}$, after which point the slope begins to decrease towards $20 \mathrm{~dB} /$ decade. With this decrease in the slope it is also observed that the reduction in phase noise observed between the case when the switch is opened and when the switch is closed begins to decrease, again reinforcing the idea that the switched-bias technique is most useful at the close-in frequencies. 


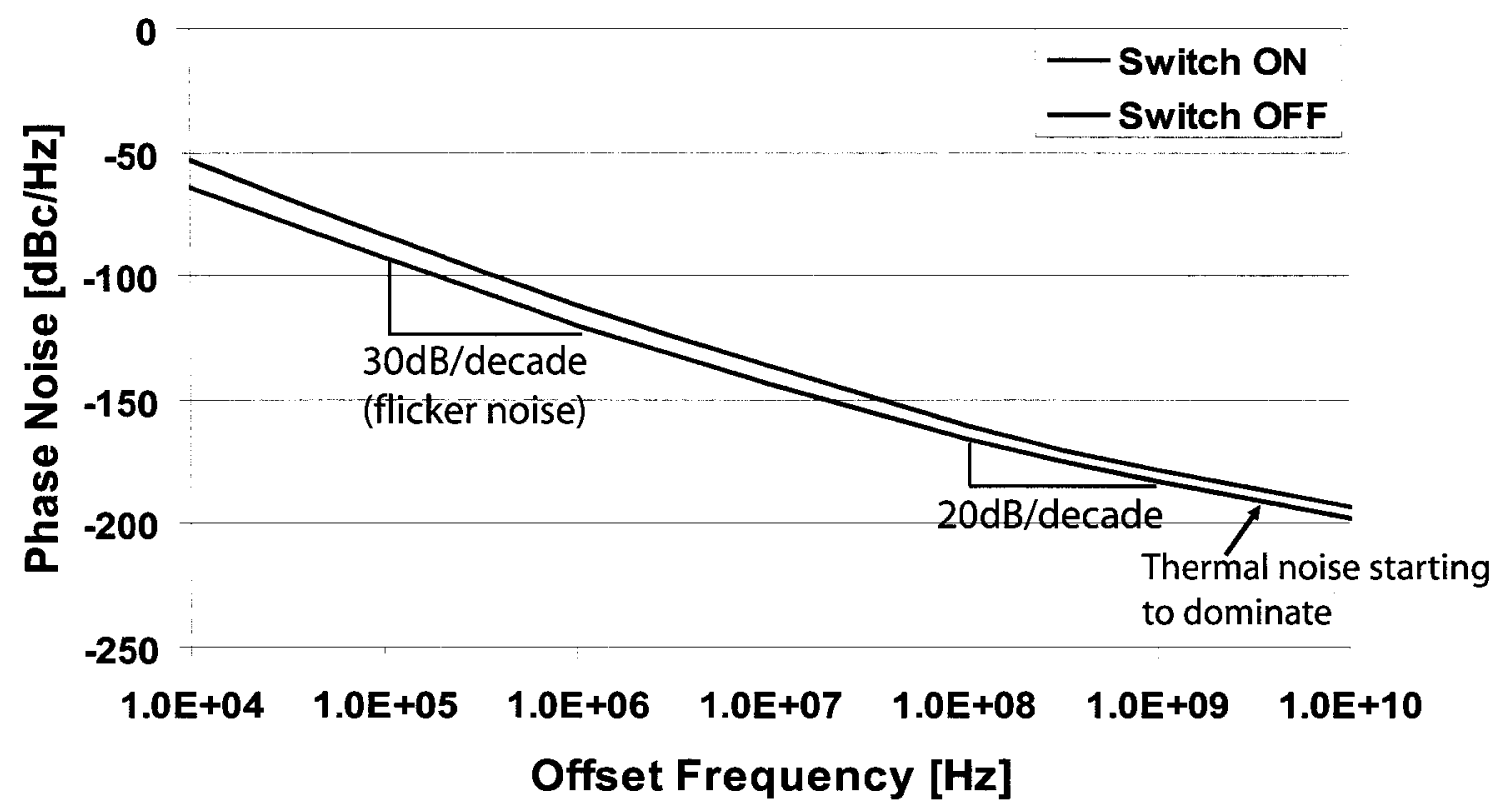

Figure 4.26: The slope of the phase noise plot is shown for offset frequencies between $10 \mathrm{kHz}$ and $10 \mathrm{GHz}$. The flicker noise is shown to dominate up to approximately $1 \mathrm{MHz}$.

\subsection{Chapter Summary}

- This chapter began with a look at the design of the VCO presented in $[8$, pp.1240]. The simulated and measured results for this VCO, which was used as the benchmark for this research in terms of phase noise values, were also presented. The bias network of the VCO presented in [8, pp.12-40] was modified to include the switched-bias circuit.

- Next the focus moved to the integration of the switched-bias circuit into the VCO.

- The layout for the complete circuit was presented, including a discussion on the particular layout practices that were taken into consideration.

- In addition, a discussion of the simulation challenges were presented. Lastly, the simulation results themselves were presented to validate the use of the switched-bias circuit within a VCO to reduce phase noise. This includes a discussion of the impact spurs that may result due to the toggling of the switch may have on the VCO. 


\section{Chapter 5}

\section{Measurement Results}

\subsection{PCB design}

A printed circuit board (PCB) was designed to test the manufactured chip. The die was mounted on a gold-plated landing pad using conductive silver epoxy. It is estimated that the bond wires are approximately, on average, $3 \mathrm{~mm}$ in length. The bond wires were used to connect the twenty-two aluminum pads on the integrated circuit to the appropriate traces on the PCB.

The PCB was designed by Vladimir Vukovic and the top and bottom views of the PCB layout showing the connections made between the chip and components are presented in Figure 5.1 and Figure 5.2, respectively. The PCB was fabricated by CMR Summit Technologies on a two-layer, 62-mil panel with an FR4 substrate. The PCB has a size of 3inches by 2 inches. The chip landing pad is $4 \mathrm{~mm}$ by $4 \mathrm{~mm}$, which was used to accommodate the overall chip size of $3 \mathrm{~mm}$ by $3 \mathrm{~mm}$. The two-sided board was chosen to allow signal routing on both sides of the board. The bottom of the PCB also provides a solid ground-plane underneath the output RF signal.

Both through-hole and surface mount components were used in this design. All of the components placed on the top side of the board except for the power-supply decoupling capacitors, which were placed on the bottom of the board due to space constraints. A complete bill of materials (BOM) is shown in Appendix A, and the photo of the populated PCB is shown in Figure 5.4. A combination of SMA connectors and pins were used to connect the inputs and outputs of the PCB to test equipment. A list of the signals the 
SMA connectors are used for is given in Table 5.1.

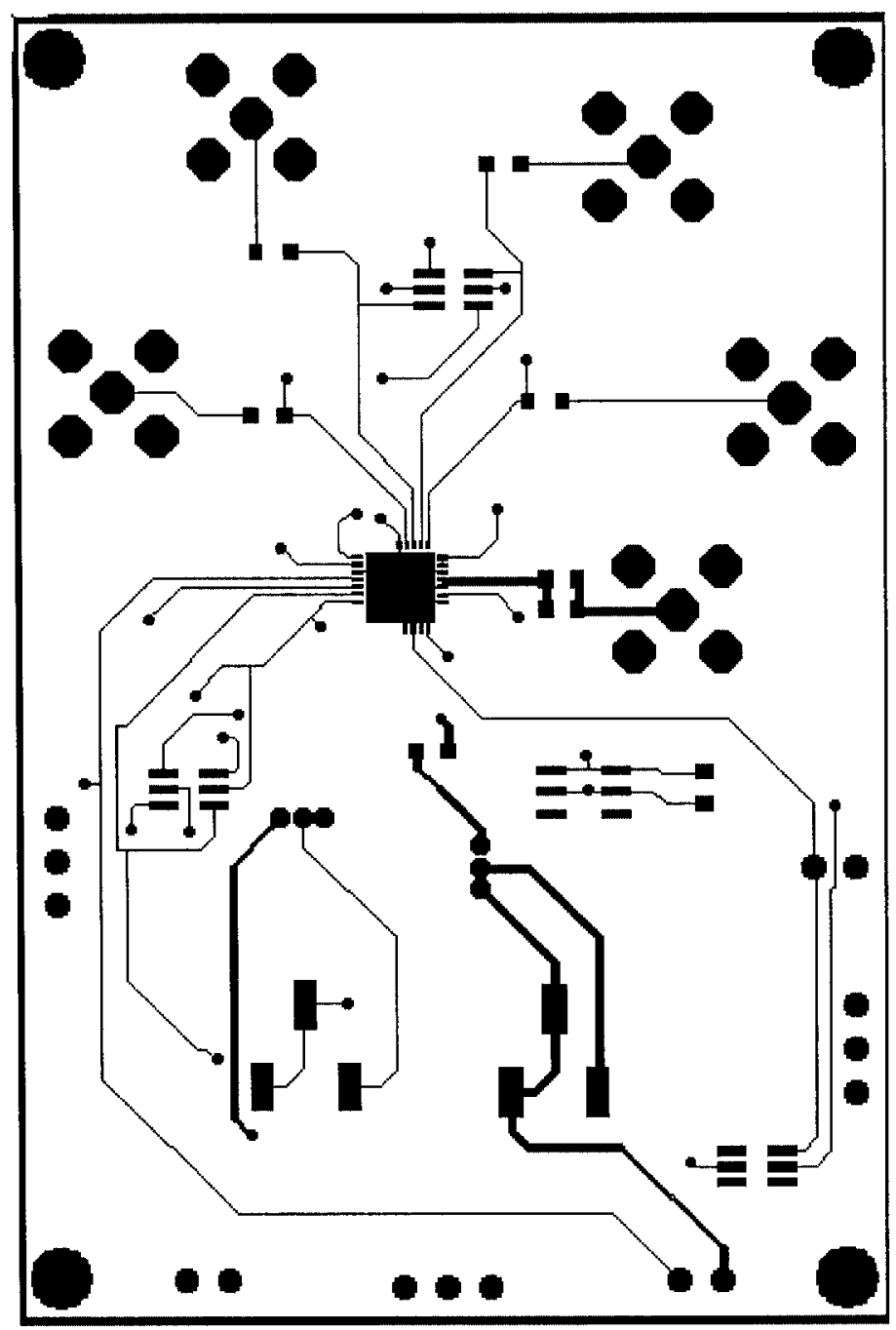

Figure 5.1: The top view of the PCB layout.

A block diagram showing an overview of the connections on the PCB to the fabricated chip, ICFCUCFD, is shown in Figure 5.3. These connections include the currents used to bias the VCO, the control voltage, Vctrl, used to tune the VCO, the clock inputs used to turn the main switch and dummy switch used in the switched-bias circuit on and off, the power supply connections, the bias test point, VcapTest, and lastly the output, Fout. The following sections will discuss how the power supply and DC signals, the current sources, the ESD protection, the output, and the bonding were implemented. 


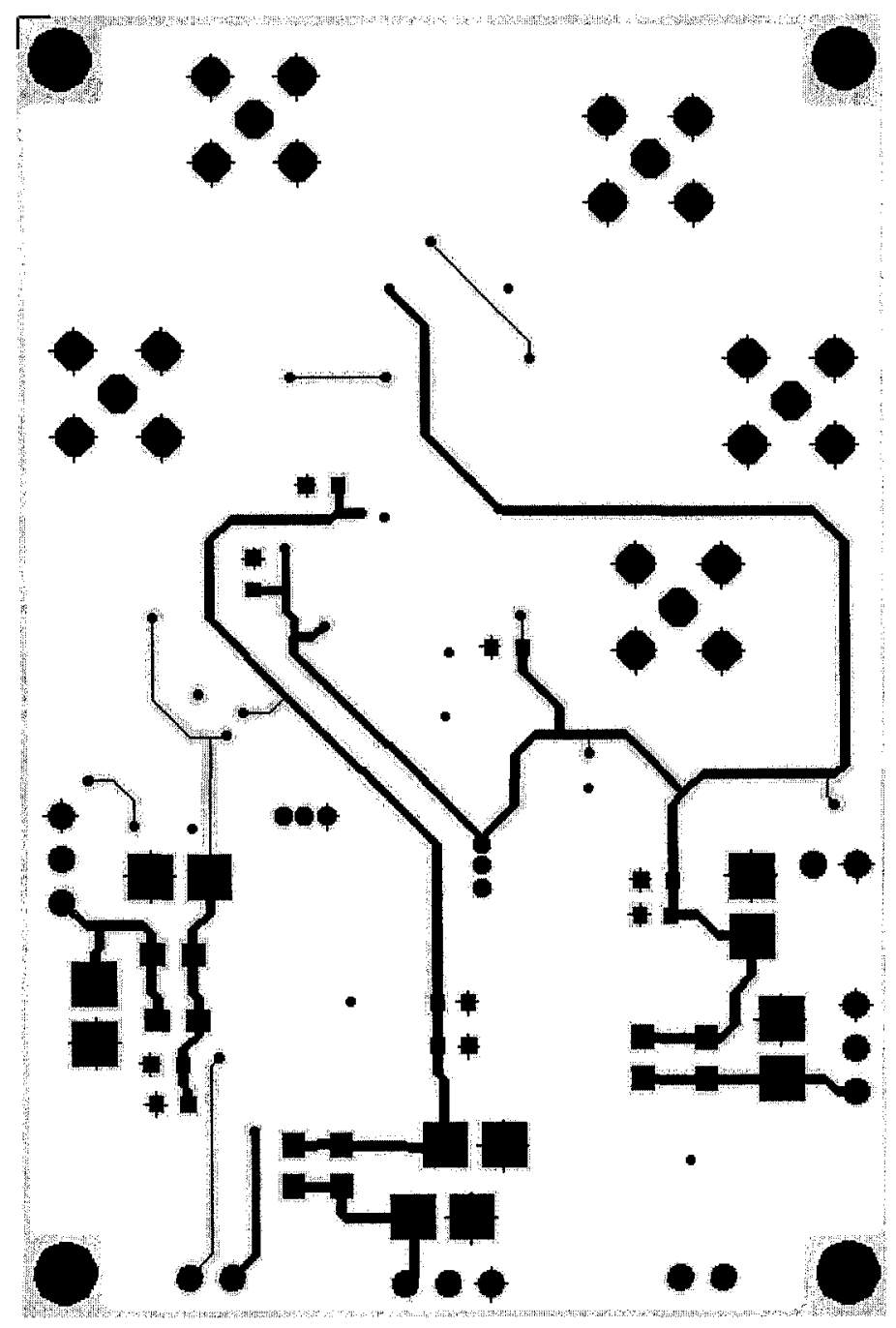

Figure 5.2: The bottom view of the PCB layout.

Table 5.1: List of the signals connected to the SMA connectors

\begin{tabular}{|c|c|c|}
\hline Number & Signal Name & Explanation of the signal \\
\hline SMA1 & SwitchCLK3bar & $\begin{array}{l}\text { Second clock input used to control the second } \\
\text { dummy switch }\end{array}$ \\
\hline SMA2 & SwitchCLK1bar & $\begin{array}{l}\text { First clock input used to control the first } \\
\text { dummy switch }\end{array}$ \\
\hline SMA3 & SwitchCLK1 & $\begin{array}{l}\text { First clock input used to control the first } \\
\text { main switch }\end{array}$ \\
\hline SMA4 & SwitchCLK3 & $\begin{array}{c}\text { Second clock input used to control the second } \\
\text { main switch }\end{array}$ \\
\hline SMA5 & Fout & Output of the VCO \\
\hline
\end{tabular}




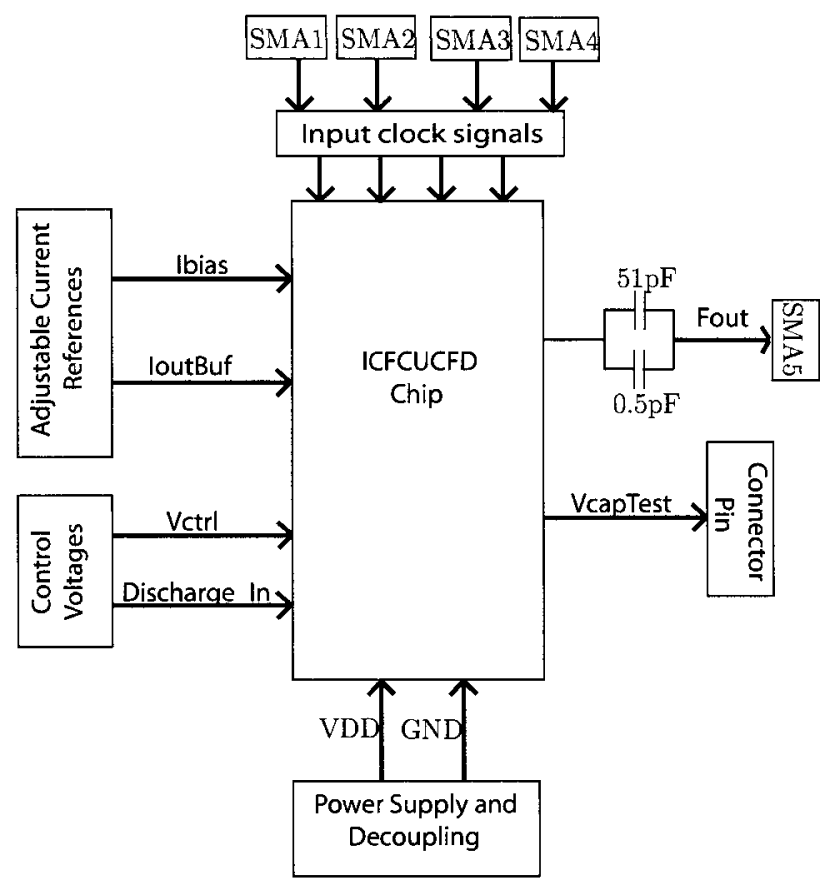

Figure 5.3: Block diagram showing the PCB layout.

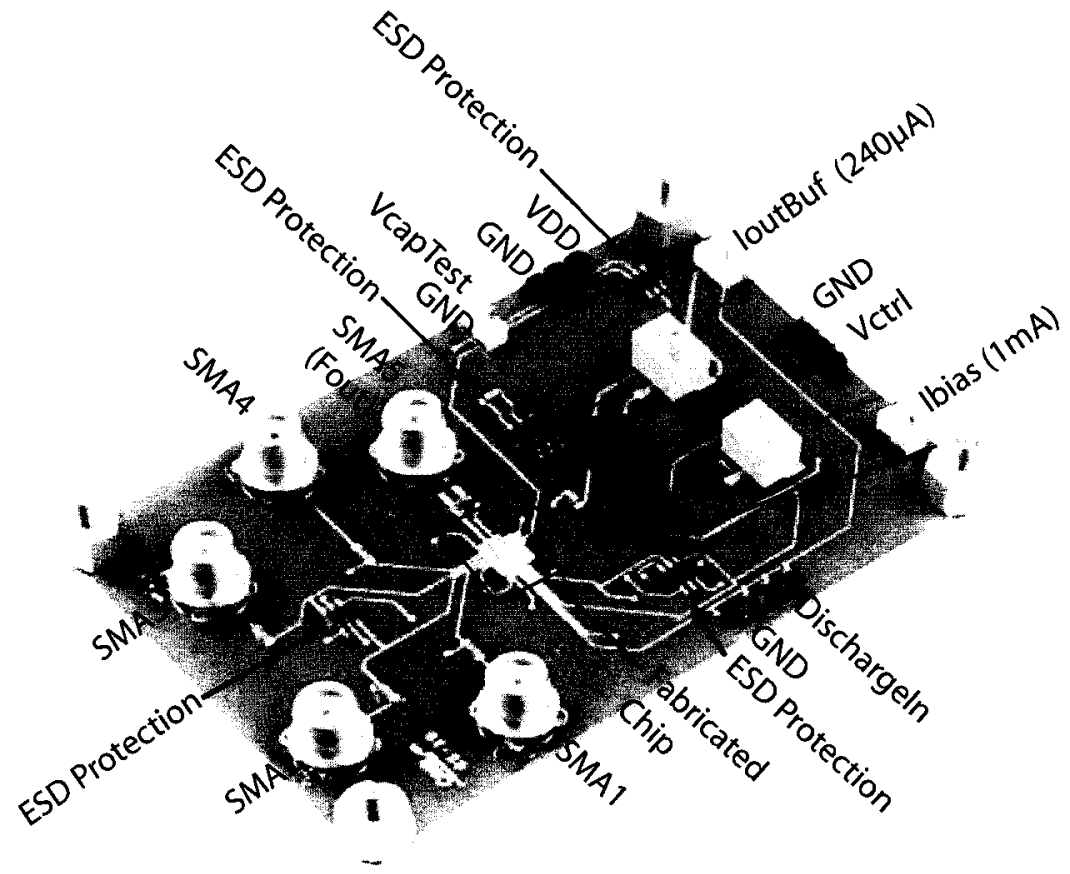

Figure 5.4: Photo of the populated PCB. 


\subsubsection{Power Supply and DC inputs}

A $1.8 \mathrm{~V}$ voltage supply was used to power this design. In addition, to achieve the $1.4 \mathrm{GHz}$ frequency for the VCO, a control voltage, Vctrl, of $1.5 \mathrm{~V}$ was applied to the circuit. The last DC signal to consider is the signal DischargeIn, which was described in subsection 4.2 as a signal used to provide a mechanism to discharge the bias retention capacitor during testing.

A decoupling network consisting of a ferrite bead and various size capacitors was placed on each DC input line to help noise that can result from noisy power supplies. The circuit used for the power supply decoupling is shown in Figure 5.5.

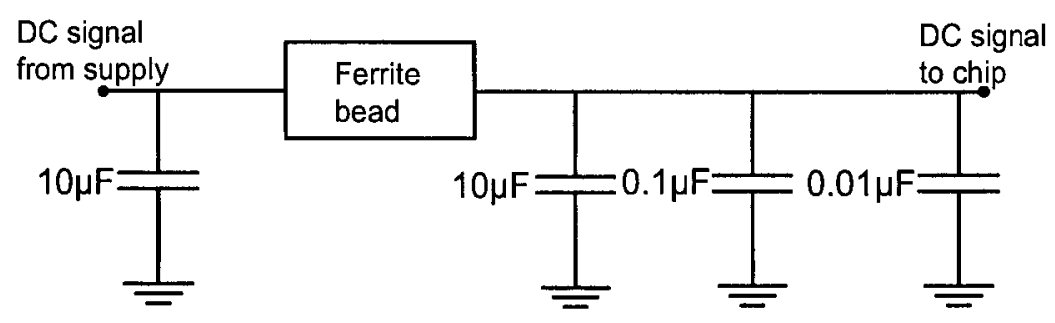

Figure 5.5: DC signal decoupling network.

The decoupling network uses bypass capacitors with values of $10 \mu \mathrm{F}, 0.1 \mu \mathrm{F}$ and $0.01 \mu \mathrm{F}$, as suggested in [49], to present low-impedance paths which help reduce noise. This range of capacitors was chosen to help filter both low and high frequency noise. The presence of the ferrite bead provides a large series impedance path to ground for unwanted AC signals, while still providing a low impedance at DC.

\subsubsection{Current Sources}

The fabricated chip requires two bias currents, one for the bias network and one for the output buffer. Current sources on the PCB were used to generate the current the Ibias, the current used to bias the VCO, and Ioutbuf, the current used to bias the output buffer. The circuitry to generate these current is shown in Figure 5.6. The figure shows that the configuration to generate Ibias (see Figure 5.6(a)) and Ioutbuf (see Figure 5.6(b)) are different. This is due to the fact that Ibias is the current for a PMOS current mirror, 
while Ioutbuf is the current for an NMOS current mirror.

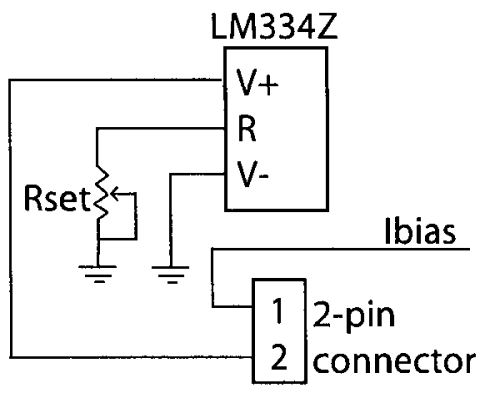

(a)

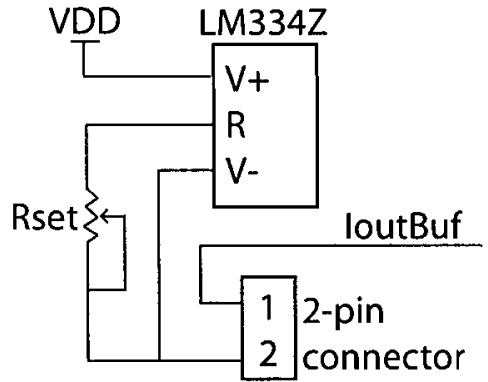

(b)

Figure 5.6: Current reference configurations used to source/sink current in the current mirrors.

The current is controlled through the use of a three-terminal adjustable current source (part number: LM334Z). LM334Z is a current source chip that generates a current that can be controlled by changing the resistance across $\mathrm{R}$ and $\mathrm{V}$ - terminals, as shown in Figure 5.6, though the use of a potentiometer (represented by $\mathrm{R}_{S E T}$ in Figure 5.6). The relationship between current generated and the resistance providing in [50] is given by equation,

$$
I_{R E F}=\frac{227 \mu V /{ }^{\circ} \mathrm{K}}{R_{S E T}}=\frac{68 m V}{R_{S E T}}
$$

where $\mathrm{I}_{R E F}$ is the current generated by the source and $\mathrm{R}_{S E T}$ is the resistance between the $\mathrm{R}$ and $\mathrm{V}$ - terminal. For a temperature of 298 degrees Kelvin (approximately room temperature and the expected operating temperature for the PCB), as shown, the numerator of (5.1.1) reduces to $68 \mathrm{mV}$.

Based on the minimum value of current required, the potentiometer size required to generate Ibias and Ioutbuf was determined using (5.1.1). The maximum resistance for each current generated is shown in Table 5.2.

A sample calculation for the values shown in Table 5.2 is shown by equation,

$$
R_{S E T}=\frac{68 m V}{I_{R E F}}=\frac{68 m V}{1 m A}=68 \Omega
$$


Table 5.2: Potentiometer resistance value and currents generated on the PCB

\begin{tabular}{|c||c|c|}
\hline Current name & Current value & Potentiometer (maximum) resistance \\
\hline Ibias & $1 \mathrm{~mA}$ & $100 \Omega$ \\
\hline Ioutbuf & $240 \mu \mathrm{m}$ & $1 \mathrm{k} \Omega$ \\
\hline
\end{tabular}

with $\mathrm{I}_{R E F}=$ Ibias set to $1 \mathrm{~mA}$. The result is a required resistance of $68 \Omega$, therefore the LM334Z current source with a maximum resistance of $100 \Omega$ was chosen to generate the current for Ibias. The same calculation was done to determine the resistance value required for Ioutbuf.

A 2-pin connector is used to allow the current to be measured and set to the appropriate value using a multimeter, as the potentiometer is adjusted. Once the desired current is achieved, a jumper is placed across the two pins of the connector, creating a path for the current to flow into the chip.

\subsubsection{ESD protection}

The ESD protection circuitry was added to all DC and low-frequency (i.e. the clock) inputs that are connected to the gate of a transistor. The ESD protection circuitry has been labelled on the photo of the PCB shown in Figure 5.4. This circuitry is used to protect the fabricated chip from large voltages that could damage the transistors. A diagram of the ESD protection circuitry, as presented in [35], is shown in Figure 5.7. As described in [35], the reverse-biased diodes are used to direct charge that results during an ESD event (ex. a large voltage could be accidently transferred to the chip while being handled if the person was not properly grounded) to the supply-rails and therefore away from the transistor on the chip.

During normal operation of the chip (i.e. no ESD event) the diodes are reverse biased and the signal is travels to the chip as expected. However, when an ESD event occurs, the diodes become forward biased, allow the excess charge to be re-directed to the PCB power supply network and away from susceptible transistors on chip. An additional zener diode was added between the supply line and ground to help further facilitate the dissipation of the charge to the ground plane. 
The ESD protection circuit is implemented using the NUP4301MR6T1 diode array component from ON Semiconductor [51] as the clamp, reverse-biased diodes. This diode array can simultaneously protect four data lines, which helped reduce the number of components required on the PCB. The zener diode is implemented using the DS9502 component from Dallas Semiconductor [52].

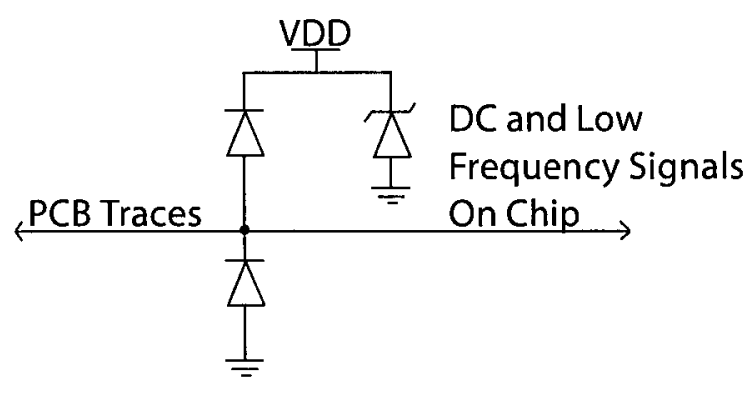

Figure 5.7: Schematic of the ESD (electrostatic discharge) protection circuitry.

\subsubsection{Bonding}

As mentioned above, the chip was mounted on a gold-plated landing pad using conductive silver epoxy. The bonding placement was done by Laurent Mouden using the facilities at École Polytechnique in Montreal. The bond wires are aluminum and have a $25 \mathrm{~m}$ diameter. The bonding diagram used to facilitate the bonding process by showing the connections between the chip and the PC is shown in Figure 5.8. The actual bonded chip is shown in Figure 5.9. As can be seen from the photo, care was taken to ensure the bond wires did not contact the landing pad, which would result in the chip being shorted out. It can also be observed that long bond wires were required to attach the DC signals, as it was required to jump over another circuit that was fabricated on the same chip. This again shows that the placement of the signals, as described in section 4.2 was important, as the DC signals can tolerate the longer bond wires better than the RF output would, due to the extra resistance associated with longer bond wires. 


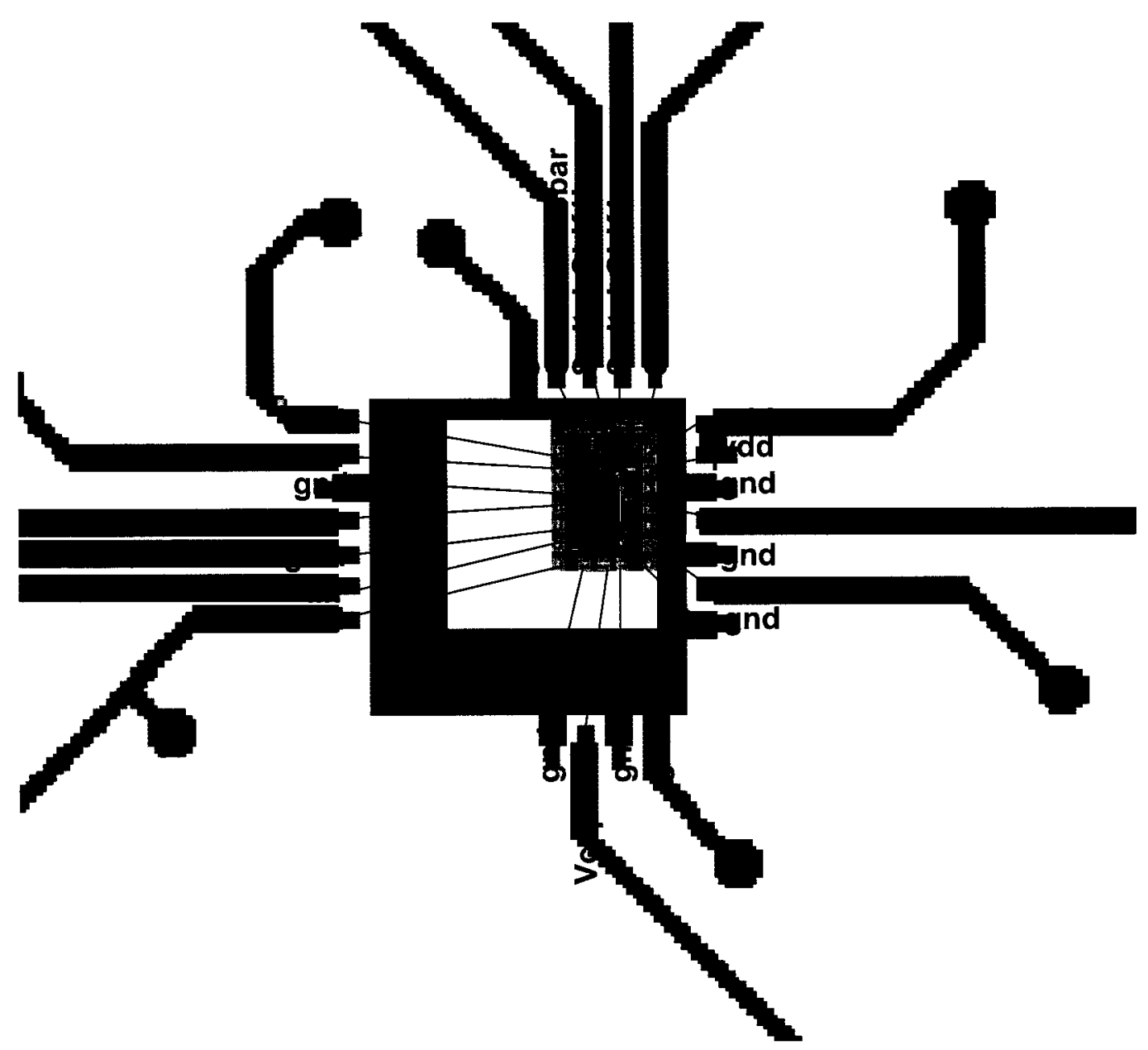

Figure 5.8: Bonding diagram used to indicate bond wire connections between the chip and the PCB. 


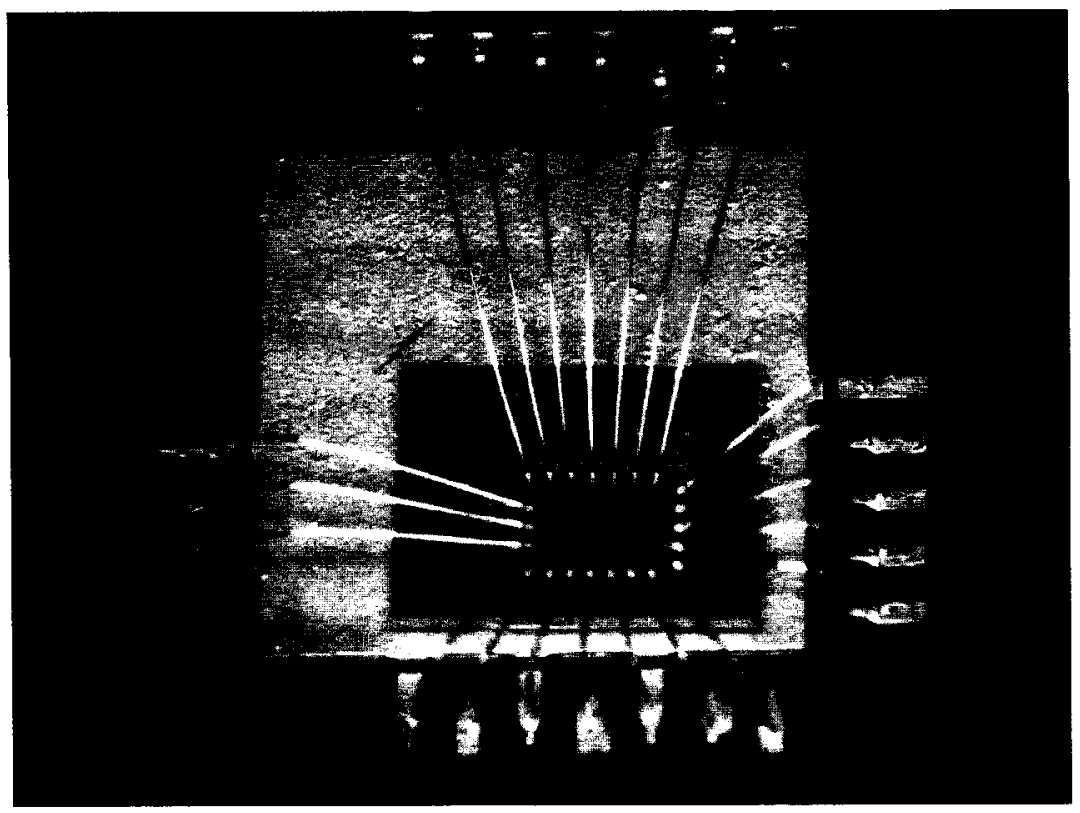

Figure 5.9: Photo of the chip bonded to the PCB.

\subsubsection{RF output}

The RF output of the chip was designed to be measured using either a spectrum analyzer or an oscilloscope. For that reason, the output trace on the PCB is connected to an SMA connector. DC blocking capacitors are placed along the output trace to block any DC components of the signal that may be present. A $51 \mathrm{pF}$ and $0.5 \mathrm{pF}$ capacitor were chosen as they had a self-resonant frequency at the frequency of interest, and the combination of the two capacitors provides a larger bandwidth than would be obtained with only one of these capacitors.

\subsection{Test setup and Measurement Results}

\subsubsection{Test Equipment}

The following equipment was used during the testing process:

- Agilent Infiniium 54855A DSO 6GHz Oscilloscope

- Agilent E4440A PSA Series Spectrum Analyzer, 3Hz-26.5GHz 
- Agilent 33250A 80MHz Function/Arbitrary Waveform Generator

- Agilent 6624A System DC Power Supply

- Keithley 2010 Multimeter

\subsubsection{Testing of VCO without Switched-Bias Circuit}

The version of the VCO which did not contain the switched-bias circuity was tested first to serve as a benchmark against which the VCO with the switched-bias circuit could be compared. A diagram of the test setup used to take the measurements is shown in Figure 5.10. It was determined that for a control voltage, Vctrl, of $1.5 \mathrm{~V}$, the center frequency for the VCO without the switched-bias circuit was $1.47 \mathrm{GHz}$, as is shown in the result obtained from the oscilloscope, Figure 5.11. The phase noise for the VCO was measured using the spectrum analyzer, as shown in Figure 5.12. A comparison between the simulated phase noise values presented in section 4.5 and the measured phase noise (PN) for the VCO without the switched-bias circuit is shown in Figure 5.13. The values match well at the lower offset frequencies, with a $1.6 \%$ difference between the simulated and measured phase noise for the VCO at a $100 \mathrm{kHz}$ offset. However, the difference in the values increases as the offset frequency increases. At an offset of $10 \mathrm{MHz}$, the difference between the simulated and measured phase noise values for the VCO rises to $8.5 \%$. This indicates that in reality the thermal noise floor is higher than was used in the simulations, as the thermal noise becomes the dominant noise at higher frequencies.

Comparisons between the simulation results presented in section 4.1 and section 4.5 and measured results for the VCO without the switched-bias circuit are shown in Table 5.3 .

Table 5.3: Comparison of simulated and measured results for the VCO without the switched-bias circuit

\begin{tabular}{|l||c|c|c|}
\hline Parameter & Simulated & Measured & Percentage Difference \\
\hline Center Frequency & $1.41 \mathrm{GHz}$ & $1.47 \mathrm{GHz}$ & $4.3 \%$ \\
\hline Tuning Range & $17 \%$ & $4.8 \%$ & $72 \%$ \\
\hline Power dissipation & $6.6 \mathrm{~mW}$ & $9.9 \mathrm{~mW}$ & $33 \%$ \\
\hline
\end{tabular}




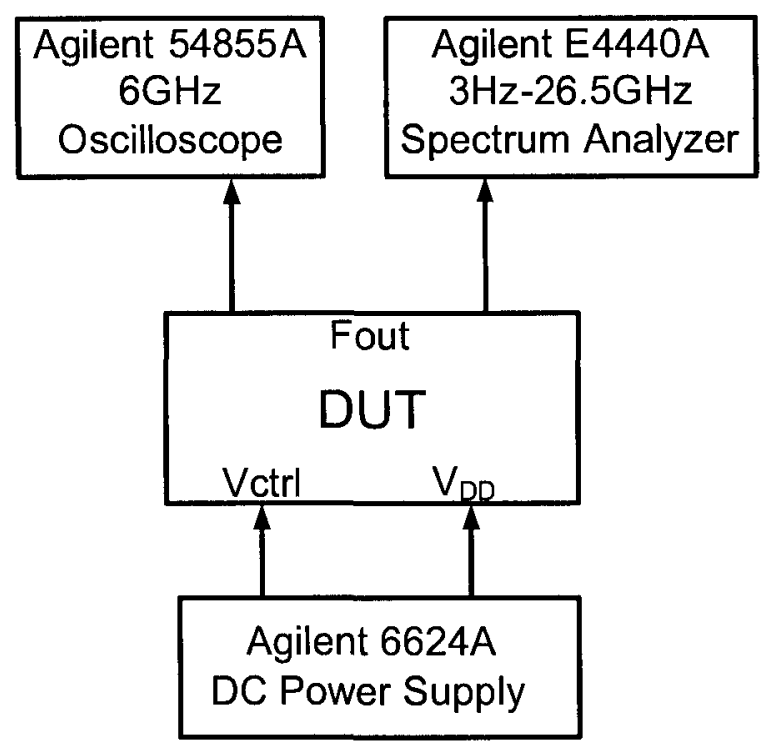

Figure 5.10: Test setup for the measurements taken for the VCO without the switchedbias circuit.

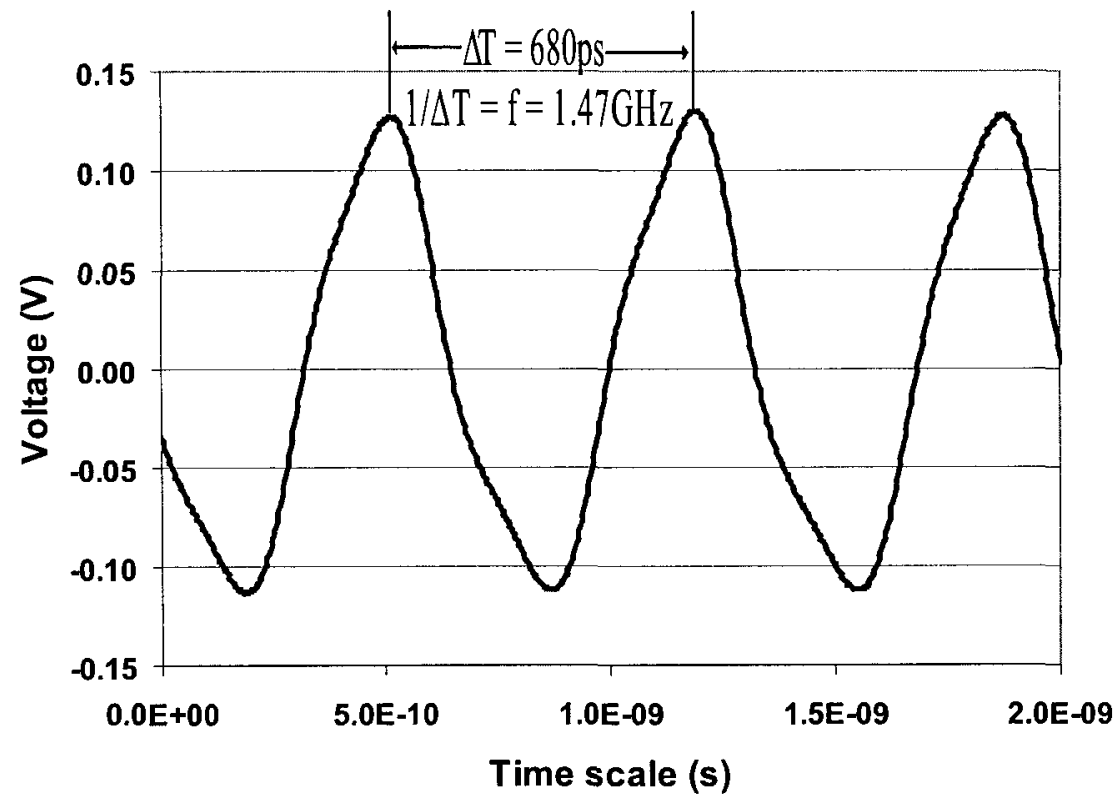

Figure 5.11: Plot of the output of the VCO without the switched-bias circuit from the oscilloscope. Calculations (inverse of the time period as shown in the plot) show that the center frequency of the oscillator is $1.47 \mathrm{GHz}$. 


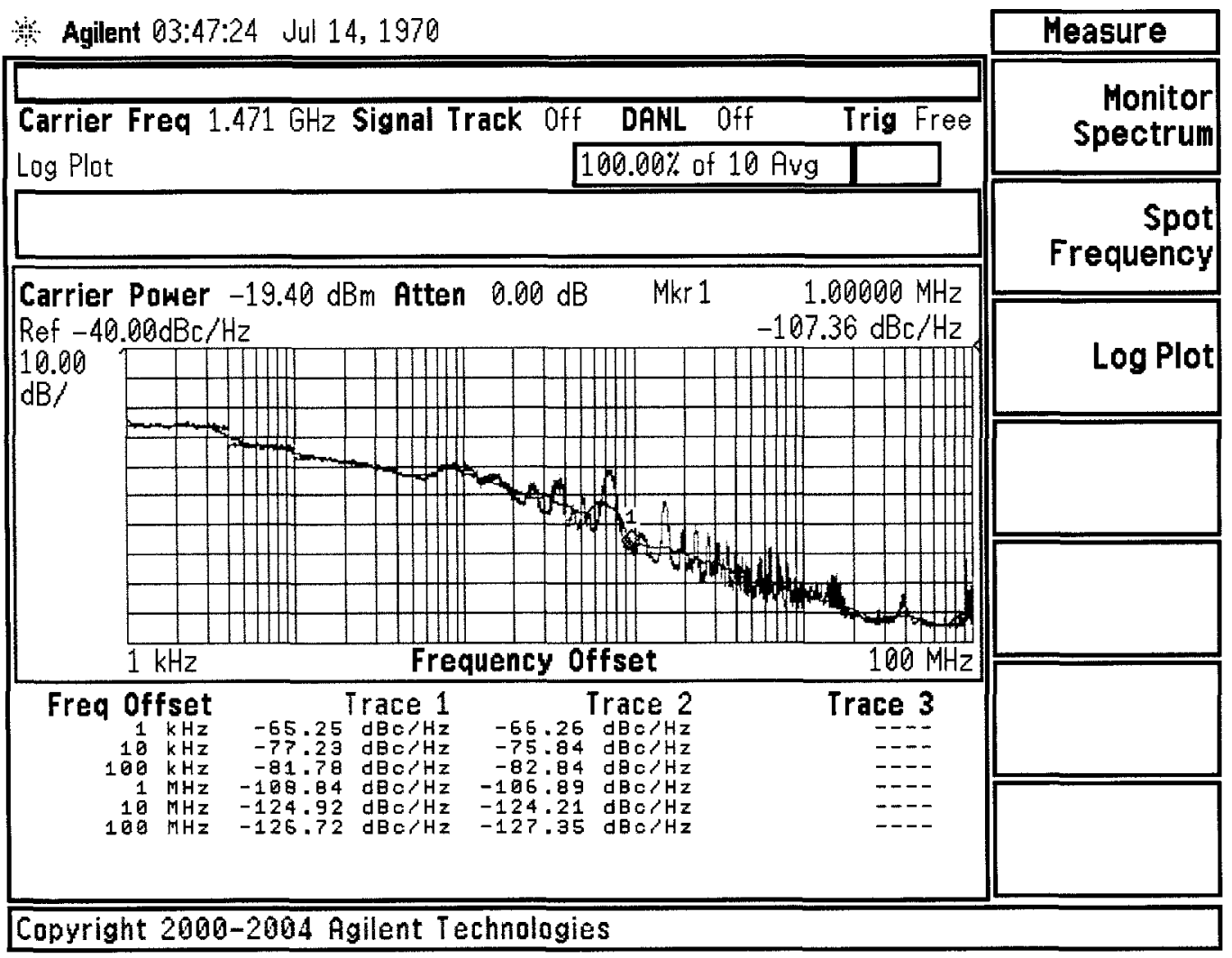

Figure 5.12: Plot of the output from the spectrum analyzer for the VCO without the switched-bias circuit, showing the phase noise when the VCO is centered at $1.47 \mathrm{GHz}$. 

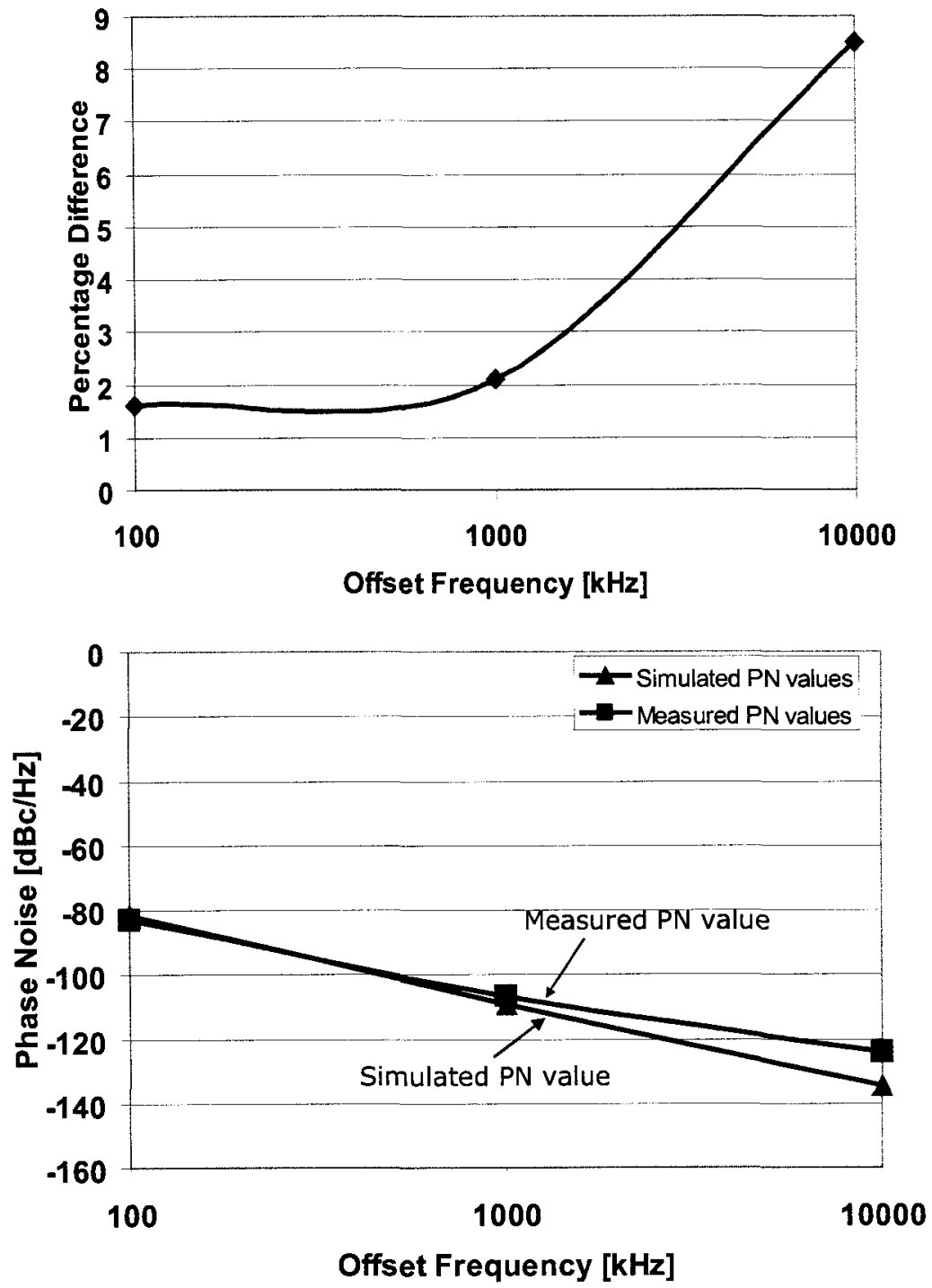

Figure 5.13: A comparison of the simulated and measured phase noise (PN) values for the VCO without the switched-bias circuitry is shown on the bottom curve. The top curve is the percentage difference between the simulated and measured VCO phase noise values. 
Using the frequencies listed in Table 5.4, the tuning range, $\mathrm{TR}_{V C O}$, was calculated, as a percentage, using equation,

$$
T R_{V C O}=\frac{f_{\text {high }}-f_{\text {low }}}{f_{\text {center }}} \times 100 \%
$$

where $\mathrm{f}_{\text {high }}$ is the highest VCO frequency, associated with the lowest control voltage, $\mathrm{Vctrl}=1.2 \mathrm{~V}$ for this $\mathrm{VCO}, \mathrm{f}_{\text {low }}$ is the lowest $\mathrm{VCO}$ frequency, associated with the highest control voltage, Vctrl $=1.8 \mathrm{~V}$ for this $\mathrm{VCO}$, and $\mathrm{f}_{\text {center }}$ is the VCO's center frequency, associated with the mid control voltage, which is $\mathrm{Vctrl}=1.5 \mathrm{~V}$ for this VCO.

Table 5.4: Frequency values used to determine the tuning range of the VCO

\begin{tabular}{|l||c|c|}
\hline Control voltage, Vctrl & Simulated Frequency & Measured Frequency \\
\hline $1.2 \mathrm{~V}$ & $1.51 \mathrm{GHz}$ & $1.49 \mathrm{GHz}$ \\
\hline $1.5 \mathrm{~V}$ & $1.41 \mathrm{GHz}$ & $1.47 \mathrm{GHz}$ \\
\hline $1.8 \mathrm{~V}$ & $1.27 \mathrm{GHz}$ & $1.49 \mathrm{GHz}$ \\
\hline
\end{tabular}

The simulated frequency values were measured by running a transient simulation using the schematic shown in section 4.1 and varying the control voltage, $V$ ctrl between $1.2 \mathrm{~V}$ and $1.8 \mathrm{~V}$, as was specified in [8]. The frequency was measured at the output of the VCO (Vout+ in this case, though Vout- showed an equal frequency). For the measured values, the test equipment was setup as shown in Figure 5.10 and the frequency was read from the spectrum analyzer as the control voltage, Vctrl, was swept from $1.2 \mathrm{~V}$ to $1.8 \mathrm{~V}$.

The frequency values match well with a percentage difference of $4.3 \%$ between the simulated and measured values. The power dissipation is $33 \%$ higher than expected. This is due to the fact that extra current was required to properly bias the VCO, indicating that the current mirror was not perfectly mirroring the current. The tuning range is much lower than expected, with a difference of $72 \%$ between the simulated and measured values. The cause of this is likely in part due to the modelling of the MOSCAPs done through the simulations; it appears that the simulations did not accurately predict the available range the MOSCAPs can achieve. It is possible that through the fabrication of the MOSCAPs the capacitance range expected changed, for example due to parasitic capacitance, reducing the tuning range available. 


\subsubsection{Testing of the VCO with the switched-bias circuit}

Through observations it was observed that the switches used in the switched-bias method are functional. A diagram of the test setup used to take the measurements is shown in Figure 5.14.

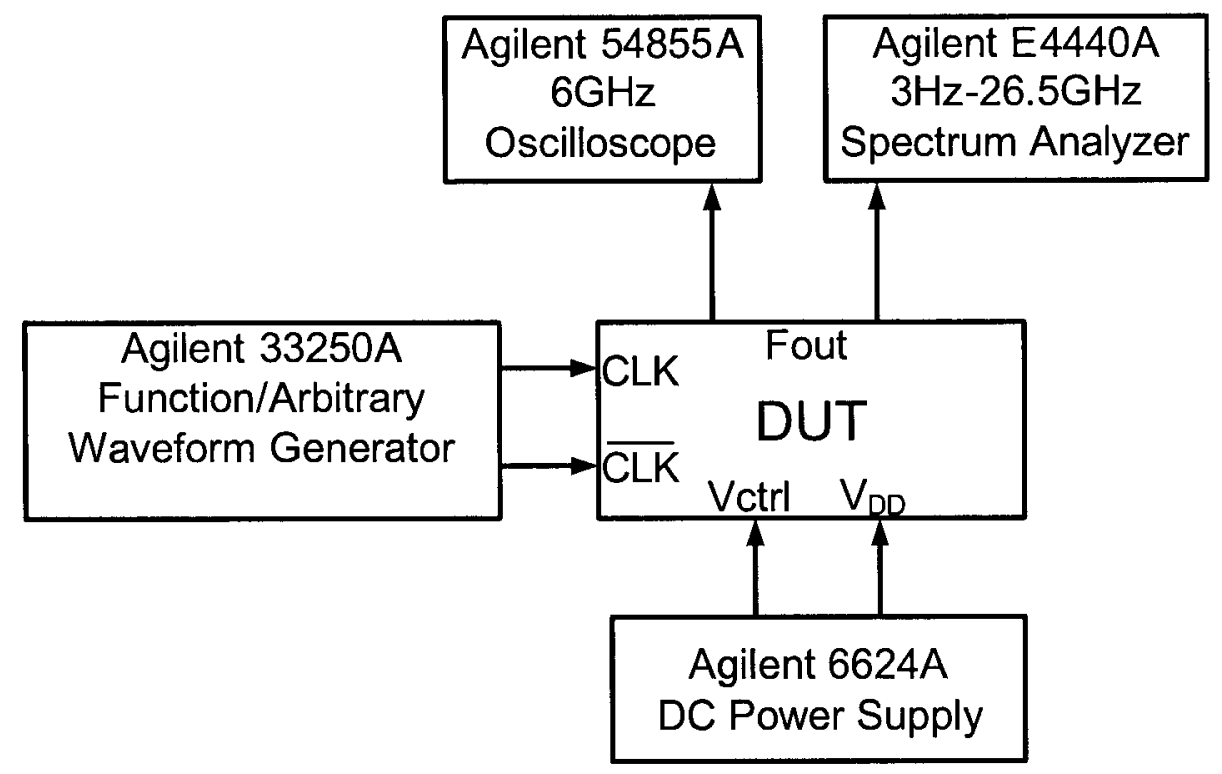

Figure 5.14: Test setup for the measurements taken for the VCO with the switched-bias circuitry.

The voltage variation, $\Delta \mathrm{V}_{B}$, at the bias node is a problem encountered in this circuit when the main switch is opened. The problem is illustrated in Figure 5.15. In this plot, a clock of $1 \mathrm{MHz}$ is being used to turn the switch on and off. When the switch is on, the voltage at the bias node remains at a constant value. When the switch is opened, the voltage at the bias node should continue to remain constant. However, due to leakage of the charge to the substrate, the voltage at the bias node begins to decrease. To further investigate this leakage, the voltage variation at the bias node, $\Delta V_{B}$, for clock frequencies between $100 \mathrm{kHz}$ to $10 \mathrm{MHz}$, in steps of $1 \mathrm{MHz}$ were examined. The results are shown in Figure 5.16. As expected, as the clock frequency increases the voltage variation at the bias node decreases because the capacitor has less time to discharge before the switch closes to begin charging the capacitor again. 


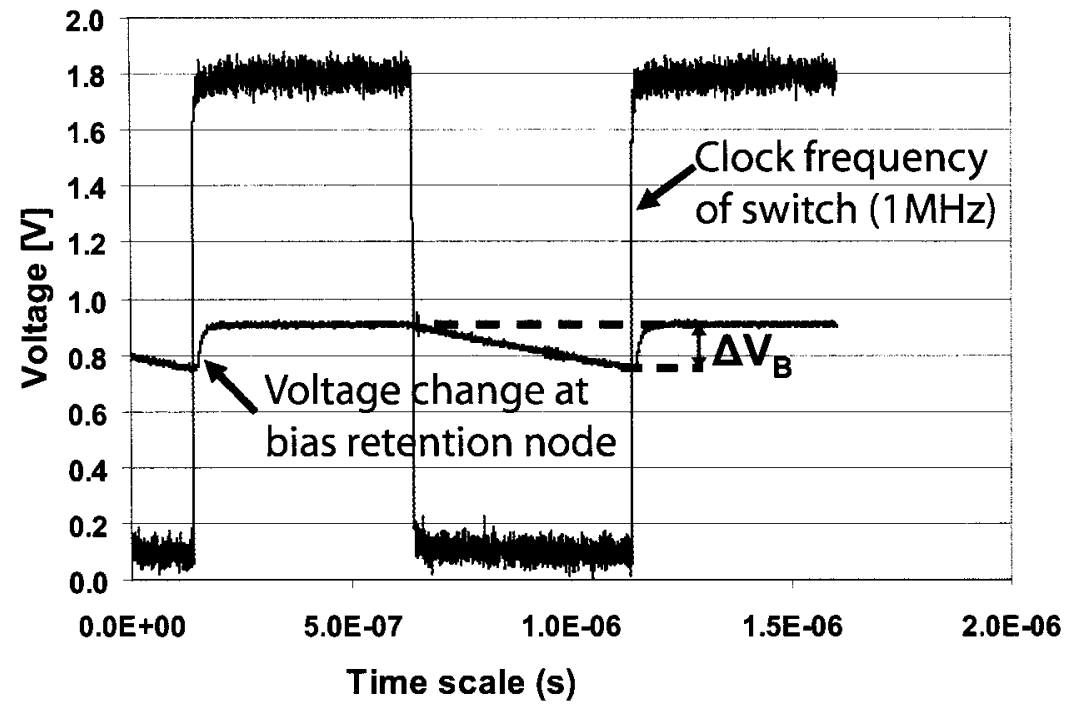

Figure 5.15: Plot showing the voltage change at the bias retention element, given by $\Delta \mathrm{V}_{B}$. The switch in the switched-bias circuit is being switched at $1 \mathrm{MHz}$.

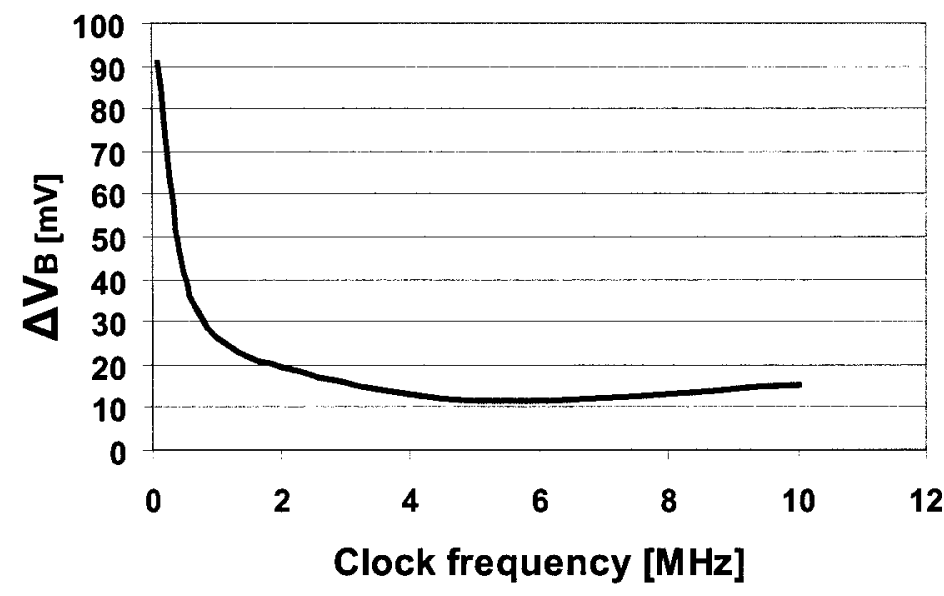

Figure 5.16: Plot showing the trend of the voltage variation, $\Delta \mathrm{V}_{B}$, at the capacitor, $\mathrm{C} 2$, as the clock frequency on the switch is changed. 


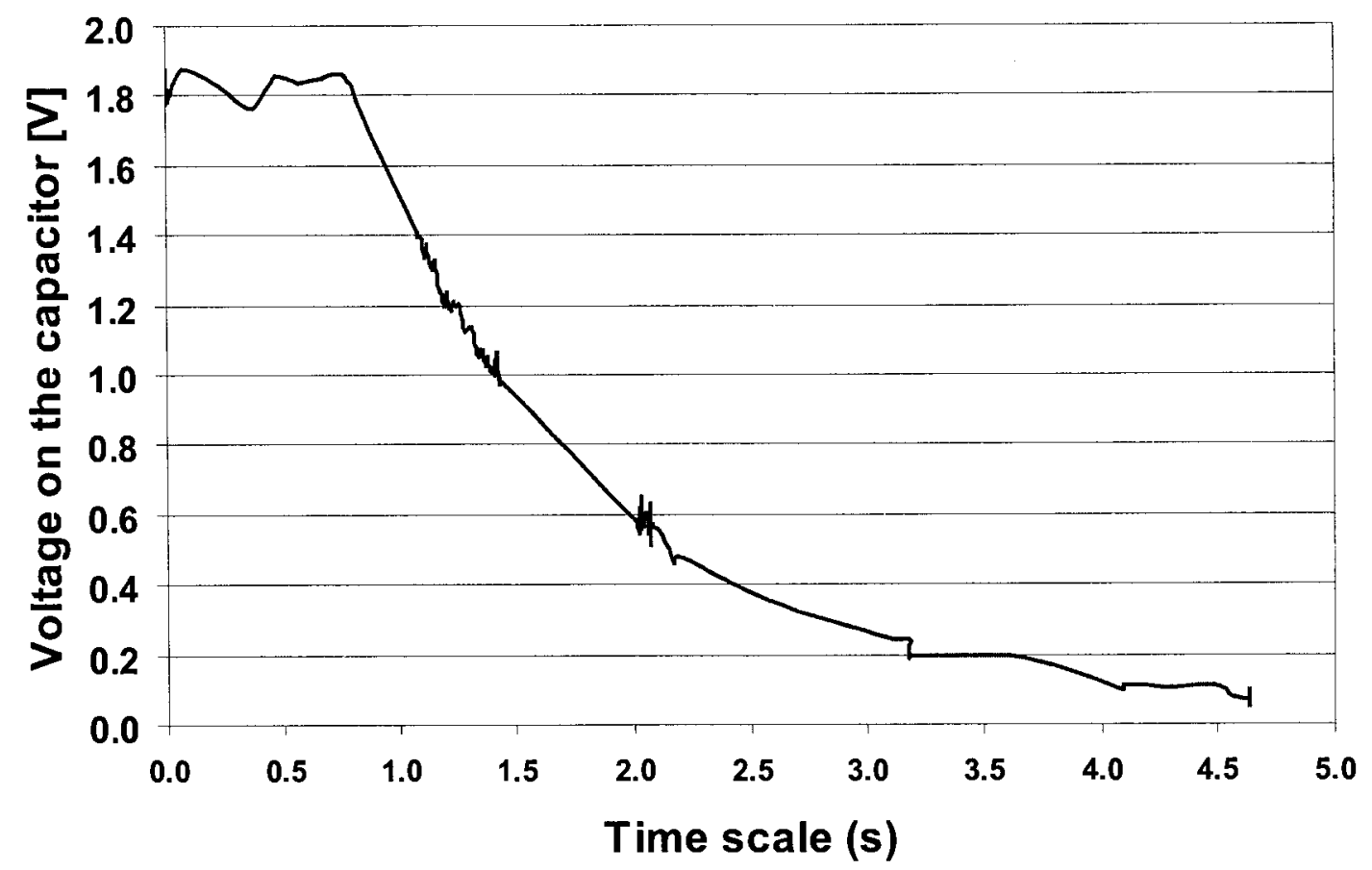

Figure 5.17: Plot showing the voltage variation observed due to the oscilloscope probes used to take measurements of the circuit. 
To ensure that the leakage value being measured was due to the circuit itself, and not due to the oscillator probe, the leakage in the probes was measured. This measurement was done using a capacitor isolated on an insulated surface. The capacitor ( $10 \mu \mathrm{F}$ surface mount capacitor) was charged to $1.8 \mathrm{~V}$; then the oscillator probes were attached to the capacitor and the change in voltage was monitored. As is shown in Figure 5.17 the leakage from the probes is minimal as it takes 3 seconds to discharge the capacitor. This result indicates that the leakage observed at the bias node of the circuit obtained during the measurements is mainly due to leakage of the charge through the substrate and not due to the oscilloscope probes.

\subsubsection{Challenges encountered during the testing of the VCO with the switched-bias circuit}

Though the VCO used to verify the switched-bias circuitry had been tested on its own (i.e. without the switched-bias circuit), when the VCO with the switched-bias circuit was tested it would not oscillate. Several tests were done to try and determine the reason for the lack of oscillations, and they will be discussed within this section. Seven chips were tested in all during this process.

To begin debugging the circuit, the bias currents were adjusted. Various combinations of increasing and decreasing the currents was tried. While the currents were being changed, the overall current was monitored to ensure the correct operation of the current mirror and that the overall current was within the expected range (of approximately $20 \mathrm{~mA}$, for the complete circuit, including the buffers that are used at the output of the design, as mentioned in section 4.1). These tests showed that the current mirrors were functioning properly, but that changing the biasing did not result in oscillations for the VCO.

The eliminate the possibility that the additional switched-bias circuitry was causing the problem, the VCO was biased directly through the VcapTest point, which was described in 5.1. VcapTest, which is located at the gate of M7 (refer to Figure 3.14), was included both as a measurement test point, as well as to allow external biasing the VCO, 
if required. However, externally biasing the VCO did not solve the problem, and the VCO continued to not have any oscillations.

No kick-start input had been included with the circuit due to the strength of the oscillations of the previous fabricated VCO, which also did not include a kick-start input. As space was at a premium on the chip, it was required to eliminate all but the essential circuitry. As a result there was external circuitry that could be used to manually start the oscillations. However, a pulse was placed at VDD to try and provide a kick-start to the VCO, but this technique did not result in oscillations at the output of the VCO.

However, testing continued to try and locate the cause of the problem. The layout was thoroughly inspected, and simulations were re-run to ensure that the fully extracted VCO worked properly. Before the chip was fabricated a complete LVS (i.e. including the pads) had been done to ensure all of the connections were made correctly. Therefore from a pre-fabrication (i.e. layout and schematic) point of view there was no reason found that would explain the lack of oscillations.

To determine if the buffers were possibly dampening the oscillations before they reached the output, a square wave signal was inputted at the input pad for Vctrl (the voltage control signal, which controls the MOSCAPs used for tuning), on top of the usual DC Vctrl signal (which was set to $1.5 \mathrm{~V}$ ). The goal of this test was to determine if the signal could travel from the VCO, through the buffers, and appear at the output. The frequency of the input was increased by $100 \mathrm{MHz}$ steps to determine if the frequency of the input had an effect on the waveform observed at the output. The output was observed both on the oscilloscope and the spectrum analyzer. The peak-to-peak amplitude voltage value was measured from the oscilloscope and is plotted in Figure 5.18.

The results showed that the signal did successfully travel through the on-chip buffers. The buffers attenuated the signal, as it is seen that the $500 \mathrm{mV}$ peak-to-peak input signal has an output peak-to-peak amplitude between $7 \mathrm{mV}$ and $21 \mathrm{mV}$. As expected there is generally greater attenuation of the signal (i.e. smaller amplitude) at frequencies away from the center frequency of $1.4 \mathrm{GHz}$; however a noticeable spike in amplitude was also noticeable at $900 \mathrm{MHz}$, which will be investigated in the next test discussed. This test of 


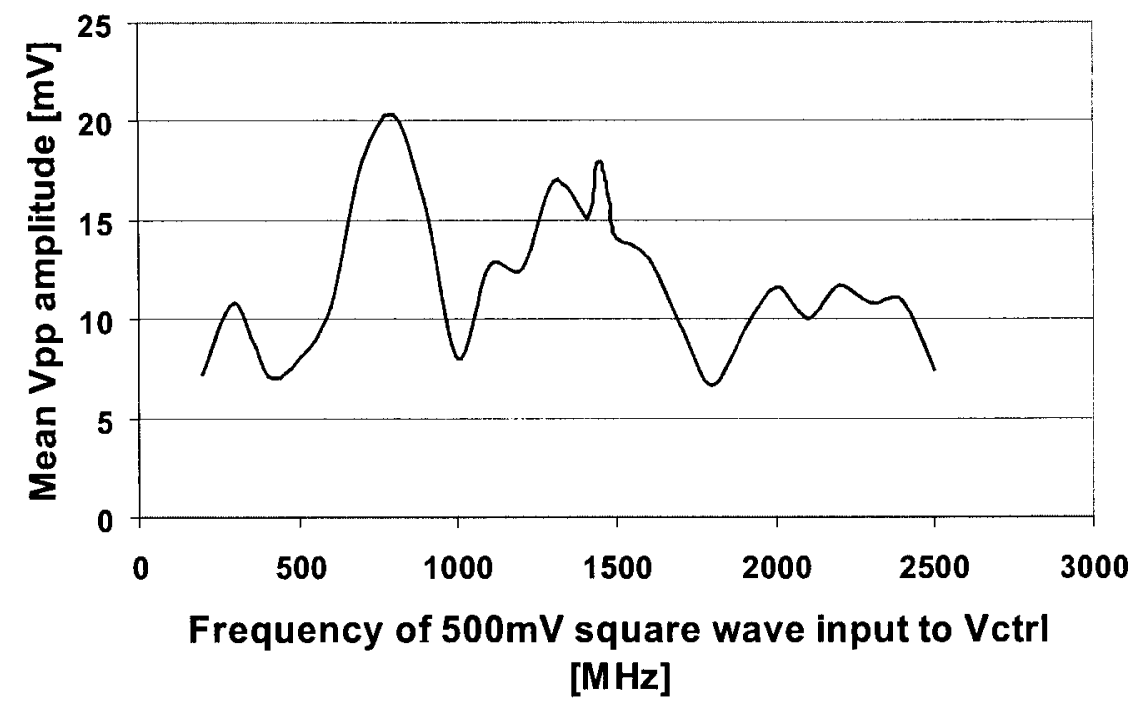

Figure 5.18: Measurement results showing the test used to verify the functionality of the buffers in the VCO. A square wave signal was inputted into Vctrl to determine if the signal could successfully travel through the buffers to the output.

the buffers proved that once the signal was present, it was able to appear at the output of the VCO, verifying that the buffers are not dampening the signal so much that that the result was a lack of oscillations at the output pad of the VCO on the PCB.

As mentioned above, from the buffer test measurements it was observed that, while there is the expected peak in amplitude near the desired frequency of $1.4 \mathrm{GHz}$, there is also another peak around $900 \mathrm{MHz}$. It was hypothesis that perhaps the bond wires were causing a secondary frequency to be present which was dominating the desired output frequency of $1.4 \mathrm{GHz}$. To test this hypothesize, simulations were done by varying the bond wire lengths to determine if this effect could be reproduced. The focus on this simulation was determining the effect of changing the bond wire length at $\mathrm{V}_{D D}$, and the results are shown in Figure 5.20 while the testbench used during the parametric sweep is shown in Figure 5.19. The bond wire was modelled using the "bondwire" model provided by CMC in the "package" library of Cadence. 500fF capacitors were used to model the pad capacitance on either side of the bond wire. The bond wire length was swept from $1 \mathrm{~mm}$ to $10 \mathrm{~mm}$ in $0.1 \mathrm{~mm}$ steps, as the frequency at the output was measured. The change in 


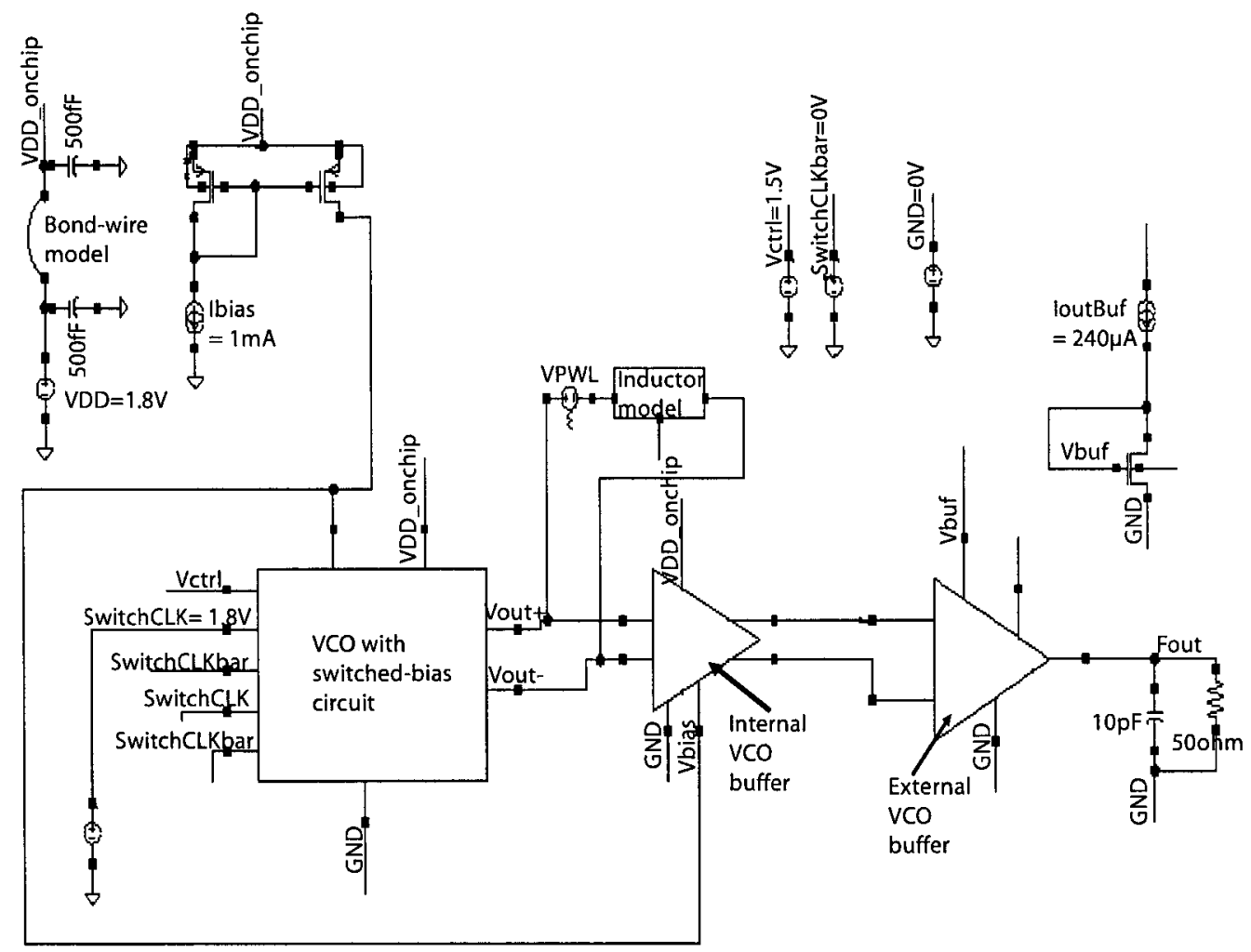

Figure 5.19: Testbench used to run the bond wire length parametric sweep simulation. 


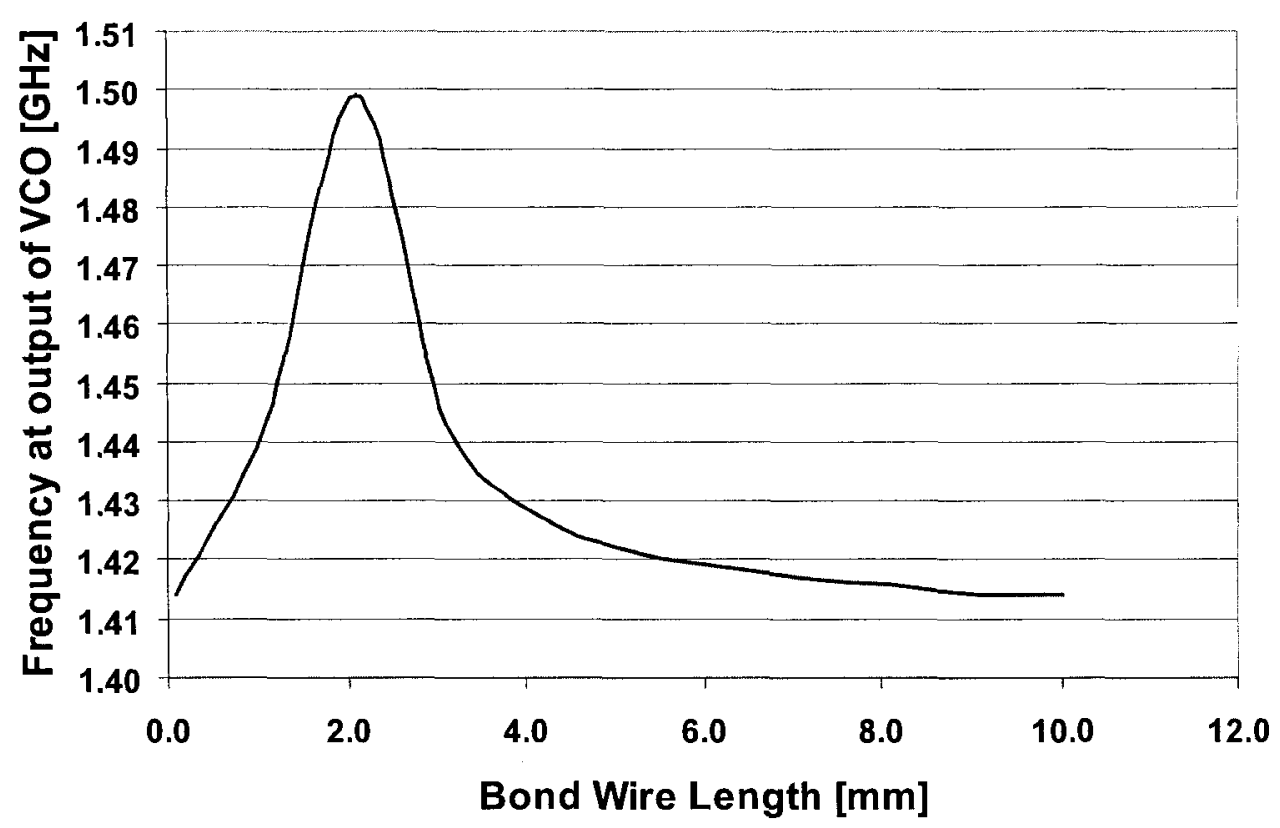

Figure 5.20: Simulation results showing the change in output frequency of the VCO as the bond wire length at $\mathrm{V}_{D D}$, the power supply, is changed.

bond wire length did have an impact on the output frequency, as is shown in Figure 5.20. The frequency increases to $1.5 \mathrm{GHz}$, indicating that the resonant frequency is changed by the length of bond-wire used due to extra inductance and capacitance from the bondwire. However, after approximately $4 \mathrm{~mm}$, the output frequency of the VCO returns to the $1.4 \mathrm{GHz}$ range, indicating that the $\mathrm{VCO}$ is no longer at the self-resonant frequency of the parasitic capacitance and inductance from the bond-wires. Overall the frequency value varied by $7 \%$, but remained within the expected range of $1.4 \mathrm{GHz}$ to $1.5 \mathrm{GHz}$ and did not have an additional frequency at $900 \mathrm{MHz}$, as was seen in the buffer testing measurement results. The likely reason for the extra frequency at $900 \mathrm{MHz}$ is noise in the testing environment which resulted in interference with the measurement result. Alternatively there is the potential there may have been extra harmonics that appeared at the output of the VCO during the buffer testing measurements.

To continue investigating reasons for the lack of oscillations for the VCO it was decided to simulate the VCO over various temperatures. The reasoning behind this was that it 


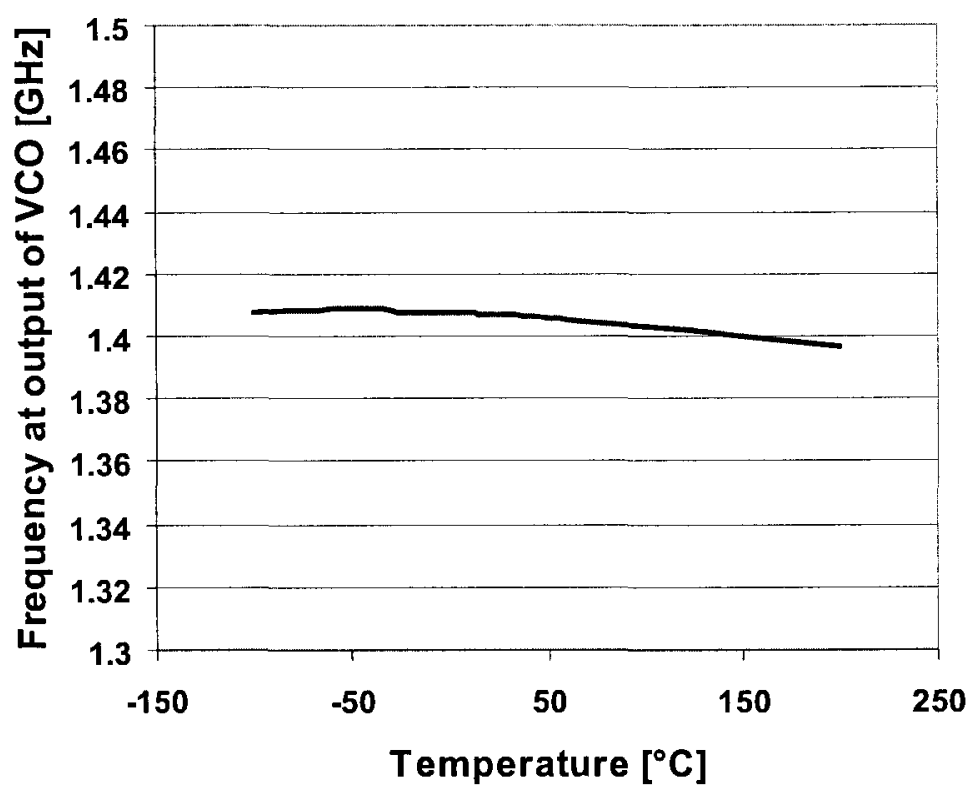

Figure 5.21: Simulation results showing the change in output frequency of the VCO as simulation temperature is swept.

is possible that the chip was heating up during its operation leading to incorrect output results (i.e. lack of oscillations). The testbench used for the temperature test is equivalent to the testbench shown in Figure 5.19, minus the bond-wires. The temperature in the simulator was varied and transient simulations were run to observe the frequency at the output of the VCO. As is shown in Figure 5.21, the VCO oscillates at the expected frequency of $1.4 \mathrm{GHz}$ over the range of temperatures simulations, from -100 degrees Celsius to 200 degrees Celsius. Therefore, the simulations show that temperature does not affect the frequency of oscillation of the VCO.

The conclusion after testing six fabricated chips is that the non-functionality of the chip appears to result from a problem with the fabrication of the core of the VCO itself. This conclusion is based on the fact that tests have indicated that the current mirrors are properly supplying the desired amount of current to the chip and that the buffers are functional, as has been demonstrated in this section. The possibilities for a lack of oscillations due to the core of the VCO itself could be from mismatch within the 
transistors, or potentially a problem with the tank, made up of the spiral inductor, the fixed capacitance and the MOSCAPs. A $10 \%$ mismatch of the transistor sizes was tried in simulations, but this test indicated that even with this large a mismatch the VCO would still continue to oscillate. Therefore, the most likely cause of the problem is the tank, especially as it was not possible to simulate the design with the extracted inductor, due to the problem of running an LVS with inductors in the circuit (i.e. the inductors appear as a short circuit). It is possible that, though the inductor is properly connected in the layout, there might have been a fabrication problem that resulted in an incorrect inductor value.

The lack of oscillations in the VCO have resulted in an inability to verify the switchedbias circuitry through measurements, though the simulation results show that this method appears to be promising in helping to reduce phase noise in VCOs.

\subsection{Chapter Summary}

- This chapter discuses the test setup and measurement results obtained from the fabricated chip. It begins with a look at the printed circuit board (PCB) design.

- The VCO without the switched-bias circuit was tested, and served as a benchmark against which to compare the VCO with the switched-bias circuit.

- It was observed that the chip with the modified VCO returned from fabrication did not oscillate. The steps used to debug the chip were discussed.

- Due to the lack of oscillations in the VCO application, the effectiveness of the switched-bias circuit could not be measured, however, the simulations that were shown in section 4.5 indicate that this technique appears to be effective in reducing phase noise in the simulated VCO. 


\section{Chapter 6}

\section{Conclusion}

\subsection{Summary of the results obtained}

Simulations have shown that for the switched-bias circuit used within the particular LC oscillator simulated there is a $6 \mathrm{~dB}$ drop observed in the VCO phase noise between the case when the switch is closed and opened. It was not possible to obtain measurement results from the fabricated chip to verify the decrease in phase noise, due to a lack of oscillations in the fabricated VCO.

However, the combination of the transfer function derived in subsection 3.4.2, coupled with the results from the noise simulation in subsection 3.4 .3 which showed that the transistors in the bias network contribute the largest amount of noise within the circuit, provide information supporting the validity of this technique. In addition, it has been shown that the addition of the switched-bias circuit did not negatively impact the bias network, as the current was still properly mirrored within the fabricated chip.

\subsection{Contribution to Research}

The switched-bias technique provides another means to reduce phase noise within a VCO. The switched-bias circuit is formed from an NMOS circuit and a MIM capacitor. This circuitry was placed within an LC oscillator to reduce that phase noise seen at the output of the VCO. The design and integration of the switched-bias circuit was developed. This included measuring the voltage variation that could be tolerated within the bias network. 
A transfer function was developed to show how the noise is transferred across the current mirror, and into the VCO. Simulations verified the transfer function, and simulations were further used to observe the change in VCO phase noise between the case when there was no switched-bias circuit in the VCO, when the main switch was kept closed, and when the main switch was opened.

The work described in this thesis was presented at the Wireless 2005 conference in Calgary, Alberta in July 2005 in a paper entitled "A Switched-Bias VCO Phase Noise Reduction Technique", which was co-authored by Céline Fletcher, Daniel Olszewski, Dr. Leonard MacEachern and Dr. Samy Mahmoud.

\subsection{Future Work}

There are several areas for future work related to the work presented in this thesis. The main area of interest would be in re-fabricating the chip to obtain a working VCO that could be used to verify the simulations obtained.

Different VCO configurations could be designed and tested with the switched-bias technique to see if a similar improvement is obtained or if the improvement is limited to LC oscillators. For example, it would be of interest to see if ring oscillators would be able to benefit from this technique as they generally have higher phase noise values than LC oscillators.

The switched-bias technique could also be expanded beyond bias network, and could, in theory, be used to segment large circuits into small components that are selectively turned on and off as required. This has the potential to allow a large circuit to save power as sections of the circuit are disconnected.

Variations of the circuit can be considered. The bias retention element, which was implemented using a MIM capacitor in this research, could be replaced with a MOS-capacitor to help save on area, as MOS capacitors are generally smaller than MIM capacitors. As well, the NMOS switch could be replaced with a lower leakage switch, which would enable the noise reduction experience to be extended for longer periods of time.

Finally, the effect of spurs that may occur due to the clock input to the switch need to 
be investigated. Filtering may be required to reduce spurs that occur close to the center frequency of the VCO. Tests would be required to determine if these spurs reduce the usefulness of the switched-bias technique within a VCO. 


\section{Appendix A}

\section{PCB Components}

The components shown in Table A.1 were used to populate the PCB used to test the fabricated chip.

Table A.1: Components used to populate the PCB that was described in section 5.1 and shown in Figure 5.4.

\begin{tabular}{|l||c|c|c|c|}
\hline Component & Size & Quantity & Manufacturer & Part Number \\
\hline Capacitor & $10 \mu \mathrm{F}$ & 6 & Kemet & T491B106K010AS \\
\hline Capacitor & $0.1 \mu \mathrm{F}$ & 8 & MuRata & GRM188R71C104KA01D \\
\hline Capacitor & $0.01 \mu \mathrm{F}$ & 3 & MuRata & GRM188R71H103KA01D \\
\hline Capacitor & $51 \mathrm{pF}$ & 1 & MuRata & GRM1885C1H510JA01 \\
\hline Capacitor & $0.5 \mathrm{pF}$ & 1 & MuRata & GRM1885C1HR50CZ01 \\
\hline Ferrite bead & $1000 \Omega$ & 3 & MuRata & BLM21AG102SN1D \\
\hline Potentiometer & $1 \mathrm{k} \Omega$ & 1 & Bourns & 3269-W-1-102 \\
\hline Potentiometer & $100 \Omega$ & 1 & Bourns & $3269-W-1-101$ \\
\hline Resistor & $0.1 \Omega$ & 4 & Panasonic-ECG & ERJ-3RSFR10V \\
\hline Zener Diode & N/A & 1 & Maxim-Dallas & DS9502P \\
\hline ESD protection & N/A & 3 & ON Semi & NUP4301MR6T1 \\
\hline SMA connector & N/A & 5 & Johnson & 142-0701-201 \\
\hline Current Source & N/A & 2 & National Semi & LM334Z \\
\hline
\end{tabular}




\section{Bibliography}

[1] T. Lee and A. Hajimiri, "Oscillator phase noise: A tutorial," IEEE Journal of SolidState Circuits, vol. 35, no. 3, pp. 326-336, March 2000.

[2] A. Hajimiri and T. Lee, "Design issues in CMOS differential LC oscillators," IEEE Journal of Solid-State Circuits, vol. 34, no. 5, pp. 717-724, May 1999.

[3] J. Phillips and K. Kundert, "Noise in mixers, oscillators, samplers, and logic an introduction to cyclostationary noise," in Proc. IEEE Custom Integrated Circuits Conference, May 2000, pp. 431-438.

[4] B. Razavi, "A study of phase noise in CMOS oscillators," IEEE Journal of Solid-State Circuits, vol. 31, no. 3, pp. 331-343, March 1996.

[5] A. Demir, A. Mehrotra, and J. Roychowdhury, "Phase noise in oscillators: A unifying theory and numerical methods for characterization," IEEE Transactions on Circuits and Systems - I: Fundamental Theory and Applications, vol. 47, no. 5, pp. 655-674, May 2000.

[6] J. Rael and A. Abidi, "Physical processes of phase noise in differential LC oscillators," in Proc. IEEE Custom Integrated Circuits Conference, May 2000, pp. 569-572.

[7] E. Hegazi, H. Sjöland, and A. Abidi, "A filtering technique to lower LC oscillator phase noise," IEEE Journal of Solid-State Circuits, vol. 36, no. 12, pp. 1921-1930, December 2001.

[8] D. Olszewski, "The design of a voltage controlled oscillator and charge pump for a radio over fibre receiver," 4th year project (Carleton University), 2003.

[9] D. Vye. (2004) Improving VC phase noise performance through enhanced characterization. 04.01HFE.pdf. [Online]. Available: http://www.ansoft.com/news/articles/

[10] O. Boric-Lubecke, J. Lin, and P. Gould, "Silicon chips for GSM base station receivers," 5th International Conference on Telecommunications in Modern Satellite, Cable and Broadcasting Services, TELSIKS 2001, vol. 2, pp. 381-388, September 2001. 
[11] J. Craninckx, M. Steyaert, and H. Miyakawa, "Fully integrated spiral-LC CMOS VCO set with prescaler for GSM and DCS-1800 systems," IEEE Proceedings of the Custom Integrated Circuits Conference, pp. 403 - 406, 1997.

[12] T. Liu, "6.5GHz monolithic CMOS voltage-controlled oscillator," Digest of Technical Papers - IEEE International Solid-State Circuits Conference, pp. 404-405, February 1999.

[13] C. Lam and B. Razavi, "2.6GHz/5.2GHz CMOS voltage-controlled oscillator," Digest of Technical Papers - IEEE International Solid-State Circuits Conference, pp. 402 403, February 1999.

[14] M. Do, R. Zhao, K. Yeo, and J. Ma, "New wideband/dualband CMOS LC voltagecontrolled oscillator," IEE Proceedings: Circuits, Devices and Systems, vol. 150, no. 5, pp. 453 - 459, October 2003.

[15] B. Park and P. Allen, "Low-power, low-phasc-noise CMOS voltage-controlledoscillator with integrated LC resonator," Proceedings - IEEE International Symposium on Circuits and Systems, vol. 4, pp. 421 - 424, May 1998.

[16] B. Razavi, "1.8 GHz CMOS voltage-controlled oscillator," Digest of Technical Papers - IEEE International Solid-State Circuits Conference, vol. 40, pp. 388 - 389, February 1997.

[17] B. Come, R. Ness, S. Donnay, L. V. der Perre, W. Eberle, P. Wambacq, M. Engels, and I. Bolsens, "Impact of front-end non-idealities on bit error rate performance of WLAN-OFDM transceivers," IEEE Radio and Wireless Conference (RAWCON 2000), pp. 91-94, September 2000.

[18] S. Levantino, C. Samori, A. Bonfanti, S. Gierkink, A. Lacaita, and V. Boccuzzi, "Frequency dependence on bias current in $5 \mathrm{GHz}$ CMOS VCOs: impact on tuning range and flicker noise upconversion," IEEE Journal of Solid-State Circuits, vol. 37, no. 8, pp. 1003-1011, August 2002.

[19] E. Klumperink, S. Gierkink, A. van der Wel, and B. Nauta, "Reducing MOSFET $1 / \mathrm{f}$ noise and power consumption by switched biasing," IEEE Journal of Solid-State Circuits, vol. 35, no. 7, pp. 994-1001, July 2000.

[20] J. Long, J. Foo, and R. Weber, "A $2.4 \mathrm{GHz}$ low-power low-phase-noise CMOS LC VCO," in IEEE Computer Society Annual Symposium on VLSI Emerging Trends in VLSI Systems Design (ISVLSI'04), February 2004, pp. 213-214.

[21] J. Chang, Y. Youn, M. Park, and C. Kim, "A new $6 \mathrm{GHz}$ fully integrated low power low phase noise CMOS LC quadrature VCO," in IEEE Radio Frequency Integrated Circuits Symposium, June 2003, pp. 295-298. 
[22] F. Svelto, S. Deantoni, and R. Castello, "A $1.3 \mathrm{GHz}$ low-phase noise fully tunable CMOS LC VCO," IEEE Journal of Solid-State Circuits, vol. 35, no. 3, pp. 356-361, March 2000.

[23] S. Wu, R. Liu, and W. Chen, "A 5.8-GHz high efficient, low power, low phase noise CMOS VCO for IEEE 802.11a," Proceedings of the 3rd IEEE International Workshop on System-on-Chip for Real-Time Applications, pp. 94-97, July 2003.

[24] M. Tiebout, "Low-power low-phase-noise differentially turned quadrature VCO design in standard CMOS," IEEE J.Solid-State Circuits, vol. 36, no. 7, pp. 1018-1024, July 2001.

[25] T. Ahrens, A. Hajimiri, and T. Lee, "A 1.6GHz 0.5mW CMOS LC low phase noise VCO using bond wire inductance," in Proc. 1997 Intl. Workshop on Design of MixedMode Integrated Circuits and Applications, pp. 69-71, July 1997.

[26] T. Ahrens and T. Lee, "A $1.4 \mathrm{GHz} 3 \mathrm{~mW}$ CMOS LC low phase noise VCO using tapped bond wire inductances," in Proc. 1998 Low Power Electronics and Design, pp. 16-19, August 1998.

[27] R. Dehghani and S. Atarodi, "Optimised analytic designed 2.5pGHz CMOS VCO," Elec. Letters, vol. 39, no. 16, pp. 1160-1162, August 2003.

[28] K. Takagi, "A study on phase-noise reduction method in phase-locked loop systems," IEEE Transactions on Ultrasonics, Ferroelectrics, and Frequency Control, vol. 50, no. 9, pp. 1207-1209, September 2003.

[29] S. Ye, L. Jansson, and I. Galton, "A multiple-crystal interface PLL with VCO realignment to reduce phase noise," IEEE Journal of Solid-State Circuits, vol. 37, no. 12, pp. 1795-1803, December 2002.

[30] K. Lundberg. (2002) Noise sources in bulk CMOS. CMOSnoise.pdf. [Online]. Available: http://web.mit.edu/klund/www/

[31] D. Johns and K. Martin, Analog Integrated Circuit Design. Canada: John Wiley and Sons, Inc., 1997.

[32] S. Magierowski, "Nonlinear noise analysis of LC-Tuned CMOS VCOs," Ph.D. dissertation, University of Toronto, Department of Electrical and Computer Engineering, 2004 .

[33] N. Gray, "Noise sources in bulk CMOS," On-line article, http://www.national.com/nationaledge/apr01/ab102.html, February 2005.

[34] N. Checka, D. Wentzloff, A. Chandrakasan, and R. Reif, "The effect of substrate noise on VCO performance," IEEE Radio Frequency Integrated Circuits RFIC Symposium, pp. 523-526, June 2005. 
[35] R. Baker, H. Li, and D. Boyce, CMOS: Circuit Design, Layout and Simulation. New York, NY: IEEE Press, 1998.

[36] J. Rogers and C. Plett, Radio Frequency Integrated Circuit Design. Boston, MA: Artech House, 2003.

[37] R. van de Plassche, J. Huijsing, and W. Sansen, Analog Circuit Design: RF Analogto-Digitals Converters; Sensor and Actuator Interfaces; Low-Noise Oscillators, PLLs and Synthesizers. Norwell, MA, U.S.A: Kluwer Acadmic Publishers, 1997.

[38] D. Steinback, "Oscillator phase noise reduction using nonlinear design techniques," Master's thesis, Virginia Polytechnic Institute and State University, October 2003.

[39] L. Wong, S. Hossain, and A. Walker, "Leakage current cancellation techniques for low power switched-capacitor circuits," in International Symposium on Low Power Electronics and Design, August 2001, pp. 310-315.

[40] D. Binkley, C. Hopper, J. Tucker, B. Moss, J. Rochelle, and D. Foty, "A CAD methodology for optimizing transistor current and sizing in analog CMOS design," IEEE Transactions Circuits and Systems - II, Analog Digital Signal Process, vol. 22, no. 2, pp. 225-237, February 2003.

[41] K. K. Hung, P. K. Ko, C. Hu, and Y. C. Cheng, "A unified model for the flicker noise in metal-oxide-semiconductor field-effect transistors," IEEE Transactions on Electron Devices, vol. 37, no. 3, pp. 654-665, March 1990.

[42] TSMC, "TSMC $0.18 \mu \mathrm{m}$ mixed signal 1P6M salicide $1.8 \mathrm{v} / 3.3 \mathrm{v}$ RF spice models," Technology Documentation, 2002.

[43] B. Razavi, RF Microelectronics. Upper Saddle River: Prentice Hall, Inc., 1998.

[44] Agilent ADS help manual. (2005) OscPort2 (differential oscillator port). ccsim045.html. [Online]. Available: ADS2004A/doc/ccsim/ccsim045.html

[45] M. Curtin and P. O'Brien, "Phase-locked loops for high-frequency receivers and transmitters," Analog Dialogue, pp. 1-5, 1999.

[46] B. Razavi, Design of Analog CMOS Integrated Circuits. Boston: McGraw-Hill, 2001.

[47] K. Kundert. (2005) Simulating switched-capacitor filters with spectrerf. The Designer's Guide Community. [Online]. Available: http://designers-guide.org

[48] T. Lee and A. Hajimiri, "A general theoryof phase noise in electrical oscillators," IEEE Journal of Solid-State Circuits, vol. 33, no. 2, pp. 179-194, February 1998.

[49] N. Gray. (2001) Application brief 102: Power supply effects on noise performance. AppBrief102.pdf. [Online]. Available: http://www.national.com/appbriefs/files/ 
[50] National Semiconductor. (2000) LM134/LM234/LM334 3-terminal adjustable current sources data sheet. LM334Z.html. [Online]. Available: http://www.alldatasheet.com/datasheet-pdf/view/NSC/

[51] ON Semiconductor. (2003) Low capacitance diode array for esd protection in four data lines. NUP4301MR6T1-D.PDF. [Online]. Available: http://www.onsemi.com/pub/Collateral/

[52] Dallas Semiconductor. (2003) Esd protection diode. DS9502.pdf. [Online]. Available: http://pdfserv.maxim-ic.com/en/ds/ 